\title{
THE LOCAL STRUCTURE THEOREM, THE NON-CHARACTERISTIC 2 CASE
}

\author{
CHRIS PARKER AND GERNOT STROTH
}

\begin{abstract}
Let $p$ be a prime, $G$ a finite $\mathcal{K}_{p}$-group, $S$ a Sylow $p$ subgroup of $G$ and $Q$ be a large subgroup of $G$ in $S$. The aim of the Local Structure Theorem [11] is to provide structural information about subgroups $L$ with $S \leq L, O_{p}(L) \neq 1$ and $L \not \leq N_{G}(Q)$. There is, however, one configuration where no structural information about $L$ can be given using the methods in 11. In this paper we show that for $p=2$ this hypothetical configuration cannot occur. We anticipate that our theorem will be used in the programme to revise the classification of the finite simple groups.
\end{abstract}

\section{INTRODUCTION}

The proof of the classification of the finite simple groups took different directions depending upon the structure of normalizers of nontrivial 2-subgroups. Such subgroups are called 2-local subgroups. If $M$ is such a 2-local subgroup, then there are two possibilities $C_{M}\left(O_{2}(M)\right) \leq$ $O_{2}(M)$ or $C_{M}\left(O_{2}(M)\right) \not \leq O_{2}(M)$. In the former case, we say that $M$ has characteristic 2 . If all the 2-local subgroups of a finite group $G$ have characteristic 2, then we say that $G$ is of local characteristic 2 . The classification divides into the investigation of groups which are of local characteristic 2 and those which are not. In the latter case the objective is to show that there is a 2-local subgroup which has a fairly simple structure (a subnormal $\mathrm{SL}_{2}(q)$, standard subgroups). One of the main obstructions for proving the existence of such a 2-local subgroup is the existence of non-trivial normal subgroups of odd order in 2-local subgroups. A new approach due to M. Aschbacher (for an overview see [1, Chapter 2]) using fusion systems avoids this problem. The first steps of this programme can be found in a preprint [2].

For groups of local characteristic 2, the problem is the complexity of the structure of the 2-local subgroups. In the original classification, to avoid this complexity problem the strategy was to move to $p$-local subgroups for suitable odd primes $p$, which then eventually have a fairly restricted structure similar to standard subgroups. 
In the years following the classification, methods for working with 2-local subgroups of groups of local characteristic 2 have been refined and developed. These new methods inspired a novel approach to the classification of groups of local characteristic 2 initiated by U. Meierfrankenfeld, B. Stellmacher and G. Stroth (see [10] for an overview), the MSS-programme for short, which stays in the 2-local world and intends to pin down the structure of $G$. The Local Structure Theorem [11] provides information about important subgroups and quotients of certain 2-local subgroups and further work is in progress. A tempting possibility is that there is a bridge between Aschbacher's programme and the MSS-programme which means they can be merged to give a new proof of the classification of the finite simple groups. One of the purposes of this paper is to build part of such a bridge.

We now explain how these two programmes can possibly be joined. For this we have to say a little bit more about Aschbacher's approach. As an example, let us assume that we have a 2-local subgroup $M \cong$ $2 \times \operatorname{Alt}(5)$. Then our target simple group is the sporadic simple group $\mathrm{J}_{1}$, but the groups $\mathrm{SL}_{2}(16): 2$ and Alt(5) 22 also have such a 2-local subgroup, of course they are not simple. However to detect this fact takes a lot of work. To avoid this problem Aschbacher assumes that $M$ contains an elementary abelian 2-subgroup of $G$ of maximal order. With this extra condition $\mathrm{J}_{1}$ is the unique solution (assuming $O_{2}(G)=O(G)=1$ ). The problem is that an approach to the classification based on Aschbacher's new work no longer has the tidy division into two cases: local characteristic 2 or not local characteristic 2 . To take the discussion further, we introduce the notion of parabolic characteristic 2 . This means that we require $M$ has characteristic 2 only for those 2-local subgroups $M$ of odd index in $G$. If we could classify the groups of parabolic characteristic 2, then this would be a counterpart to Aschbacher's work and together they would provide an alternative proof of the classification. At the moment providing such classification seems to be out of reach. However, it is also more than is required. Fix $S \in \operatorname{Syl}_{2}(G)$ and recall the Baumann subgroup of $S$ is defined to be $B(S)=C_{S}\left(\Omega_{1}(Z(J(S)))\right.$. For a saturated fusion system $\mathcal{F}$ on a 2-group $T$, Aschbacher considers components of $C_{\mathcal{F}}(t)$ where $t$ is an involution with $m_{2}(T)=m_{2}\left(C_{T}(t)\right)$ (see [2, page 5]). So, if Aschbacher's programme is successful, then we can assume that $C_{G}(t)$ has characteristic 2 for all involutions in $\Omega_{1}(Z(J(S)))$. Hence, if we could determine the groups in which every 2-local subgroup containing $B(S)$ has characteristic 2, then we could meld the two programme and produces an alternative proof of the classification. Such groups are called groups of Baumann characteristic 2. 
So far the investigation in MSS focuses on groups which possess a large subgroup $Q$ (the exact definition will be given later on). A consequence of the existence of such a group is that $G$ has parabolic characteristic 2. The Local Structure Theorem in [1] gives information about the structure of groups of parabolic characteristic 2, which have a large subgroup $Q$. In fact this has been done for arbitrary primes $p$. For a $p$-local subgroup $M$ of characteristic $p$, there is a unique nontrivial normal elementary abelian $p$-subgroup $Y_{M}$ maximal subject to $O_{p}\left(M / C_{M}\left(Y_{M}\right)\right)=1$. The Local Structure Theorem gives information about $Y_{M}$ and the action of $M / C_{M}\left(Y_{M}\right)$ on $Y_{M}$ provided $Q$ is not normal in $M$ and $M$ contains a Sylow $p$-subgroup of $G$. To take the investigation further there are two cases to be investigated. Either $Y_{M} \not \leq Q$ for some such $M$ or $Y_{M} \leq Q$ for all such $M$. In both instances define

$$
H=\left\langle K \mid O_{p}(K) \neq 1, S \leq K\right\rangle .
$$

In the first case, the $H$-Structure Theorem (work in preparation) builds on the Local Structure Theorem and determines the group $H$. Using this, for $p=2, F^{*}(G)$ can be identified. If $p$ is odd, then either $F^{*}(G)$ is determined, or $F^{*}(H)$ is demonstrated to be a group of Lie type in characteristic $p$ and rank at least three, or $H$ is a weak $B N$-pair. Up to this point the MSS-programme fits well with Aschbacher's point of view. In the second case, $Y_{M} \leq Q$ for all $M$, and again we intend to determine the group $H$. For this, the first question is: which of the $p$ local subgroups from the Local Structure Theorem can show up? This has been partly answered in [9, 12] but only under the assumption that $G$ has local characteristic $p$ and this assumption is not compatible with Aschbacher's approach. Hence we must replace it by a more applicable premise. The starting point for [9, 12] is [11, Corollary B] which lists the cases from the Local Structure Theorem which may appear when $Y_{M} \leq Q$ and $G$ is of local characteristic $p$. Using this information [9, 12] basically exclude what is called the wreath product case in the Local Structure Theorem. From now on assume that $p=2$ as this is the relevant prime for Aschbacher's approach. The first question is: what happens if we remove the assumption of local characteristic 2 in [11, Corollary B]? The answer is that two further configurations for the group $M$ appear. One is that $M / C_{M}\left(Y_{M}\right)$ induces the natural $\mathrm{O}_{2 n}^{ \pm}(2)$ module on $\left[Y_{M}, M\right]$. This possibility has been handled by Chr. Pröseler [15] in his PhD thesis. The second is that [11, Theorem A (10-1)] holds and this is the situation handled in this paper. We will show that no groups satisfy this hypothesis. Hence we may investigate the proofs of [9, 12] starting with the same set of possible 2-local subgroups as 
provided by [11, Corollary B]. However the proofs in [9, 12] also exploit that $G$ has local characteristic 2 but only for 2-local subgroups $K$ which contain the Baumann subgroup $B(S)$. Hence the local characteristic 2 assumption can be replaced with Baumann characteristic 2 and we then obtain the same conclusion as in [9, 12, Therefore, provided we can prove the analogue of the $H$-structure theorem in the case that $Y_{M} \leq Q$ for all $M$ when $G$ has Baumann characteristic 2, we will have a companion to Aschbacher's approach.

To explain further the context of the results in this article, we give a simplified overview of the Local Structure Theorem for the particular case when $p=2$ and then outline the contribution of the research in this paper. We work in an environment compatible with being a counter example to the classification. Thus we call $G$ a $\mathcal{K}_{2}$-group if and only if every simple section of every 2-local subgroup of $G$ is in the set of known simple groups $\mathcal{K}$ where $\mathcal{K}$ consists of the groups of prime order, the alternating groups, the simple groups of Lie type and the sporadic simple groups.

A subgroup $Q$ of $S$ is called large if

- $Q=O_{2}\left(N_{G}(Q)\right)$;

- $C_{G}(Q) \leq Q$; and

- for any $1 \neq A \leq Z(Q)$ we have that $Q$ is normal in $N_{G}(A)$.

For $K \leq H \leq G$ we define

$$
\mathcal{L}_{H}(K)=\left\{K \leq L \leq H \mid O_{2}(L) \neq 1, C_{H}\left(O_{2}(L)\right) \leq O_{2}(L)\right\} .
$$

As we asserted earlier, the existence of the large subgroup $Q$ implies that $G$ has parabolic characteristic 2 and so, in this case, $\mathcal{L}_{G}(S)$ contains all of the 2-local subgroups of $G$ which contain $S$. Define $\mathfrak{M}_{G}(S)$ to be the subset of $\mathcal{L}_{G}(S)$ which contains the subgroups $M \in \mathcal{L}_{G}(S)$ such that, setting $M^{\dagger}=M C_{G}\left(Y_{M}\right)$,

- $\mathcal{L}_{G}(M)=\mathcal{L}_{M^{\dagger}}(M)$ and $Y_{M}=Y_{M^{\dagger}}$; and

- $C_{M}\left(Y_{M}\right) / O_{2}(M) \leq \Phi\left(M / O_{2}(M)\right)$.

For $L \in \mathcal{L}_{G}(S)$ with $L \not \leq N_{G}(Q)$, define

$$
L^{\circ}=\left\langle Q^{L}\right\rangle \text {. }
$$

With these assumptions and notation the Local Structure Theorem states:

Suppose that $G$ is a $\mathcal{K}_{2}$-group, $S \in \operatorname{Syl}_{2}(G)$ and $S$ is contained in at least two maximal 2-local subgroups of $G$. Assume that $Q$ is a large subgroup of $G$ contained in $S$ and $M \in \mathfrak{M}_{G}(S)$ with $M \not \subset N_{G}(Q)$. Then the structure of $M^{\circ} / C_{M^{\circ}}\left(Y_{M}\right)$ and its action on $Y_{M}$ are described unless [11, Theorem A (10-1)] holds. 
So far so good, but what is this mysterious clause (10-1) in [11, Theorem A]? In this case, all that is proved is that $Y_{M}$ is tall and asymmetric in $G$, but importantly $Y_{M}$ is not characteristic $p$-tall in $G$. We will now explain in detail what this means, as our intention is to remove this restriction to the Local Structure Theorem for the situation when $p=2$ so that the we can provide the bridge to the Aschbacher project.

Let $M \in \mathfrak{M}_{G}(S)$ and $T \in \operatorname{Syl}_{2}\left(C_{G}\left(Y_{M}\right)\right)$. The subgroup $Y_{M}$ is

- tall, if there exists $K$ with $T \leq K \leq G$ such that $O_{2}(K) \neq 1$ and $Y_{M} \nless O_{2}(K)$, and

- asymmetric in $G$, if whenever $g \in G$ and $\left[Y_{M}, Y_{M}^{g}\right] \leq Y_{M} \cap Y_{M}^{g}$, then $\left[Y_{M}, Y_{M}^{g}\right]=1$.

Further $Y_{M}$ is characteristic 2-tall provided

- there is some $K$ with $T \leq K \leq G$ such that $C_{K}\left(O_{2}(K)\right) \leq$ $\mathrm{O}_{2}(K)$ and $Y_{M} \not \leq O_{2}(K)$.

We can now state the theorem we shall prove in this paper

Theorem. Let $G$ be a finite $\mathcal{K}_{2}$-group and $S \in \operatorname{Syl}_{2}(G)$. Suppose that $S$ is contained in at least two maximal 2-local subgroups and that $Q$ is a large subgroup of $G$ in $S$. Assume that there exists $M \in \mathfrak{M}_{G}(S)$ such that $Y_{M}$ is asymmetric and tall. Then $Y_{M}$ is characteristic 2-tall.

This theorem shows that for $p=2$ [11, Theorem A (10-1)] does not arise for $M \in \mathfrak{M}_{G}(S)$ and so also implies that Theorem A (10-1) of the Local Structure Theorem does not occur for arbitrary $L \in \mathcal{L}_{G}(S)$ with $L \not \subset N_{G}(Q)$ as [11, Theorem A (10-1)] states that there exists $M \in \mathfrak{M}_{G}(S)$ with $Y_{L}=Y_{M}$ and $L^{\circ}=M^{\circ}$.

For the proof of the theorem of this paper we assume that there exists $M \in \mathfrak{M}_{G}(S)$ with $Y_{M}$ asymmetric and tall, but not characteristic 2-tall. This means that for $T \in \operatorname{Syl}_{2}\left(C_{M}\left(Y_{M}\right)\right)$ if $K$ is a subgroup of $G$ containing $T$ with $O_{2}(K) \neq 1$ and $Y_{M} \not \leq O_{2}(K)$, then $C_{G}\left(O_{2}(K)\right) \not \leq$ $\mathrm{O}_{2}(K)$. Thus there are involutions $y \in Y_{M}$ such that $C_{G}\left(O_{2}\left(C_{G}(y)\right)\right) \not$ $\mathrm{O}_{2}\left(C_{G}(y)\right)$. We study these centralizers and would like to show that $E\left(C_{G}(y)\right) \neq 1$. That is, the centralizers have components. The obstruction to this is the existence of normal subgroups of odd order. The key for removing this obstacle is that $C_{G}(y)$ contains a 2-central element $z$ and, as $z \in Q$, using $Q$ large, yields $C_{G}\left(O_{2}\left(C_{G}(z)\right)\right) \leq O_{2}\left(C_{G}(z)\right)$. This implies that $z$ inverts any normal subgroup of odd order in $C_{G}(y)$ and so such subgroups are abelian. In addition, we prove that $\left|Y_{M}\right| \geq 2^{3}$ and we know $Y_{M} \leq C_{G}(y)$. So signalizer functor methods can be employed 
to obtain $E\left(C_{G}(y)\right) \neq 1$ (see Lemmas 2.3 and 5.2). The arguments used to prove this do not transfer to odd primes as in this case we only find that $O_{p^{\prime}}\left(C_{G}(y)\right)$ is nilpotent and this prevents us demonstrating the balance condition required for use of the signalizer functor theorem.

From among all the components involved in the centralizers of elements in $Y_{M}$ we select one, $K$ say, with first $|K / Z(K)|$ maximal and then $|K|$ maximal. Then from all the elements of $Y_{M}^{\#}$ that contain components we select those $y$ that have $\left|E_{y}\right|$-maximal where $E_{y}$ is the subgroup of $E\left(C_{G}(y)\right)$ generated by components $J$ with $J / Z(J) \cong$ $K / Z(K)$ and $|J|=|K|$. The set of such elements is denoted by $\mathcal{Y}^{*}$ and the members of $\mathcal{Y}^{*}$ are the focus of attention. With these choices, for $y \in \mathcal{Y}^{*}$, Lemma 5.10 shows that, if $C_{S}(y) \in \operatorname{Syl}_{2}\left(C_{G}(y)\right)$ and $\left|C_{S}(y)\right|$ is maximal, then $C_{O_{2}(M)}\left(E_{y}\right)$ is a trivial intersection subgroup of $M$. Roughly speaking, the contradictions which lead to the proof of the Theorem come about by finding that either $M$ normalizes a non-trivial subgroup of $Z(Q)$ which, as $Q$ is large, contradicts $M \not \subset N_{G}(Q)$, or that $M$ is the unique maximal 2-local subgroup containing $S$ which contradicts the fact that $S$ is contained in at least two such subgroups. These two observations are encoded in Lemmas 4.5 and 4.11 .

We give a little more detail, select $y \in \mathcal{Y}^{*}$, fix a component $K \leq E_{y}$ and set $L_{K}=C_{K}(z)$ where $z$ is an involution in $Z(S)$. Then $L_{K}$ has characteristic 2 , and the examination of the various possibilities for $K$ take markedly different routes dependent upon whether or not $L_{K}$ is a 2-group. If $L_{K}$ is not a 2 -group, it is often possible to show that $K=E\left(C_{G}(y)\right)$. Furthermore, Lemma 5.23 asserts that $O^{2}\left(L_{K}\right)$ cannot act irreducibly on $O_{2}(L) / Z\left(O_{2}\left(L_{K}\right)\right.$ ) (the root of this observation lies in Lemma 4.5). This fact eliminates many candidates for $K / Z(K)$. The detailed arguments are presented in Sections 7, 8, , and 10 where, for the more difficult cases, the 2-local structure of $K$ plays a central role in the proof. The data needed for this is provided in Section 3.

By the end of Section 10, we are left with two possibilities. Either $K / Z(K) \cong \mathrm{PSL}_{3}(4)$ or $K \cong \mathrm{Sp}_{4}\left(2^{a}\right)$. Interestingly in this situation we are unable to bound the number of components involved in $E_{y}$. We quickly prove that $Z(K)$ is elementary abelian and that $z \in$ $\Omega_{1}(Z(S)) \leq Y_{M}$ does not project to a root element when $K \cong \operatorname{Sp}_{4}\left(2^{a}\right)$. In Lemma 11.3 we show that the Thompson subgroup of $O_{2}(M)$ is equal to $\left(S \cap E_{y}\right) J\left(C_{S}\left(E_{y}\right)\right)$ and this provides our way into the study of these cases. We eventually show that $M$ either normalizes $E_{y}$, or there is a further subgroup $K_{r+1} \cong K$ which commutes with $E_{y}$ such 
that $M$ normalizes $E_{y} K_{r+1}$. Our objective is to prove that every elementary abelian normal subgroup of $S$ is contained in $Y_{M}$, once this is done the contradiction is provided by Lemma 4.11.

\section{Preliminary group theoretical Results}

In this section we collect some group theoretical facts that we require. In this work we assume that all groups are finite. Recall that for a prime $p$, a group $X$ has characteristic $p$ provided $C_{X}\left(O_{p}(X)\right) \leq O_{p}(X)$ or, equivalently, if $F^{*}(X)=O_{p}(X)$. Our first lemma which is a consequence of coprime action and the Thompson $A \times B$-Lemma [5, Lemma 11.7] is well-known and plays a critical role in our proof of the Theorem.

Lemma 2.1. Suppose that $p$ is a prime, $X$ is a group, $B$ is a $p$-subgroup of $X$ and $C$ is a normal subgroup of $B$. If $N_{X}(C)$ has characteristic $p$, then $N_{X}(B)$ and $C_{X}(B)$ have characteristic $p$.

Proof. Set $A=O_{p^{\prime}}\left(N_{X}(B)\right) E\left(N_{X}(B)\right)$. Then $A$ centralizes $B$ and so $A$ also centralizes $C \leq B$. Therefore $A B \leq N_{X}(C)$ and $A B$ normalizes $P=O_{p}\left(N_{X}(C)\right)$. We have $C_{P}(B)$ normalizes $A$ and, as $[B, A]=1$, $C_{P}(B)$ is normalized by $A$. Hence

$$
\left[C_{P}(B), A\right]=\left[C_{P}(B), A, A\right] \leq\left[O_{p}(A), A\right] \leq\left[Z\left(E\left(N_{X}(B)\right)\right), A\right]=1 .
$$

As $A$ is generated by $p^{\prime}$-elements, the Thompson $A \times B$-Lemma implies that $A$ centralizes $P$ and hence $A=1$ as $N_{X}(C)$ has characteristic $p$. Therefore $F^{*}\left(N_{X}(B)\right)=O_{p}\left(N_{X}(B)\right)$ and so $N_{X}(B)$ has characteristic $p$. Since $F^{*}\left(C_{X}(B)\right) \leq F^{*}\left(N_{X}(B)\right)$, we also have $C_{X}(B)$ has characteristic $p$.

As an example of how we might use Lemma 2.1 consider the case $X$ has characteristic $p$. Then we may take $C=1$, and obtain $N_{X}(B)$ has characteristic $p$.

Lemma 2.2. Let $X$ be a group of characteristic $p$ and $Y$ be subnormal in $X$. Then $Y$ is a group of characteristic $p$.

Proof. If $Y$ is subnormal in $X$, then $F^{*}(Y) \leq F^{*}(X)$. Hence $F^{*}(Y)$ is a $p$-group.

The next lemma will be used to show that certain involutions have components in their centralizers.

Lemma 2.3. Suppose that $X$ is a group and $Y$ is an elementary abelian 2-subgroup of $X$ of order at least 8. Assume that $E\left(C_{X}(x)\right)=1$ for all $x \in Y^{\#}$ and that there exists $z \in Y^{\#}$ such that $F^{*}\left(C_{X}(z)\right)=$ $\mathrm{O}_{2}\left(C_{X}(z)\right)$. Then $\left\langle O\left(C_{X}(y)\right) \mid y \in Y^{\#}\right\rangle$ has odd order and is normalized by $N_{X}(Y)$. 
Proof. Suppose that $a, b \in Y^{\#}$ are such that $F^{*}\left(C_{X}(a)\right)=O_{2}\left(C_{X}(a)\right)$ and $O\left(C_{X}(b)\right) \neq 1$. Then $C_{C_{X}(a)}(b)=C_{C_{X}(b)}(a)$ has characteristic 2 by Lemma 2.1. In particular, $C_{O\left(C_{X}(b)\right)}(a)=1$ and so $a$ inverts $O\left(C_{X}(b)\right)$. This means that $O\left(C_{X}(b)\right)$ is abelian. Since there exists $z \in Y^{\#}$ such that $F^{*}\left(C_{X}(z)\right)=O_{2}\left(C_{X}(z)\right)$, we have $O\left(C_{X}(b)\right)$ is abelian and is inverted by $z$ for all $b \in Y^{\#}$.

Suppose that $a, b \in Y^{\#}$ are arbitrary. We claim that

$$
O\left(C_{X}(b)\right) \cap C_{X}(a) \leq O\left(C_{X}(a)\right) .
$$

If $F^{*}\left(C_{X}(b)\right)=O_{2}\left(C_{X}(b)\right)$, then $O\left(C_{X}(b)\right)=1$ and there is nothing to prove. Suppose that $F^{*}\left(C_{X}(a)\right)=O_{2}\left(C_{X}(a)\right)$, then we have already argued that $O\left(C_{X}(b)\right) \cap C_{X}(a)=1$ and so the claimed containment also holds in this case. Suppose that $O\left(C_{X}(b)\right) \neq 1 \neq O\left(C_{X}(a)\right)$. Set $U=O\left(C_{X}(b)\right) \cap C_{X}(a)$. Then $\langle b\rangle \times U$ normalizes $O_{2}\left(C_{X}(a)\right)$ and $\left[C_{O_{2}\left(C_{X}(a)\right)}(b), U\right] \leq O_{2}\left(C_{X}(a)\right) \cap O\left(C_{X}(b)\right)=1$. Thus again the Thompson $A \times B$-Lemma implies that $\left[U, O_{2}\left(C_{X}(a)\right)\right]=1$. Now consider $U O\left(C_{X}(a)\right)$. This group is normalized by $z$ and, as $z$ inverts $U$ and inverts $O\left(C_{X}(a)\right)$, we have $z$ inverts $U O\left(C_{X}(a)\right)$. But then $U O\left(C_{X}(a)\right)$ is abelian. Consequently $U$ centralizes $F^{*}\left(C_{X}(a)\right)=O\left(C_{X}(a)\right) O_{2}\left(C_{X}(a)\right)$ and so $U \leq O\left(C_{X}(a)\right)$ as claimed.

As $|Y| \geq 8$ by hypothesis, the Soluble Signalizer Functor Theorem [5, Theorem 21.3] implies that the completeness subgroup

$$
\Sigma=\left\langle O\left(C_{X}(b)\right) \mid b \in Y_{M}^{\#}\right\rangle
$$

has odd order. Finally we note that $N_{X}(Y)$ normalizes $\Sigma$ as it permutes the generating subgroups by conjugation. This completes the proof of the lemma.

Recall from [5, Definition 4.5] that a 2-component of a group $X$ is a subnormal perfect subgroup $F$ of $X$ such that $F / O(F)$ is quasisimple. The subgroup $L_{2^{\prime}}(X)$ is defined to be the subgroup of $X$ generated by all the 2-components of $X$ and is called the 2-layer of $X$. The subgroup $X^{\infty}$ of $X$ is the last member in the derived series of $X$.

Lemma 2.4. We have

$$
C_{L_{2^{\prime}}(X)}\left(O\left(L_{2^{\prime}}(X)\right)\right)=E(X) Z\left(O\left(L_{2^{\prime}}(X)\right)\right) O_{2}\left(L_{2^{\prime}}(X)\right) .
$$

Proof. Plainly $C_{L_{2^{\prime}}(X)}\left(O\left(L_{2^{\prime}}(X)\right)\right) \geq E(X) Z\left(O\left(L_{2^{\prime}}(X)\right)\right) O_{2}\left(L_{2^{\prime}}(X)\right)$. We may as well suppose that $X=L_{2^{\prime}}(X)$. Set $\bar{X}=X / O(X)$ and $C=C_{X}(O(X))$. Then $\bar{X}=E(\bar{X})$ by [5, Proposition 4.7 (iii)]. Therefore $\bar{C}$ is a product of components of $\bar{X}$ together with $\overline{O_{2}(X)}$. Assume that $K \leq C$ is such that $\bar{K}$ is a component in $\bar{C}$. Then $K O(X)$ is 
normal in $X$ and $K^{\infty}$ is a 2-component of $X$. If $K^{\infty}$ is not a component of $X$, then $O\left(K^{\infty}\right) \not \leq Z\left(K^{\infty}\right)$. As $K \leq C$, this is impossible. Hence $K \leq E(X)$. Thus $\bar{C}=\overline{E(X) O_{2}(X)}$ and consequently $C \leq E(X) O_{2}(X) O(X)$. Using the Dedekind Modular Law we obtain

$$
\begin{aligned}
C & =C \cap E(X) O_{2}(X) O(X)=E(X) O_{2}(X)(C \cap O(X)) \\
& =E(X) O_{2}(X) Z(O(X)),
\end{aligned}
$$

as claimed.

Lemma 2.5. Suppose that $K$ is a component of the group $X$ and $T \in$ $\operatorname{Syl}_{2}(X)$. If $Y$ is an abelian normal subgroup of $T$ and $Y$ does not normalize $K$, then $K / Z(K)$ has abelian Sylow 2-subgroups.

Proof. See [14, Lemma 2.28].

Lemma 2.6. Assume that $R \leq G$ be a 2-group which normalizes the subgroup $P \leq N_{G}(R)$. Set $V=\left[O_{2}(P), O^{2}(P)\right]$ and assume that the non-central $O^{2}(P)$-chief factors in $V / \Phi(V)$ are pairwise non-isomorphic. Then $[V, R] \leq \Phi(V)$.

Proof. Set $\bar{V}=V / \Phi(V)$. As $R$ normalizes $P, R$ operates on $\bar{V}$ and, as $R$ is normalized by $P$, coprime action yields

$$
\left[R, O^{2}(P)\right]=\left[R, O^{2}(P), O^{2}(P)\right] \leq\left[O_{2}(P), O^{2}(P)\right]=V \leq C_{O_{2}(P)}(\bar{V}) .
$$

Assume $R>C_{R}(\bar{V})$ and select $x \in R \backslash C_{R}(\bar{V})$ such that $x^{2} \in C_{R}(\bar{V})$. Then $\bar{V}>C_{\bar{V}}(x)$ and $[\bar{V}, x] \neq 1$ are $O^{2}(P)$-invariant as $\left[x, O^{2}(P)\right]$ centralizes $\bar{V}$. Additionally, $\bar{V} / C_{\bar{V}}(x) \cong[\bar{V}, x]$ as $O^{2}(P)$-modules. In addition, as $x^{2} \in C_{R}(V)$, we have $C_{\bar{V}}(x) \geq[\bar{V}, x]$. Thus, the condition on the non-central $O^{2}(P)$-chief factors in $\bar{V}$ implies that $\bar{V} / C_{\bar{V}}(x)$ is centralized by $O^{2}(P)$. But then $V=\left[V, O^{2}(P)\right]<V$, a contradiction. Hence $R=C_{R}(\bar{V})$ as claimed.

We recall that the Thompson subgroup $J(X)$ of a group $X$, is the subgroup of $X$ generated by the elementary abelian subgroups of $X$ of maximal rank. One of the main tools in the proof of the theorem of this paper requires that we locate $J\left(O_{2}(M)\right)$ in certain subgroups of centralizers of elements in $Y_{M}$. The next two results are important when such subgroups have more than one component.

Proposition 2.7. Suppose that $X$ is a group, $O(X)=1, K$ is a component of $X$ and $S$ is a Sylow 2-subgroup of $X$. Assume that $K$ satisfies the following two properties.

(i) For $\bar{x} \in K / Z(K)$ an involution, there is a preimage $x$ such that

(a) $x$ is an involution; and 
(b) any involution in $\operatorname{Aut}(K)$, which centralizes $\bar{x}$ also centralizes $x$.

(ii) If $K / Z(K)$ has dihedral or semidihedral Sylow 2-subgroups, then $\operatorname{Aut}(K / Z(K))$ does not contain a fours-group disjoint from $\operatorname{Inn}(K / Z(K))$.

Then $J(S)$ normalizes $K$. In particular, if $K \in \mathcal{K}$ is simple, then $J(S)$ normalizes $K$.

Proof. This is [5, Proposition 8.5] and the remark thereafter.

Lemma 2.8. Suppose $G=S E$ where $S \in \operatorname{Syl}_{2}(G)$ and $E$ is a direct product of simple components $K \in \mathcal{K}$ of $G$. Assume that each component $K$ of $E$ satisfies

$$
\text { if } T \in \operatorname{Syl}_{2}(\operatorname{Aut}(K)) \text {, then } J(T)=J(T \cap \operatorname{Inn}(K)) \text {. }
$$

Then

$$
J(S)=J\left(C_{S}(E)\right) \times J(S \cap E)
$$

and

$$
J(S \cap E)=\prod_{K \text { a component of } G} J(S \cap K) .
$$

Proof. Assume that $A$ is an elementary abelian 2-subgroup of $S$ of maximal rank. By Proposition [2.7 every component $K$ of $G$ is normalized by $A$. Furthermore $A C_{G}(K) / C_{G}(K)$ is an elementary abelian 2 -subgroup of $\operatorname{Aut}(K)$. Assume that $A C_{G}(K) / C_{G}(K)$ is not a maximal rank elementary abelian 2-subgroup of $N_{G}(K) / C_{G}(K)$. Then, by (1), there exists an elementary abelian $p$-subgroup $B \leq K$ such that

$$
|B|=\left|B C_{G}(K) / C_{G}(K)\right|>\left|A C_{G}(K) / C_{G}(K)\right|=\left|A: A \cap C_{G}(K)\right| .
$$

Set $B_{0}=B\left(A \cap C_{G}(K)\right)$. Then $B_{0}$ is elementary abelian and $\left|B_{0}\right|=$ $|B|\left|A \cap C_{G}(K)\right|>|A|$, contrary to the choice of $A$. Hence $A C_{G}(K) / C_{G}(K)$ is a maximal rank elementary abelian 2-subgroup of $N_{G}(K) / C_{G}(K)$ and therefore $A \leq K C_{G}(K)$ and $A=(A \cap K)\left(A \cap C_{G}(K)\right)$ with $A \cap K$ a maximal rank elementary abelian 2-subgroup of $K$. Assume that $E=K_{1} \cdots K_{\ell}$. Then, for $1 \leq j \leq \ell$, we have shown that

$$
A=C_{A}\left(K_{j}\right)\left(A \cap K_{j}\right)
$$

where $A \cap K_{j}$ is a maximal rank elementary abelian 2-subgroup of $K_{j}$ and $C_{A}\left(K_{j}\right)$ is a maximal rank elementary abelian 2-subgroup of 
$C_{S}\left(K_{j}\right)$. Notice that by the Modular Law

$$
\begin{aligned}
A & =C_{A}\left(K_{1}\right)\left(A \cap K_{1}\right) \cap C_{A}\left(K_{2}\right)\left(A \cap K_{2}\right) \\
& =\left(C_{A}\left(K_{1}\right)\left(A \cap K_{1}\right) \cap C_{A}\left(K_{2}\right)\right)\left(A \cap K_{2}\right) \\
& =\left(C_{A}\left(K_{1}\right) \cap C_{A}\left(K_{2}\right)\right)\left(A \cap K_{1}\right)\left(A \cap K_{2}\right) \\
& =C_{A}\left(K_{1} K_{2}\right)\left(A \cap K_{1}\right)\left(A \cap K_{2}\right)
\end{aligned}
$$

and continuing in this way yields $A=C_{A}(E)\left(A \cap K_{1}\right) \ldots\left(A \cap K_{\ell}\right)$. This proves the claim.

Remark 2.9. If $K$ is a simple group, then the statement in Proposition 2.7 can be proved for all primes $p$ provided $O_{p^{\prime}}(X)=1$, where we do not need $K \in \mathcal{K}$ for $p>2$. The statement of Lemma 2.8 also holds for all primes.

\section{Properties of $\mathcal{K}$-groups}

We require detailed information about the 2-local structure of certain of the groups of Lie type defined in characteristic 2 . What we require can mostly be found in [13, but we present the statements here for the convenience of the reader. We start with groups defined over a field of characteristic 2. In the next lemma we use the notation $V_{n}$ to denote a natural module for a classical group defined in dimension $n$ but considered as a GF(2)-module. Thus, if $X$ is a classical group defined over $\operatorname{GF}\left(2^{e}\right)$, then $\left|V_{n}\right|=2^{\text {ne }}$.

Lemma 3.1. Let $X$ be a simple group of Lie type defined in characteristic 2 and $R$ be a long root subgroup of $X$. Set $Q=O_{2}\left(N_{X}(R)\right)$

\begin{tabular}{|c|c|c|c|}
\hline$X$ & $L / Z(L)$ & $m_{2}(Q / R)$ & $Q / R$ \\
\hline $\operatorname{PSL}_{m}\left(2^{e}\right), m \geq 5$ & $\mathrm{PSL}_{m-2}\left(2^{e}\right)$ & $2(m-2) e$ & $V_{m-2} \oplus V_{m-2}^{*}$ \\
\hline $\mathrm{PSU}_{m}\left(2^{e}\right), m \geq 5$ & $\mathrm{PSU}_{m-2}\left(2^{e}\right)$ & $(m-2) 2 e$ & $V_{m-2}$ \\
\hline $\mathrm{P} \Omega_{2 m}^{ \pm}\left(2^{e}\right), m \geq 4$ & $\mathrm{PSL}_{2}\left(2^{e}\right) \times \mathrm{P} \Omega_{2(m-2)}^{ \pm}\left(2^{e}\right)$ & $4(m-2) e$ & $V_{2} \otimes V_{2 m-4}$ \\
\hline $\mathrm{P} \Omega_{6}^{ \pm}\left(2^{e}\right)$ & $\mathrm{PSL}_{2}\left(2^{e}\right)$ & $4 e$ & $V_{2} \oplus V_{2}$ \\
\hline $\mathrm{E}_{6}\left(2^{e}\right)$ & $\operatorname{PSL}_{6}\left(2^{e}\right)$ & $20 e$ & \\
\hline${ }^{2} \mathrm{E}_{6}\left(2^{e}\right)$ & $\mathrm{PSU}_{6}\left(2^{e}\right)$ & $20 e$ & \\
\hline $\mathrm{E}_{7}\left(2^{e}\right)$ & $\mathrm{P} \Omega_{12}^{+}\left(2^{e}\right)$ & $32 e$ & \\
\hline $\mathrm{E}_{8}\left(2^{e}\right)$ & $\mathrm{E}_{7}\left(2^{e}\right)$ & $56 e$ & \\
\hline $\mathrm{G}_{2}\left(2^{e}\right), e \geq 3$ & $\mathrm{PSL}_{2}\left(2^{e}\right)$ & $4 e$ & \\
\hline${ }^{3} \mathrm{D}_{4}\left(2^{e}\right)$ & $\mathrm{PSL}_{2}\left(2^{3 e}\right)$ & $8 e$ & \\
\hline
\end{tabular}
and $L=O^{2}\left(N_{X}(R) / Q\right)$. Then for specified $X$, the following table displays the Levi section $L / Z(L)$, the 2 -rank of $Q / R$ and, for the classical groups $X$, describes the action of $L$ on $Q / R$.

Furthermore, other than for $X \cong \mathrm{PSL}_{m}\left(2^{e}\right)$ and $\mathrm{P} \Omega_{6}^{ \pm}\left(2^{e}\right), Q / R$ is an irreducible L-module and, for the exceptional groups, it is defined over $\mathrm{GF}\left(2^{e}\right)$. If $X \cong \mathrm{P} \Omega_{6}^{-}\left(2^{e}\right)$, then $C_{X}(R)$ acts irreducibly on $Q / R$. 
Proof. This is [13, Lemmas D.1 and D.10].

Lemma 3.2. Let $X \cong \mathrm{PSL}_{4}\left(2^{a}\right)$ with $a>1, R$ be a root subgroup of $X, L=C_{X}(R)$ and $Q=O_{2}(L)$. Then the non-central chief factors of $Q / R$ are not isomorphic as $L$-modules.

Proof. This is checked by direct calculation. Let $\lambda$ be a primitive element in $\operatorname{GF}\left(2^{a}\right)$ and put $\delta=\operatorname{diag}\left(\lambda, \lambda^{-2}, 1, \lambda\right)$. Then $\delta$ is non-central in $X$ and centralizes $Z(S)$, where $S$ is taken to be the subgroup of lower unitriangular matrices. Let

$$
E_{1}=\left\{\left(\begin{array}{llll}
1 & 0 & 0 & 0 \\
\alpha & 1 & 0 & 0 \\
\beta & 0 & 1 & 0 \\
\gamma & 0 & 0 & 1
\end{array}\right) \mid \alpha, \beta, \gamma \in \mathrm{GF}\left(2^{a}\right)\right\}
$$

and

$$
E_{2}=\left\{\left(\begin{array}{llll}
1 & 0 & 0 & 0 \\
0 & 1 & 0 & 0 \\
0 & 0 & 1 & 0 \\
\gamma & \beta & \alpha & 1
\end{array}\right) \mid \alpha, \beta, \gamma \in \mathrm{GF}\left(2^{a}\right)\right\} .
$$

Then $Q=E_{1} E_{2}$ and we calculate that conjugation of $E_{1}$ by $\delta$ scales $\beta$ by $\lambda$ and conjugation of $E_{2}$ by $\delta$ leaves $\beta$ unchanged. It follows that the $\langle\delta\rangle$-invariant subgroups of $Z_{2}(S)$ are in $Z_{2}(S) \cap E_{1}$ or $Z_{2}(S) \cap E_{2}$. From this we deduce that $E_{1}$ and $E_{2}$ are the only normal subgroups of $L$ contained in $Q$ which have order $2^{3 a}$. This proves the result.

Lemma 3.3. Suppose that $K \cong \mathrm{SL}_{2}\left(2^{e+1}\right)$ or ${ }^{2} \mathrm{~B}_{2}\left(2^{2 e+1}\right)$ with $e \geq 1$. Let $T \in \mathrm{Syl}_{2}(\operatorname{Aut}(K))$. Then either $K \cong \mathrm{SL}_{2}\left(2^{2}\right)$ or $J(T)=J(T \cap$ $\operatorname{Inn}(K))=\Omega_{1}(T \cap \operatorname{Inn}(K))$.

Proof. We identify $K$ with $\operatorname{Inn}(K)$. If $K$ is a Suzuki group then $T \leq K$ by [6. Theorem 2.5.12] and $\Omega_{1}(T \cap K)=Z(T \cap K)$ and we are done. So suppose that $K \cong \mathrm{SL}_{2}\left(2^{e+1}\right)$. Let $x \in T \backslash K$ be an involution. Then $x$ acts as a field automorphism on $K$ and $e+1$ is even. Thus $C_{K}(x) \cong \mathrm{SL}_{2}\left(2^{(e+1) / 2}\right)$ by [․ Theorem 4.9.1].

Assume that $A \leq T$ has maximal rank. Then $|A| \geq 2^{e+1}$ and $T \cap K$ is elementary abelian of order $2^{e+1}$. Assume $A \not \leq T$. Then

$$
1+(e+1) / 2 \geq e+1
$$

as $K$ has 2 -rank $e+1$. Hence either $K \cong \mathrm{SL}_{2}(4)$ or $J(T) \leq K$. In the latter case, we have $J(T)=J(T \cap K)=\Omega_{1}(T \cap K)=T \cap K$.

We need the following well-known result about representations of $\mathrm{SL}_{2}\left(2^{e}\right)$.

Lemma 3.4. Let $V$ be a non-split extension of a trivial module by the natural module for $X=\mathrm{SL}_{2}\left(2^{e}\right)$. Let $S$ be a Sylow 2-subgroup of $X$ and $A$ be a fours-group in $S$. Then $[V, A]=[V, S]$. 
Proof. By a result of Gaschütz [8, Satz I.17.4], we may assume that $C_{V}(X) \leq[V, S]$. Hence, if $[V, A] \neq[V, S]$, as $\left[V / C_{V}(X), S\right]=\left[V / C_{V}(X), A\right]$, there is a hyperplane in $C_{V}(X)$ which contains $[V, A] \cap C_{V}(X)$. Thus we may assume that $\left|C_{V}(X)\right|=2$. Choose $\nu \in X$, of order $q+1$ and $\nu^{a}=\nu^{-1}$ for some $a \in A$. We have that $|[V, \nu]|=q^{2}$ has index 2 in $V$ and $V=[V, \nu]+C_{V}(X)$. Therefore $[V, a] \leq[V, \nu]$. Let $A=\langle a, b\rangle$. We have that $[V, \nu]+[V, b]$ is invariant under $\langle A, \nu\rangle=X$. Hence $[V, \nu]+[V, b]=V$ and so $[V, A]>[V, a]$, which implies $C_{V}(X) \leq[V, A]$ and then $[V, A]=[V, S]$.

Lemma 3.5. Suppose that $X \cong \operatorname{Sp}_{2 n}(q)$ with $q=2^{e}$ and $n \geq 3$, and let $R_{1}$ be a long root subgroup and $R_{2}$ be a short root subgroup of $X$. For $i=1,2$, set $Q_{i}=O_{2}\left(N_{X}\left(R_{i}\right)\right)$ and

$$
L_{i}=O^{2^{\prime}}\left(N_{X}\left(R_{i}\right) / Q_{i}\right) \text {. }
$$

Then

(i) $L_{1} \cong \operatorname{Sp}_{2 n-2}(q), Q_{1}$ is elementary abelian and $Q_{1} / R_{1}$ is a natural $\mathrm{Sp}_{2 n-2}(q)$-module; and

(ii) $L_{2} \cong \operatorname{Sp}_{2 n-4}(q) \times \mathrm{SL}_{2}(q), \Phi\left(Q_{2}\right)=Q_{2}^{\prime}=R_{2}, Z\left(Q_{2}\right) / R_{2}$ is a natural $\mathrm{SL}_{2}(q)$-module and $Q_{2} / Z\left(Q_{2}\right)$ is the tensor product of natural modules of the direct factors of $L_{2}$. In addition, if $q>2$, then $Z\left(Q_{2}\right)$ does not split over $R_{2}$ as an $L_{2}$-module.

Proof. See [13, Lemma D.5].

Lemma 3.6. Suppose that $X \cong \operatorname{Sp}_{2 n}(q), q=2^{e}$, with $n \geq 3$. Let $V$ be the natural symplectic module, $P$ be the stabilizer of a maximal isotropic subspace of $V$ and $S \in \mathrm{Syl}_{2}(P)$. Then $J(S)=\mathrm{O}_{2}(P)$ is elementary abelian.

Proof. See [13, Lemma D.6].

Lemma 3.7. Suppose that $X \cong \operatorname{PSp}_{4}(q), q=2^{e}>2$, let $T$ be a Sylow 2-subgroup of $\operatorname{Aut}(X)$ and set $S=T \cap X$. Then $X$ has exactly two parabolic subgroups $P_{1}, P_{2}$ which contain $S$. For $i=1,2, E_{i}=O_{2}\left(P_{i}\right)$ is elementary abelian of order $q^{3}$ and $P_{i} / E_{i} \cong \mathrm{GL}_{2}(q)$. We have that $E_{i}$ is an indecomposable module for $P_{i}$ and $Z\left(O^{2^{\prime}}\left(P_{i}\right)\right)=R_{i}$ is a root group. Furthermore $Z(S)=R_{1} R_{2}=S^{\prime}, J(T)=J(S)=S=E_{1} E_{2}$ and any involution in $S$ is contained in $E_{1} \cup E_{2}$.

Proof. See [13, Lemmas D.3 and D.4].

Lemma 3.8. Suppose that $X \cong \operatorname{PSp}_{4}(q), q=2^{e}>2$, and $S \in \operatorname{Syl}_{2}(X)$. If $D$ is a non-abelian normal subgroup of $S$, then either $Z(S) \leq D$ or $C_{S}(D)=Z(S)$ and $\left|D E_{1} / E_{1}\right|=\left|D E_{2} / E_{2}\right|=2$. 
Proof. We use the notation from Lemma 3.7. Assume that $Z(S) \not \subset D$. Then $\left|D E_{1} / E_{1}\right|=\left|D E_{2} / E_{2}\right|=2$ for otherwise $Z(S)=\left[E_{1}, D\right]$ by Lemma 3.4. Since $D$ is non-abelian, $|D Z(S) / Z(S)| \geq 4$. Hence there exists $t_{i} \in\left(E_{i} \cap D\right) \backslash Z(S)$ for $i=1,2$. As $C_{E_{3-i}}\left(t_{i}\right)=Z(S)$ by the last line of Lemma 3.7, we have $C_{S}\left(t_{i}\right)=E_{i}$. Therefore $C_{S}(D) \leq E_{1} \cap E_{2}=$ $Z(S)$.

Lemma 3.9. Suppose that $X$ is quasisimple and $X / Z(X) \cong \mathrm{PSL}_{3}(4)$ and $S \in \operatorname{Syl}_{2}(X)$. If $Z(X)$ has an element of order 4 , then $Z(S) \leq$ $Z(X)$.

Proof. See [7, Chapter 10, Lemma 2.3 (a)].

Lemma 3.10. Suppose that $X$ is a group with $F^{*}(X) \cong \operatorname{PSL}_{3}\left(2^{e}\right)$, $e \geq 1$. Let $T \in \operatorname{Syl}_{2}(X)$ and $S=T \cap F^{*}(X)$. Then

(i) $F^{*}(X)$ possesses exactly two parabolic subgroups $P_{1}, P_{2}$ which contain $S$. For $i=1,2, E_{i}=O_{2}\left(P_{i}\right)$ is elementary abelian of order $2^{2 e}, O^{2^{\prime}}\left(P_{i} / E_{i}\right) \cong \mathrm{SL}_{2}\left(2^{e}\right)$ and $E_{i}$ is a natural module for $O^{2^{\prime}}\left(P_{i}\right)$. Furthermore $S=E_{1} E_{2}$ and any involution in $S$ is contained in $E_{1} \cup E_{2}$.

(ii) every elementary abelian normal subgroup of $T$ is contained in $S$;

(iii) $J(T)=J(S)=E_{1} E_{2}$.

Proof. See [13, Lemmas D.2 and D.4].

Lemma 3.11. Suppose that $X$ is a group of Lie type in characteristic 2. If $\sigma$ is an automorphism of $X$ of order 2 which centralizes a Sylow 2 -subgroup of $X$, then either $\sigma$ is inner or $X \cong \operatorname{PSp}_{4}(2)^{\prime}$.

Proof. This follows from [3, Chapter 19] when $X \neq^{2} \mathrm{~F}_{4}(q)$. For $X \cong$ ${ }^{2} \mathrm{~F}_{4}(q)$ we can use [6, Theorem 9.1] for $q>2$ and for $q=2$ the result follows from [6, Theorems 2.5.12, 2.5.15 and 3.3.2].

Lemma 3.12. Suppose that $X$ is a group with $F^{*}(X) \cong \operatorname{PSL}_{3}\left(2^{e}\right)$, $e \geq 1$. Let $T \in \operatorname{Syl}_{2}(X)$ and $S=T \cap F^{*}(X)$. Then $C_{T}(S)=Z(S)$.

Proof. Set $Y=C_{T}(S)$. Then $Y$ is normalized by $B=N_{F^{*}(X)}(S)$. Let $C$ be a Cartan subgroup of $B$, then $Y=C_{Y}(C)[Y, C]$ and $[Y, C]=Z(S)$. In particular, if $C_{Y}(C) \neq 1$, then $C_{Y}(C)$ contains an involution. This contradicts Lemma 3.11.

Lemma 3.13. Suppose that $X$ is quasisimple with $X / Z(X) \cong \mathrm{PSL}_{3}(4)$ and $Z(X)$ elementary abelian. Let $T \in \operatorname{Syl}_{2}(\operatorname{Aut}(X)), S=T \cap X \in$ $\operatorname{Syl}_{2}(X)$ and $\bar{X}=X / Z(X)$. 
(i) $\bar{S}$ has exactly two elementary abelian subgroups $\overline{E_{1}}$ and $\overline{E_{2}}$ of order 16. Every involution of $\bar{S}$ is in $\overline{E_{1}} \cup \overline{E_{2}}, \bar{S}=\overline{E_{1} E_{2}}=$ $J(\bar{T})=\overline{J(T)}$ and $C_{\bar{S}}(x)=\overline{E_{i}}$ for all $x \in \overline{E_{i}} \backslash Z(\bar{S})$. For $i=1,2$, let $E_{i}$ be the preimage of $\overline{E_{i}}$. Then $E_{i}$ is elementary abelian.

(ii) $\left[S, E_{1}\right]=\left[S, E_{2}\right]=S^{\prime}=Z(S)=E_{1} \cap E_{2} \geq Z(X)$.

(iii) If $D$ is a non-abelian normal subgroup of $S$, then $[\bar{S}, \bar{D}]=$ $Z(\bar{S})=\overline{Z(S)}=C_{\bar{S}}(\bar{D})$.

(iv) Every normal elementary abelian subgroup of $T$ is contained in $S$.

Proof. For part (i) and (iv) see Lemma 3.10 and [7, Chapter 10, Lemma $2.1(\mathrm{~h})$ and 2.2].

We now prove (ii). Since $E_{1}$ is elementary abelian, we may regard it as a $\mathrm{GF}(2) H$-module for $H=N_{X}\left(E_{1}\right)$. As $E_{1}$ centralizes $\overline{E_{1}}$, Lemma 3.10 implies that $N_{X}\left(E_{1}\right)$ induces the natural $\mathrm{SL}_{2}(4)$-module on $\overline{E_{1}}$. We claim $\left[E_{1}, S\right]=Z(S) \geq Z(X)$. Certainly we have

$$
\left[E_{1}, S\right]=\left[E_{1}, E_{1} E_{2}\right]=\left[E_{1}, E_{2}\right] \leq E_{1} \cap E_{2} \leq Z(S)
$$

and $\left[E_{1}, S\right] Z(X)=E_{1} \cap E_{2}=Z(S)$. To prove that $Z(X) \leq\left[E_{1}, S\right]$, we may suppose that $|Z(X)|=2$. Suppose that $\left[E_{1}, S\right]<E_{1} \cap E_{2}$. Then $\left[E_{1}, S\right] \cap Z(X)=1$. For $x \in N_{X}\left(E_{1}\right) \backslash N_{X}(S)$, we have $N_{X}\left(E_{1}\right)=\left\langle S, S^{x}\right\rangle$ and so $\left[E_{1}, S\right]\left[E_{1}, S^{x}\right]$ as order $2^{4}$ and is normalized by $N_{X}\left(E_{1}\right)$. Since $E_{2}$ is elementary abelian, we obtain $S /\left[E_{1}, S\right]\left[E_{1}, S^{x}\right]$ is elementary abelian. Hence $N_{X}\left(E_{1}\right) /\left[E_{1}, S\right]\left[E_{1}, S^{x}\right]$ splits as $2 \times \mathrm{SL}_{2}(4)$. It follows that $S$ splits over $Z(X)$ and we have a contradiction via Gaschütz's Theorem [8, (I.17.4)]. Hence (ii) holds.

For (iii), suppose that $D$ is a non-abelian normal subgroup of $S$. Then $D \not E_{1}$ and so $\left[\overline{E_{1}}, \bar{D}\right]=Z(\bar{S})=\overline{Z(S)}$ as $\overline{E_{1}}$ is a natural $N_{X}\left(E_{1}\right) / E_{1^{-}}$ module. We now determine $C_{\bar{S}}(\bar{D})$. We have that $\bar{D}$ has order at least 16 and $\bar{D}$ contains $Z(\bar{S})$. If $\bar{D} \cap \overline{E_{1}}>Z(\bar{S})$, then $C_{\bar{S}}(\bar{D}) \leq C_{\bar{E}_{1}}(\bar{D})=$ $Z(\bar{S})$, the assertion. So assume $\bar{D} \cap \overline{E_{1}}=Z(\bar{S})$. Then $S=D E_{1}$ and $|\bar{D}|=16$. Thus we can apply Lemma 3.4 to see that $D \geq\left[E_{1}, D\right]=$ $\left[E_{1}, S\right]=Z(S)$. In particular $\overline{Z(S)}=Z(\bar{S})=Z(\bar{D})=C_{\bar{S}}(\bar{D})$, as claimed.

Lemma 3.14. Let $X$ be quasisimple with $X / Z(X) \cong \mathrm{PSL}_{3}(4)$ and $Z(X)$ elementary abelian. Then $X$ satisfies assumption (i) of Proposition 2.7.

Proof. By [6, Corollary 5.1.4] we can lift $\operatorname{Aut}(X)$ to a group of automorphisms of the universal covering group of $X / Z(X)$ and then restrict it to a group $X_{1}$ such that $\left|Z\left(X_{1}\right)\right|$ is elementary abelian of order 4 and 
$X_{1} / Z\left(X_{1}\right) \cong X / Z(X)$. Hence it is enough to prove the assertion when $|Z(X)|=4$.

We follow the notation in Lemma 3.13. Set $P=N_{X}\left(E_{1}\right)$. Since $E_{1}$ is elementary abelian by Lemma 3.13 (ii) and $X / Z(X)$ has just one conjugacy class of involutions, there are no elements of $X$ of order 4 with square in $Z(X)$. This is the condition (i)(a) of Proposition 2.7.

Assume that $\bar{x}$ is an involution in $X / Z(X)$ and let $Y$ be the preimage of $\langle\bar{x}\rangle$. Then $Y$ is elementary abelian of order 8 . We will show that there is some $x \in Y \backslash Z(X)$ which is centralized by any automorphism of $X$ centralizes $\bar{x}$. From [6, Table 6.3.1] we know $\operatorname{Out}(X / Z(X)) \cong$ $\operatorname{Sym}(3) \times 2$ and acts on $Z(X)$ with an element of order three nontrivial. Since $\operatorname{Inn}(X)$ acts transitively on the involutions in $X / Z(X)$, $N_{\text {Aut }(X)}(Y) \operatorname{Inn}(X)=\operatorname{Aut}(X)$. As $C_{\operatorname{Inn}(X)}(Y)=\bar{T}$, and $|Y|=2^{3}$, the subgroup structure of $\mathrm{SL}_{3}(2)$ yields $N_{\text {Aut }(X)}(Y) / C_{\text {Aut }(X)}(Y) \cong \operatorname{Sym}(3)$. Let $\rho \in N_{\text {Aut }(X)}(Y)$ have order three. Then $Y=[Y, \rho] \times\langle x\rangle$ and $\langle x\rangle$ is centralized by $N_{\operatorname{Aut}(X)}(Y)$. Thus $x$ is a preimage of $\bar{x}$, which is centralized by any automorphism which normalizes $Y$. This element satisfies the assumption (i)(b) of Proposition 2.7.

Lemma 3.15. Suppose that $X \cong \mathrm{F}_{4}(q)$ with $q=2^{e}$ and let $R_{1}$ be a long root subgroup and $R_{2}$ be a short root subgroup of $X$. For $i=1,2$, set $Q_{i}=O_{2}\left(N_{X}\left(R_{i}\right)\right)$ and $L_{i}=O^{2^{\prime}}\left(N_{X}\left(R_{i}\right) / Q_{i}\right)$. Then, for $i=1,2$, we have $L_{i} \cong \operatorname{Sp}_{6}(q)$ and $\Phi\left(Q_{i}\right)=R_{i}$. Furthermore, as $L_{i}$-modules, $Z\left(Q_{i}\right) / R_{i}$ is a natural module of dimension $6, Q_{i} / Z\left(Q_{i}\right)$ is a spin module of dimension 8 and the modules $Z\left(Q_{i}\right)$ and $Q_{i} / R_{i}$ are indecomposable.

Proof. See [13, Lemma D.7].

Lemma 3.16. Suppose that $X \cong \mathrm{F}_{4}(q)$ with $q=2^{e}, S \in \operatorname{Syl}_{2}(X)$ and $\Omega_{1}(Z(S))=R_{1} R_{2}$ with $R_{1}$ a long root subgroup of $X$ and $R_{2}$ a short root subgroup of $X$. We use the notation introduced in Lemma 3.15 and additionally set $I_{12}=C_{X}\left(R_{1} R_{2}\right), Q_{12}=O_{2}\left(I_{12}\right)$ and $L_{12}=I_{12} / Q_{12}$. For $i=1,2$, define

$$
V_{i}=\left[Z\left(Q_{i}\right), Q_{12}\right] R_{1} R_{2},
$$

put $V_{12}=V_{1} V_{2}$ and $W_{12}=Z\left(Q_{1}\right) Z\left(Q_{2}\right)$.

Then the following hold:

(i) $L_{12} \cong \operatorname{Sp}_{4}(q)$ and $Q_{12}=Q_{1} Q_{2}$.

(ii) $V_{12}$ and $W_{12}$ are normal in $I_{12}$ and

$$
1<R_{1} R_{2}<V_{12}<W_{12}<Q_{12}
$$


In addition, we have $Z\left(Q_{1}\right) \cap Z\left(Q_{2}\right)=R_{1} R_{2}, Q_{1} \cap Q_{2}=V_{12}$ is elementary abelian and, setting $\overline{V_{12}}=V_{12} / R_{1} R_{2}$,

$$
\overline{V_{12}}=\overline{V_{1}} \oplus \overline{V_{2}}
$$

where $\overline{V_{1}}$ and $\overline{V_{2}}$ are irreducible $L_{12}$-modules of $\mathrm{GF}(q)$-dimension 4 which are not isomorphic as $\mathrm{GF}(2) L_{12}$-modules. Furthermore, if $q>2, W_{12}^{\prime}=R_{1} R_{2}$ whereas, if $q=2, W_{12}^{\prime}=\left\langle r_{1} r_{2}\right\rangle$ where $r_{i} \in R_{i}^{\#}$.

(iii) $\left[V_{12}, W_{12}\right]=1$ and $W_{12} / V_{12}$ has order $q^{2}$ and is centralized by $L_{12}$.

(iv) We have

$$
Q_{12} / W_{12} \cong Q_{1} W_{12} / W_{12} \oplus Q_{2} W_{12} / W_{12},
$$

$Q_{1} W_{12} / W_{12}$ and $Q_{2} W_{12} / W_{12}$ are irreducible, non-isomorphic $L_{12}$-modules of $\mathrm{GF}(q)$-dimension 4 . Furthermore, as $L_{12}$-modules, for $i=1,2$,

$$
Q_{i} W_{12} / W_{12} \cong V_{3-i} / R_{1} R_{2}
$$

(v) We have

$$
Q_{12} / V_{12}=Q_{1} / V_{12} \oplus Q_{2} / V_{12}
$$

is a direct sum of two indecomposable $L_{12}$-modules of $\mathrm{GF}(q)$ dimension 5 .

(vi) The group $\operatorname{Aut}\left(Q_{12}\right)$ has a subgroup of index 2 which normalizes all of $R_{1}, R_{2}, Q_{1}, Q_{2}, Z\left(Q_{1}\right), Z\left(Q_{2}\right), V_{12}$ and $W_{12}$.

Proof. See [13, Lemma D.8].

Lemma 3.17. Suppose that $X \cong{ }^{2} \mathrm{~F}_{4}(q)$ with $q=2^{2 e+1}, S \in \operatorname{Syl}_{2}(X)$, $R$ is a long root subgroup in $Z(S), P=C_{X}(R)$ and $Q=O_{2}(P)$. Then

(i) $P / Q \cong{ }^{2} \mathrm{~B}_{2}(q)$.

(ii) $R=Z(Q), Z_{2}(Q)$ is elementary abelian and $Z_{2}(Q) / R$ is an irreducible 4-dimensional module for $P / Q$.

(iii) $C_{Q}\left(Z_{2}(Q)\right)$ is non-abelian of order $q^{6}, \Phi\left(C_{Q}\left(Z_{2}(Q)\right)\right)=R$ and $Q / C_{Q}\left(Z_{2}(Q)\right)$ is the natural $P / Q$-module.

(iv) If $q>2$, then $Q / Z_{2}(Q)$ is an indecomposable module.

(v) If $q=2$, then $F^{*}(X)={ }^{2} \mathrm{~F}_{4}(2)^{\prime}$ has index 2 in $X$. We have that $R=Z\left(O_{2}\left(P \cap F^{*}(X)\right)\right), Z_{2}(Q)=Z_{2}\left(Q \cap F^{*}(X)\right)$ and $\left|\left(Q \cap F^{*}(X)\right) / Z_{2}(Q)\right|=16$. Furthermore, $\left(Q \cap F^{*}(X)\right) / Z_{2}(Q)$ and $Z_{2}(Q) / R$ admit $P \cap F^{*}(X)$ irreducibly.

(vi) Let $P_{1}=N_{X}\left(Z_{2}(S)\right)$. Then $P_{1}$ is a maximal parabolic subgroup of $X, P_{1} \neq P, P_{1}$ normalizes $Z_{3}(S)$ which has order 
$q^{3}$ and $P_{1}$ induces $\mathrm{GL}_{2}(q)$ on $Z\left(O_{2}\left(P_{1}\right)\right)=Z_{2}(S)$. Furthermore for $q>2$, we have $W=\left\langle\left(Z_{4}(S) \cap Z_{2}(Q)\right)^{P_{1}}\right\rangle$ is elementary abelian and $W / Z_{3}(S)$ is the natural $\mathrm{GL}_{2}(q)$-module. Further $C_{S}\left(Z_{3}(S)\right) / W$ is an irreducible 4-dimensional module for $\mathrm{SL}_{2}(q)$ and $O_{2}\left(P_{1}\right) / C_{S}\left(Z_{3}(S)\right)$ is the natural $\mathrm{SL}_{2}(q)$-module.

Proof. For the structure of $P$ see [6, Example 3.2.5, page 101] or [4, 12.9]. For part (vi) we refer to [4, 12.9].

Lemma 3.18. Suppose that $X \cong \mathrm{G}_{2}(4), S$ is a Sylow 2-subgroup of $X$ and $R$ is a long root subgroup contained in $Z(S)$. Set $P=N_{X}(R)$, $Q=O_{2}(P)$ and $L=O^{2^{\prime}}(P)$. Then $Z(Q)=R=Q^{\prime}, L / Q \cong \mathrm{SL}_{2}(4) \cong$ Alt(5), $P$ acts irreducibly on $Q / R$ while $L$ induces a direct sum of two natural Alt(5)-modules on $Q / R$. Furthermore, if $R<E \leq Q$ is normalized by $L$, then $E$ is not abelian.

Proof. See [13, Lemma D.10].

Lemma 3.19. Suppose that $X$ is quasisimple and $X / Z(X) \cong{ }^{2} \mathrm{~B}_{2}(8)$. Let $S$ be a Sylow 2-subgroup of $X$. If $Z(X) \neq 1$, then $Z(S)=Z(X)$.

Proof. We may assume that $|Z(X)|=2$. There is an element $\nu$ of order 7 normalizing $S$ such that $[Z(S / Z(X)), \nu]=Z(S / Z(X))$. Let $Y$ be the preimage of $Z(S / Z(X))$. Then $|[Y, \nu]|=8$. Assume $Z(S)>Z(X)$, then $Z(S)=Y$ as $\nu$ normalizes $Z(S)$. Now $[Y, \nu]$ is normal in $S$ and so $S /[Y, \nu]$ is of order 16. Since $C_{S /[Y, \nu]}(\nu)=Y /[Y, \nu]$ and $S /[Y, \nu]$ is not extraspecial, $S /[Y, \nu]$ is elementary abelian. Thus $S=[S, \nu] \times Z(X)$ and Gaschütz's Theorem [8, (I.17.4)] provides a contradiction. Hence $Z(S)=Z(X)$.

In the next lemma we adopt the notation introduced in [6, Table 4.5.1] for inner-diagonal and graph automorphisms of order 2 of groups of Lie type defined over fields of odd characteristic.

Lemma 3.20. Suppose that $p$ is an odd prime and $K$ is quasisimple with $K / Z(K)$ a group of Lie type defined in characteristic $p$. Let $\alpha \in$ $\operatorname{Aut}(K)$ be an automorphism of order 2 . If $E\left(C_{K}(\alpha)\right)=1$, then $C_{K}(\alpha)$ is soluble and one of the following holds where the bold face notation indicates an automorphism which centralizes a Sylow 2-subgroup of $K$.

(i) $K / Z(K) \cong \mathrm{PSL}_{2}\left(p^{e}\right)$ and either $\alpha$ is an inner-diagonal automorphism or $p^{e}=9$ and $\alpha$ is a field automorphism;

(ii) $K \cong \operatorname{PSL}_{3}(3)$ and $\alpha \in\left\{\mathbf{t}_{\mathbf{1}}, \gamma_{1}\right\}$;

(iii) $K \cong \operatorname{PSU}_{3}(3)$ and $\alpha \in\left\{\mathbf{t}_{\mathbf{1}}, \gamma_{1}\right\}$;

(iv) $K / Z(K) \cong \mathrm{PSL}_{4}(3)$ and $\alpha \in\left\{\mathbf{t}_{\mathbf{2}}, \gamma_{2}\right\}$;

(v) $K / Z(K) \cong \mathrm{PSU}_{4}(3)$ and $\alpha \in\left\{\mathbf{t}_{\mathbf{2}}, \gamma_{2}\right\}$; 
(vi) $K / Z(K) \cong \mathrm{PSp}_{4}(3)$ and $\alpha \in\left\{\mathbf{t}_{\mathbf{1}}, t_{2}, t_{2}^{\prime}\right\}$;

(vii) $K / Z(K) \cong \mathrm{P} \Omega_{7}(3)$ and $\alpha=\mathbf{t}_{\mathbf{2}}$;

(viii) $K / Z(K) \cong \mathrm{P} \Omega_{8}^{+}(3)$ and $\alpha=\mathbf{t}_{\mathbf{2}}$;

(ix) $K / Z(K) \cong \mathrm{G}_{2}(3)$ and $\alpha=\mathbf{t}_{\mathbf{1}}$; or

(x) $K \cong{ }^{2} \mathrm{G}_{2}(3)^{\prime}$ and $\alpha=\mathbf{t}_{\mathbf{1}}$.

In particular, in all but case $(i), C_{K}(\alpha)$ is a $\{2,3\}$-group and $F^{*}\left(C_{K}(\alpha)\right)$ is a 2-group.

Proof. If $K / Z(K) \cong \mathrm{PSL}_{2}\left(p^{e}\right)$, then we read the statement from [6, Table 4.5.1 and Proposition 4.9.1 (a) and (e)]. Suppose that $K / Z(K) \not$ $\mathrm{PSL}_{2}\left(p^{e}\right)$. Then [6, Table 4.5.1 and Proposition 4.9.1 (a) and (e)] yields $p^{e}=3$ and that $C_{K}(\alpha)$ can only involve Lie components of type $\mathrm{A}_{1}(3)$ and $\mathrm{D}_{2}(3)$. This observation then leads to the groups listed.

\section{Elementary PROPERTIES of the CONFIGURATiON}

For the convenience of the reader we repeat the most important notions that we presented in the introduction. The group $G$ is a $\mathcal{K}_{2^{-}}$ group, $S$ is a Sylow 2-subgroup of $G$ and $Q$ is a large subgroup of $S$. This means $C_{G}(Q) \leq Q, Q=O_{p}\left(N_{G}(Q)\right)$ and $Q \unlhd N_{G}(A)$ for all $1 \neq A \leq Z(Q)$. We define

$$
\mathcal{L}_{G}(S)=\left\{S \leq L \leq G \mid O_{2}(L) \neq 1, C_{G}\left(O_{2}(L)\right) \leq O_{2}(L)\right\}
$$

and for $L \in \mathcal{L}_{G}(S)$ with $L \not \leq N_{G}(Q)$, set

$$
L^{\circ}=\left\langle Q^{L}\right\rangle \text {. }
$$

Denote by $Y_{L}$ the largest normal elementary abelian subgroup of $L$ such that $O_{2}\left(L / C_{L}\left(Y_{L}\right)\right)=1$ and set $C_{L}=C_{L}\left(Y_{L}\right)$.

Let $\mathfrak{M}_{G}(S)$ be the subset of those $M \in \mathcal{L}_{G}(S)$, for which $C_{M}$ is 2closed, $C_{M} / O_{2}(M) \leq \Phi\left(M / O_{2}(M)\right)$ and $M^{\dagger}=M C_{G}\left(Y_{M}\right)$ is the only maximal element in $\mathcal{L}_{G}(S)$ with $M \leq M^{\dagger}$. In particular, $Y_{M}=Y_{M^{\dagger}}$ by [11, Lemma $1.24(\mathrm{~h})]$.

Suppose that $M \in \mathfrak{M}_{G}(S)$ and $T \in \operatorname{Syl}_{2}\left(C_{G}\left(Y_{M}\right)\right)$. Then $Y_{M}$ is

- tall, if there exists $K$ with $T \leq K \leq G$ such that $O_{2}(K) \neq 1$ and $Y_{M} \not \leq O_{2}(K)$,

- characteristic 2-tall provided there is some $K$ with $T \leq K \leq G$ such that $C_{K}\left(O_{2}(K)\right) \leq O_{2}(K)$ and $Y_{M} \not \leq O_{2}(K)$, and

- asymmetric in $G$, if whenever $g \in G$ and $\left[Y_{M}, Y_{M}^{g}\right] \leq Y_{M} \cap Y_{M}^{g}$, then $\left[Y_{M}, Y_{M}^{g}\right]=1$.

We intend to prove the theorem of this paper by contradiction. Specifically, we work under the following hypothesis. 
Hypothesis 4.1. The group $G$ is a $\mathcal{K}_{2}$-group, $S \in \operatorname{Syl}_{2}(G)$ is contained in at least two maximal 2-local subgroups and $Q \leq S$ is a large subgroup of $G$. Furthermore, there exists $M \in \mathfrak{M}_{G}(S)$ such that $M \not N_{G}(Q)$ and $Y_{M}$ is asymmetric and tall but not characteristic 2-tall.

In this section we collect the rudimentary facts about the configuration of Hypothesis 4.1.

Lemma 4.2. Suppose that $Q \leq K \leq G$ and $O_{2}(K) \neq 1$. Then

(i) $\mathrm{C}_{G}\left(\mathrm{O}_{2}(K)\right)$ is a 2-group;

(ii) $K$ has characteristic 2 ; and

(iii) If, in addition, $O_{2}\left(C_{M}\right) \leq K$, then $Y_{M} \leq O_{2}(K)$.

In particular, $Y_{M} \leq Q$.

Proof. Parts (i) and (ii) are [11, Lemma 1.55 (a)]. Taking $T=S \cap$ $C_{G}\left(Y_{M}\right)=O_{2}(M)$, part (iii) follows from the fact that $Y_{M}$ is not characteristic 2-tall. The final statement follows from (iii) by taking $K=N_{G}(Q)$.

Lemma 4.3. If $1 \neq R \leq O_{2}(M)$ is normalized by $M$, then

$$
M \leq N_{G}(R) \leq M^{\dagger} .
$$

Proof. We have that $M \leq N_{G}(R)$. By assumption $M^{\dagger}$ is the unique maximal element in $\mathcal{L}_{G}(S)$, which contains $M$. As $N_{G}(R) \in \mathcal{L}_{G}(S)$ by Lemma 4.2(ii), $N_{G}(R) \leq M^{\dagger}$.

Recall that if $X$ is a group, $A \leq B \leq X$, then $A$ is weakly closed in $B$ with respect to $X$ provided whenever $x \in X$ and $A^{x} \leq B$, then $A^{x}=A$.

Lemma 4.4. The following hold:

(i) $\mathrm{O}_{2}(M) \in \operatorname{Syl}_{2}\left(C_{G}\left(Y_{M}\right)\right)$;

(ii) $Q$ is weakly closed in $S$ with respect to $G$;

(iii) $\mathrm{O}_{2}(M)$ is weakly closed in $S$ with respect to $G$;

(iv) $Y_{M}=\Omega_{1}\left(Z\left(O_{2}(M)\right)\right) \geq \Omega_{1}(Z(S))$;

(v) $O_{2}\left(\left\langle\mathcal{L}_{G}(S)\right\rangle\right)=1$

(vi) if $N \geq O_{2}(M)$ with $O_{2}(N) \neq 1$ and $N$ has characteristic 2, then $Y_{M} \leq O_{2}(N)$;

(vii) if $U$ is a 2-group which is normalized by $O_{2}(M)$, then $U \leq M^{\dagger}$, in particular, $U$ normalizes $Y_{M}$;

(viii) $M^{\circ}=\left\langle Q^{M^{\circ}}\right\rangle$ and $\left[C_{G}\left(Y_{M}\right), M^{\circ}\right] \leq O_{2}\left(M^{\circ}\right)$.

Proof. The first four parts come from [11, Lemma 2.2(b), (e), (f) and (e) and Lemma 2.6(b)]. 
Part (v) follows from the fact that $\mathcal{L}_{G}(S)$ contains at least two maximal members and part (vi) is a consequence of $Y_{M}$ being noncharacteristic 2-tall and part (i).

For (vii) consider $\mathrm{O}_{2}(M) U$, which is a 2-group. By (iii) we have that $\mathrm{O}_{2}(M)$ is normal in $U O_{2}(M)$ and so $U \leq N_{G}\left(O_{2}(M)\right) \leq M^{\dagger}$. Thus $Y_{M^{\dagger}}$ is normalized by $U$, since $Y_{M}=Y_{M^{\dagger}}$ by definition, $U$ normalizes $Y_{M}$.

Part (viii) follows from [11, Lemmas 1.46 (c) and 1.52 (c)].

We can now formulate the fact that $Y_{M}$ is not characteristic 2-tall, in terms of $O_{2}(M)$ : if $K \geq O_{2}(M)$ with $O_{2}(K) \neq 1$ and $Y_{M} \not \leq O_{2}(K)$, then $F^{*}(K) \neq O_{2}(K)$.

Lemma 4.5. If $X$ is a non-trivial 2-group normalized by $Q$, then $X$ does not centralize $O^{2}\left(M^{\circ}\right)$.

Proof. Suppose that $O^{2}\left(M^{\circ}\right) \leq C_{G}(X)$. As $Q$ normalizes $X$, we have $Z(Q) \cap X \neq 1$ and so $O^{2}\left(M^{\circ}\right) \leq N_{G}(Z(Q) \cap X)$. As $Q$ is large, $O^{2}\left(M^{\circ}\right) \leq N_{G}(Q)$. Therefore,

$$
Q=\left\langle Q^{O^{2}\left(M^{\circ}\right)}\right\rangle=\left\langle Q^{Q O^{2}\left(M^{\circ}\right)}\right\rangle=\left\langle Q^{M^{\circ}}\right\rangle=M^{\circ}
$$

by Lemma 4.4 (viii). Thus $M \leq N_{G}(Q)$, which is a contradiction.

The next lemma plays a very important role in the proof of our theorem.

Lemma 4.6. There exists $y \in Y_{M}^{\#}$ such that $F^{*}\left(C_{G}(y)\right) \neq O_{2}\left(C_{G}(y)\right)$. That is $C_{G}(y)$ does not have characteristic 2. In particular, $M^{\dagger}$ does not act transitively on $Y_{M}^{\#}$.

Proof. The first statement is [11, Theorem F (page 131)]. The remaining part follows as $\Omega_{1}(Z(S)) \cap Y_{M} \neq 1$ and the centralizer of this group has characteristic 2 by Lemma 4.2 (ii).

Lemma 4.7. Suppose that $y \in Y_{M}^{\#}$ and $1 \neq R_{1} \leq R \leq C_{G}(y)$ are 2-groups.

(i) If $Q \leq N_{G}\left(R_{1}\right)$ and $R \leq N_{G}(Q)$, then $N_{G}(R), C_{G}(R), N_{C_{G}(y)}(R)$ and $C_{C_{G}(y)}(R)$ have characteristic 2 .

(ii) If $Z(Q) \cap R \neq 1$, then $N_{G}(R), C_{G}(R), N_{C_{G}(y)}(R)$ and $C_{C_{G}(y)}(R)$ have characteristic 2.

(iii) If $R$ is a non-trivial subgroup of $O_{2}(M)$ which is normalized by $M$, then $N_{C_{G}(y)}(R)$ and $C_{C_{G}(y)}(R)$ have characteristic 2.

(iv) $N_{C_{G}(y)}\left(Y_{M}\right)$ has characteristic 2.

(v) If $z \in Z(Q)^{\#}$ and $J$ is a subnormal subgroup of $C_{G}(y)$, then $C_{J}(z)$ has characteristic 2 . 
(vi) If $z \in Z(Q)^{\#}$ and $K$ is a component of $C_{G}(y)$, then $C_{K}(z)$ has characteristic 2 .

Proof. Suppose the hypotheses of (i) hold. Then, as $R_{1}$ is normalized by $Q$, we have $R_{1} \cap Z(Q) \neq 1$. As $R_{1} \leq R$ also $R \cap Z(Q) \neq 1$. Set $K=N_{G}(R \cap Z(Q))$. Then $Q \leq K$ and $K$ has characteristic 2. As $R$ normalizes $Q$, it also normalizes $R \cap Z(Q)$ and so $R \leq K$. Furthermore $C_{G}(R)=C_{K}(R)$. Now application of Lemma 2.1 (with $C=R \cap Z(Q)$, $X=G$ and $B=R)$ yields $N_{G}(R)$ and $C_{G}(R)$ have characteristic 2 .

Since $y \in N_{G}(R), N_{C_{G}(y)}(R)=C_{N_{G}(R)}(y)$ and $C_{C_{G}(y)}(R)=C_{C_{G}(R)}(y)$ have characteristic 2 by Lemma 2.1 (with $C=1, B=\langle y\rangle$ and $X=$ $N_{G}(R), X=C_{G}(R)$, respectively). This proves (i).

For (ii) take $R_{1}=R \cap Z(Q)$, for (iii) take $R_{1}=R$, and then apply (i).

Part (iv) is a special case of (iii).

For (v), we take $R=R_{1}=\langle z\rangle$ and use (i) to get $C_{C_{G}(y)}(z)$ has characteristic 2. By Lemma 4.2, $Y_{M} \leq Q$ and so $[y, z]=1$. As this property passes to subnormal subgroups by Lemma 2.2. we have $C_{J}(z)$ has characteristic 2. Part (vi) follows from (v).

The next lemma is often used to help conclude that $\left|Y_{M}\right|$ small.

Lemma 4.8. Suppose that $J \leq G$ is normalized by $O_{2}(M)$ and $J$ has characteristic 2. Then $Y_{M} \unlhd O_{2}\left(J Y_{M}\right)$ and $\left\langle Y_{M}^{J}\right\rangle$ is elementary abelian.

Proof. We have $O_{2}(M) J$ has characteristic 2 and $O_{2}(M) \in \operatorname{Syl}_{2}\left(C_{G}\left(Y_{M}\right)\right)$ by Lemma4.4(i). Since $Y_{M}$ is not characteristic 2-tall, $Y_{M} \leq O_{2}\left(O_{2}(M) J\right)$. Hence

$$
Y_{M} \leq O_{2}\left(O_{2}(M) J\right) \cap Y_{M} J \leq O_{2}\left(J Y_{M}\right) .
$$

By Lemma 4.4(vii), $Y_{M}$ is normal in $O_{2}\left(J Y_{M}\right)$ and, as $Y_{M}$ is asymmetric, we also have $\left\langle Y_{M}^{J}\right\rangle$ is elementary abelian.

Define

$$
U_{Q}=\left\langle Y_{M}^{N_{G}(Q)}\right\rangle .
$$

Lemma 4.9. The following hold:

(i) $Y_{M} \leq U_{Q} \leq Q \cap O_{2}(M)$ and $U_{Q}$ is elementary abelian;

(ii) $1 \neq \Omega_{1}(Z(Q)) \cap Y_{M}<Y_{M}$; and

(iii) $Y_{M} \not \leq\left[U_{Q}, Q\right]<U_{Q}$.

Proof. Since $N_{G}(Q) \in \mathcal{L}_{G}(S), Y_{M} \leq Q$ by Lemma 4.2(iii) and $U_{Q}$ is elementary abelian by Lemma 4.8. Thus $U_{Q} \leq C_{Q}\left(Y_{M}\right)=Q \cap C_{S}\left(Y_{M}\right)=$ $Q \cap \mathrm{O}_{2}(M)$ by Lemma 4.4 (i). This is (i).

If $Y_{M} \leq \Omega_{1}(Z(Q))$, then $M \leq M^{\dagger} \leq N_{G}\left(Y_{M}\right) \leq N_{G}(Q)$ as $Q$ is large. This is against the choice of $M$ and so (ii) holds. 
As $Q$ acts on $U_{Q}$, we have $\left[Q, U_{Q}\right]<U_{Q}$. As $\left[Q, U_{Q}\right]$ is normal in $N_{G}(Q)$, we get $Y_{M} \not \subset\left[Q, U_{Q}\right]$, which is (iii).

Lemma 4.10. Assume $N_{G}(Q) \leq M^{\dagger}$. Then there is at least one $L \in$ $\mathcal{L}_{G}(S)$ such that $Y_{L} \not \leq Y_{M}$.

Proof. Assume that for all $L \in \mathcal{L}_{G}(S), Y_{L} \leq Y_{M}$. Then $O_{2}(M) \leq C_{M} \leq$ $C_{L}$. As $O_{2}(M) \leq S \cap C_{L}$ and $O_{2}(M)$ is weakly closed in $S$ by Lemma 4.4, we have that $N_{L}\left(S \cap C_{L}\right) \leq N_{L}\left(O_{2}(M)\right) \leq M^{\dagger}$ by Lemma 4.3. As $C_{L} \leq N_{G}(Q) \leq M^{\dagger}$ by assumption, we get $L=N_{L}\left(S \cap C_{L}\right) C_{L} \leq M^{\dagger}$. Hence $\left\langle\mathcal{L}_{G}(S)\right\rangle \leq M^{\dagger}$ and this contradicts Lemma $4.4(\mathrm{v})$. Thus there exists $L \in \mathcal{L}_{G}(S)$ with $Y_{L} \not \leq Y_{M}$.

We use the previous lemma as follows:

Lemma 4.11. There exists an elementary abelian normal subgroup of $S$ contained in $\mathrm{O}_{2}(M)$ which strictly contains $Y_{M}$. In particular, $Y_{M} \neq \Omega_{1}\left(O_{2}(M)\right), O_{2}(M)$ is not abelian and $Y_{M} \neq J\left(O_{2}(M)\right)$.

Proof. Suppose that $Y_{M}$ is a maximal elementary abelian subgroup of $\mathrm{O}_{2}(M)$, which is normal in $S$. By Lemma 4.9 (i), $Y_{M} \leq U_{Q} \leq$ $\mathrm{O}_{2}(M)$ and $U_{Q}$ is elementary abelian. Thus $U_{Q}=Y_{M}$ and so $N_{G}(Q) \leq$ $M^{\dagger}$. Let $L \in \mathcal{L}_{G}(S)$, then by Lemma 4.2(iii) $Y_{M} \leq O_{2}(L)$ and so $\left[Y_{L}, Y_{M}\right]=1$. Hence $Y_{L} \leq C_{S}\left(Y_{M}\right)=O_{2}(M)$ by Lemma 4.4(i). As $Y_{L}$ is normal in $S$, we have $Y_{L} \leq Y_{M}$ by assumption. Now Lemma 4.10 yields a contradiction. This proves the first claim. Furthermore $Y_{M}<\Omega_{1}\left(O_{2}(M)\right)$.

As $Y_{M} \neq \Omega_{1}\left(O_{2}(M)\right), Y_{M} \neq J\left(O_{2}(M)\right)$. That $O_{2}(M)$ is not abelian, follows as $Y_{M}=\Omega_{1}\left(Z\left(O_{2}(M)\right)\right)$ by Lemma 4.4 (iv).

We finish this section with a look at what happens when $Y_{M}$ has small order.

Lemma 4.12. We have $\left|Y_{M}\right| \geq 16$.

Proof. Assume false. Since $1 \neq \Omega_{1}(Z(Q)) \cap Y_{M} \leq Y_{M}$, Lemma 4.9)(ii) implies $\left|Y_{M}\right|=4$ or 8 . By Lemma 4.6 $M^{\dagger} / C_{M}$ cannot act transitively on $Y_{M}^{\#}$.

If $\left|Y_{M}\right|=4$, then $\left|M^{\dagger} / C_{M}\right|=2$, but by the definition of $Y_{M}=Y_{M^{\dagger}}$ we have that $O_{2}\left(M^{\dagger} / C_{M}\right)=1$, a contradiction.

Thus $\left|Y_{M}\right|=8$. Then $M^{\dagger} / C_{M}$ is a subgroup of $\mathrm{SL}_{3}(2)$ and, as $M^{\dagger}$ does not act transitively on $Y_{M}^{\#}, M^{\dagger} / C_{M}$ is a $\{2,3\}$-group. In particular, $M^{\dagger} / C_{M}$ is soluble. As $O_{2}\left(M^{\dagger} / C_{M}\right)=1$, we have that $M^{\dagger} / C_{M} \cong \operatorname{Sym}(3)$ or is cyclic of order 3 . In both cases there is some $w \in Y_{M}^{\#}$, with $M^{\dagger} \leq C_{G}(w)$. In particular $[w, Q]=1$ and so, as $Q$ is large, $M^{\dagger} \leq C_{G}(w) \leq N_{G}(Q)$, a contradiction. 


\section{The Components of $C_{G}(y)$}

By Lemma 4.6, there is some $y \in Y_{M}^{\#}$ such that $F^{*}\left(C_{G}(y)\right) \neq$ $\mathrm{O}_{2}\left(C_{G}(y)\right)$. In this section we show that we can carefully select $y$ such that $C_{G}(y)$ has a structure which can be used to reach a contradiction in the sections which will follow.

Lemma 5.1. Assume that $z \in Z(Q)$ and $y \in C_{S}(z)$ are involutions. Then $C_{C_{G}(y)}(z)$ has characteristic 2 and $z$ inverts $O\left(C_{G}(y)\right)$. Furthermore, if $K$ is a component of $C_{G}(y)$ which is normalized by $z$, then $K=[K, z]$ and, if $z$ induces an inner automorphism on $K$, then $Z(K)$ is a 2-group.

Proof. By Lemma 4.2(ii), $C_{G}(z)$ has characteristic 2 and therefore so does $C_{C_{G}(y)}(z)$ by Lemma 2.1. In particular $C_{O\left(C_{G}(y)\right)}(z)=1$ and so $z$ inverts $O\left(C_{G}(y)\right)$.

Suppose that $K$ is a component of $C_{G}(y)$ which is normalized by $z$. If $z$ centralizes $K$, then $K$ is a component of $C_{C_{G}(y)}(z)$, a contradiction. Hence $z$ acts non-trivially on $K$ and so $K=[K, z]$. Finally, if $z$ induces as an inner automorphism of $K$, then $K\langle z\rangle=K C_{K\langle z\rangle}(K) \leq$ $C_{G}(Z(K))$. As z inverts $O\left(C_{G}(y)\right)$, we infer that $Z(K)$ is a 2-group.

The next lemma is of fundamental importance.

Lemma 5.2. There exists $y \in Y_{M}^{\#}$ such that $E\left(C_{G}(y)\right) \neq 1$.

Proof. There exists $z \in C_{Y_{M}}(S)^{\#} \subseteq C_{Y_{M}}(Q)^{\#}$ and, for such $z, C_{G}(z)$ has characteristic 2 by Lemma 4.2 (ii). Furthermore, $\left|Y_{M}\right| \geq 16$ by Lemma 4.12 .

Assume that for all $y \in Y_{M}^{\#}, E\left(C_{G}(y)\right)=1$. Then Lemmas 2.3 and 4.6 imply that

$$
\Sigma=\left\langle O\left(C_{G}(b)\right) \mid b \in Y_{M}^{\#}\right\rangle \neq 1
$$

has odd order.

Because $M^{\dagger}$ permutes the elements of $Y_{M}^{\#}, \Sigma$ is normalized by $M^{\dagger}$. Since $C_{M^{\dagger}}(\Sigma)$ is normal in $M^{\dagger}$ and $F^{*}\left(M^{\dagger}\right)$ is a 2-group and is a maximal 2-local subgroup of $G$ implies that $C_{M^{\dagger}}(\Sigma)=1$. In addition, as $\mathrm{O}_{2}\left(C_{G}(z)\right) \leq S \leq M$

$$
\left[C_{\Sigma}(z), O_{2}\left(C_{G}(z)\right)\right] \leq \Sigma \cap O_{2}\left(C_{G}(z)\right)=1
$$

and so $z$ inverts $\Sigma$. Hence $\left[z, M^{\dagger}\right] \leq C_{M^{\dagger}}(\Sigma)=1$ and so $z \in Z\left(M^{\dagger}\right)$. But then $M^{\dagger} \leq C_{G}(z) \leq N_{G}(Q)$, a contradiction.

From now on we focus our interest on the following subset of elements of $Y_{M}$ :

$$
\mathcal{Y}=\left\{y \in Y_{M}^{\#} \underset{24}{E} E\left(C_{G}(y)\right) \neq 1\right\}
$$


which by Lemma 5.2 is non-empty. We also put

$$
\mathcal{Y}_{S}=\left\{y \in \mathcal{Y} \mid C_{S}(y) \in \operatorname{Syl}_{2}\left(C_{G}(y)\right)\right\} \text {. }
$$

From among all the components that appear in $C_{G}(y)$ for $y \in \mathcal{Y}$ select $C$ such that first $|C / Z(C)|$ is maximal and second that $|C|$ is maximal. Then for $y \in \mathcal{Y}$ set

$E_{y}=\langle J| J$ is a component of $C_{G}(y), J / Z(J) \cong C / Z(C)$ and $\left.|J|=|C|\right\rangle$.

Let

$\mathcal{Y}^{*}=\left\{\begin{array}{l|l}y \in \mathcal{Y} & \begin{array}{l}(a) \\ (b)\end{array}\left|E_{y}\right| \text { maximal }\end{array}\right\}$,

and

$$
\mathcal{Y}_{S}^{*}=\mathcal{Y}^{*} \cap \mathcal{Y}_{S}
$$

Lemma 5.3. For $y \in \mathcal{Y}$, there exists $g \in M$ such that $y^{g} \in \mathcal{Y}_{S}$.

Proof. As $O_{2}(M) \leq C_{G}\left(Y_{M}\right) \leq C_{G}(y)$, we may choose $R \in \operatorname{Syl}_{2}\left(C_{G}(y)\right)$ such that $O_{2}(M) \leq R$. Then $R \leq M^{\dagger}$ by Lemma 4.4 (vii). Since $S \in \operatorname{Syl}_{2}\left(M^{\dagger}\right)$, there exists $h \in M^{\dagger}$ such that $R^{h} \leq S$. Hence $R^{h}=$ $C_{S}\left(y^{h}\right) \in \operatorname{Syl}_{2}\left(C_{G}\left(y^{h}\right)\right)$. As $M^{\dagger}=M C_{M^{\dagger}}\left(Y_{M}\right)$ we have $h=g h_{1}$ with $g \in M$ and $h_{1} \in C_{M^{\dagger}}\left(Y_{M}\right)$. Now $y^{h}=y^{g}$. This proves the claim.

By Lemma 5.3 every member of $\mathcal{Y}^{*}$ is conjugate to an element of $\mathcal{Y}_{S}^{*}$, thus $\mathcal{Y}_{S}^{*} \neq \emptyset$.

Lemma 5.4. Suppose that $y \in \mathcal{Y}_{S}$ and $K$ is a component of $E\left(C_{G}(y)\right)$. If $w \in C_{C_{S}(y)}(K)$ is an involution, then $K \leq E\left(C_{G}(w)\right)$. In particular, if $w \in C_{Y_{M}}(K)^{\#}$, then $w \in \mathcal{Y}$.

Proof. Set $X=C_{G}(w)$. Then

$$
K \leq E\left(C_{X}(y)\right) \leq L_{2^{\prime}}\left(C_{X}(y)\right) \leq L_{2^{\prime}}(X)
$$

by $L_{2^{\prime}}$-balance [5, Theorem 5.17]. By Lemma 5.1, $z$ inverts $O\left(L_{2^{\prime}}(X)\right)$ for $z \in \Omega_{1}(Z(S))^{\#}$. Since $z$ centralizes $C_{S}(y) \in \operatorname{Syl}_{2}\left(C_{G}(y)\right)$, $z$ normalizes $K$ and so $[K, z]=K$ by Lemma 5.1. Since $z$ inverts $O\left(L_{2^{\prime}}(X)\right)$, we also have $K=[K, z] \leq C_{X}\left(O\left(L_{2^{\prime}}(X)\right)\right)$. Thus

$$
K \leq C_{L_{2^{\prime}}(X)}\left(O\left(L_{2^{\prime}}(X)\right)\right)=E(X) Z\left(O\left(L_{2^{\prime}}(X)\right)\right) O_{2}\left(L_{2^{\prime}}(X)\right) .
$$

by Lemma 2.4. We conclude that $K \leq E(X)$, as claimed.

Lemma 5.5. Suppose that $y \in \mathcal{Y}_{S}, w \in \mathcal{Y}$ and $K$ is a component in $E_{y}$ which is centralized by $w$. Then either $K$ is a component of $E_{w}$ or $K \leq J_{1} J_{2}$ where $J_{1}$ and $J_{2}=J_{1}^{y}$ are components of $E_{w}, J_{1} / Z\left(J_{1}\right) \cong$ $K / Z(K)$ and $\left|J_{1}\right|=|K|$. In particular, $K \leq E_{w}$. 
Proof. By Lemma 5.4, $K \leq E\left(C_{G}(w)\right)$. Let $J=\left\langle K^{E\left(C_{G}(w)\right)}\right\rangle$. Then $J$ is a product of components of $C_{G}(w)$. By [5, Theorem 5.24 (ii)], $\langle y\rangle$ acts transitively on the components of $C_{G}(w)$ in $J$. If $J$ is a component of $E\left(C_{G}(w)\right)$, then the maximal selection of $K$ implies that $K=J$ and so $K \leq E_{w}$. So suppose that $J=J_{1}^{y} J_{1}$. Then $K \cap J_{1}$ is centralized by $y$ and so $K \cap J_{1} \leq J_{1}^{y} \cap J_{1}$. Thus

$$
K /\left(K \cap J_{1}\right) \cong K J_{1} / J_{1} \leq J_{1}^{y} J_{1} / J_{1} \cong J_{1}^{y} /\left(J_{1}^{y} \cap J_{1}\right)
$$

In particular, the maximal choice of $|K / Z(K)|$ implies that $K / Z(K) \cong$ $J_{1} / Z\left(J_{1}\right)$. Moreover, we calculate

$$
|K|\left|J_{1}^{y} \cap J_{1}\right| \leq\left|J_{1}^{y}\right|\left|K \cap J_{1}\right| \leq\left|J_{1}^{y}\right|\left|J_{1}^{y} \cap J_{1}\right|
$$

and so from the maximal choices of $|K|$ we deduce that $|K|=\left|J_{1}^{y}\right|=$ $\left|J_{1}\right|$. Thus, by definition, $K \leq J \leq E_{w}$, and this completes the proof.

Lemma 5.6. Suppose that $y \in \mathcal{Y}_{S}^{*}$ and $w \in C_{Y_{M}}\left(E_{y}\right)^{\#}$. Then $E_{y}=E_{w}$. In particular, $w \in \mathcal{Y}^{*}$.

Proof. By Lemma 5.4, $w \in \mathcal{Y}$ and then, by Lemma 5.5, $E_{y} \leq E_{w}$. The maximal choice of $\left|E_{y}\right|$ shows $E_{y}=E_{w}$. In particular $w \in \mathcal{Y}^{*}$.

For $y \in \mathcal{Y}_{S}^{*}$, define

$$
\begin{gathered}
S_{y}=C_{S}(y) \cap E_{y} \in \operatorname{Syl}_{2}\left(E_{y}\right) ; \text { and } \\
T_{y}=C_{C_{S}(y)}\left(E_{y}\right) .
\end{gathered}
$$

Observe that Lemma 5.6 implies that $\left(Y_{M} \cap T_{y}\right)^{\#} \subseteq \mathcal{Y}^{*}$.

Lemma 5.7. If $y \in \mathcal{Y}_{S}$ and $F \leq E\left(C_{G}(y)\right)$ is a component of $C_{G}(y)$, then $C_{C_{S}(y)}(F) \cap Z(Q)=1$. In particular, $Z(Q) \cap T_{y}=1$.

Proof. This follows by Lemma 5.1.

Lemma 5.8. Suppose that $y \in \mathcal{Y}_{S}^{*}$ is chosen with $\left|C_{S}(y)\right|$ maximal. Then $C_{S}(y) \in \operatorname{Syl}_{2}\left(N_{G}\left(E_{y}\right)\right)$. In particular, $C_{S}(y)=N_{S}\left(E_{y}\right)$ and $T_{y}=$ $C_{S}\left(E_{y}\right)$

Proof. Plainly $C_{S}(y) \leq N_{G}\left(E_{y}\right)$. Assume that $R \in \operatorname{Syl}_{2}\left(N_{G}\left(E_{y}\right)\right)$ with $R>C_{S}(y)$ and pick $t \in N_{R}\left(C_{S}(y)\right) \backslash C_{S}(y)$. As $t$ normalizes $C_{S}(y) \geq$ $\mathrm{O}_{2}(M)$, Lemma 4.4 (iii) and (iv) imply that $t$ normalizes $Y_{M}$. Hence $\langle t\rangle C_{S}(y)$ normalizes $Y_{M} \cap C_{C_{S}(y)}\left(E_{y}\right) \geq\langle y\rangle$. Thus there exists $w \in$ $\left(Y_{M} \cap C_{C_{S}(y)}\left(E_{y}\right)\right)^{\#}$ which is centralized by $\langle t\rangle C_{S}(y)$. Lemma 5.6 implies $w \in \mathcal{Y}^{*}$ and then the maximal choice of $\left|C_{S}(y)\right|$ together with Lemma 5.3 provide a contradiction. Therefore $C_{S}(y) \in \operatorname{Syl}_{2}\left(N_{G}\left(E_{y}\right)\right)$ and this proves the main claim. It follows at once that $C_{S}(y)=N_{S}\left(E_{y}\right)$ and $C_{S}\left(E_{y}\right)=T_{y}$. 
Lemma 5.9. Let $y \in \mathcal{Y}_{S}^{*}$ with $\left|C_{S}(y)\right|$ maximal. Then $N_{S}\left(T_{y}\right)=C_{S}(y)$.

Proof. Assume the statement is false and choose $t \in N_{S}\left(C_{S}(y)\right) \backslash C_{S}(y)$ with $T_{y}^{t}=T_{y}$. Then $t$ normalizes $U=Z\left(C_{S}(y)\right) \cap T_{y} \cap Y_{M}$. As $y \in U$, $U \neq 1$. Hence there is $1 \neq w \in U$ such that $w^{t}=w$. Since $w \in T_{y}$, $E_{y}=E_{w}$ by Lemma 5.6. But then, by Lemma 5.8, $t \in N_{S}\left(E_{w}\right)=$ $N_{S}\left(E_{y}\right)=C_{S}(y)$, a contradiction.

Suppose that $W$ is a group. A subgroup $H$ of $W$ is called a trivial intersection subgroup in $W$ provided that $H$ is not normal in $W$ and, for all $g \in W \backslash N_{W}(H)$, we have $H \cap H^{g}=1$. The following lemma will play an important role in the proof of our theorem.

Lemma 5.10. Suppose that $y \in \mathcal{Y}_{S}^{*}$ is chosen with $\left|C_{S}(y)\right|$ maximal. Then $T_{y}$ is a trivial intersection subgroup in $S$ and $T_{y} \cap O_{2}(M)$ is a trivial intersection subgroup in $\mathrm{N}_{G}\left(\mathrm{O}_{2}(M)\right)$.

Proof. By Lemma 5.7, $Z(S) \cap T_{y}=1$. Hence $T_{y}$ is not normal in $S$ and also $T_{y} \cap O_{2}(M)$ is not normal in $N_{G}\left(O_{2}(M)\right) \geq S$. Suppose that $g \in$ $N_{G}\left(O_{2}(M)\right)$ and assume $T_{y} \cap T_{y}^{g} \neq 1$. Since $O_{2}(M)=O_{2}(M)^{g}, O_{2}(M)$ normalizes $T_{y} \cap T_{y}^{g}$. Therefore Lemma 4.4(iv) implies $Y_{M} \cap T_{y} \cap T_{y}^{g} \neq 1$. Pick $w \in\left(Y_{M} \cap T_{y} \cap T_{y}^{g}\right)^{\#}$. Then, by Lemma 5.6,

$$
E_{y}=E_{w} .
$$

As $y^{g} \in \mathcal{Y}_{S^{g}}^{*}$, and $w \in T_{y}^{g}$, we also obtain by Lemma 5.6

$$
E_{y^{g}}=E_{w}
$$

and therefore

$$
E_{y}^{g}=E_{y^{g}}=E_{w}=E_{y} .
$$

Hence, as $g \in N_{G}\left(O_{2}(M)\right)$, using Lemma 5.8 for the first and last equality yields

$T_{y}^{g} \cap O_{2}(M)=C_{O_{2}(M)}\left(E_{y}\right)^{g}=C_{O_{2}(M)}\left(E_{y^{g}}\right)=C_{O_{2}(M)}\left(E_{y}\right)=T_{y} \cap O_{2}(M)$.

This proves the $T_{y} \cap O_{2}(M)$ is a trivial intersection subgroup in $N_{G}\left(O_{2}(M)\right)$. If, in fact, $g \in S \leq N_{G}\left(O_{2}(M)\right)$, then, again using Lemma 5.8, we have

$$
T_{y}^{g}=C_{S}\left(E_{y}\right)^{g}=C_{S}\left(E_{y^{g}}\right)=C_{S}\left(E_{y}\right)=T_{y}
$$

which shows that $T_{y}$ is a trivial intersection subgroup in $S$.

Lemma 5.11. Suppose that $y \in \mathcal{Y}_{S}^{*}$ is chosen with $\left|C_{S}(y)\right|$ maximal. Assume that $X \leq Y_{M}$ is normalized by $C_{S}(y) Q$. Then

$$
\left|X \cap T_{y}\right|^{2} \leq|X| \leq\left|X T_{y} / T_{y}\right|^{2} .
$$

In particular, these bounds hold for $X=[Q, y]$ and $X=Y_{M}$. 
Proof. As, by Lemma 4.2(ii), $y \notin Z(Q)$, we can choose $t \in N_{Q}\left(C_{S}(y)\right) \backslash$ $C_{S}(y)$ with $t^{2} \in C_{S}(y)$. If $T_{y}$ is normalized by $t$, then $T_{y} \cap Y_{M} \geq\langle y\rangle$ is normalized by $C_{S}(y)\langle t\rangle$ and so by Lemma 5.6 there exists $w \in \mathcal{Y}^{*}$ with $\left|C_{S}(w)\right| \geq 2\left|C_{S}(y)\right|$, a contradiction. Hence $t \notin N_{S}\left(T_{y}\right)$ and so

$$
\left(T_{y} \cap X\right) \cap\left(T_{y} \cap X\right)^{t} \leq T_{y} \cap T_{y}^{t}=1
$$

by Lemma 5.10. Thus $\left|X \cap T_{y}\right|^{2} \leq|X|$. As

$$
\left|X \cap T_{y}\right|=\left|\left(X \cap T_{y}\right)^{t}\right|=\left|\left(X \cap T_{y}\right)^{t}\left(X \cap T_{y}\right) /\left(X \cap T_{y}\right)\right| \leq\left|X T_{y} / T_{y}\right|,
$$

we also obtain

$$
|X|=\left|X T_{y} / T_{y}\right|\left|\left(X \cap T_{y}\right)\right| \leq\left|X T_{y} / T_{y}\right|^{2} .
$$

Since $Y_{M}$ and $[Q, y] \leq Y_{M}$ are both normalized by $Q C_{S}(y)$, the displayed bounds apply to these subgroups.

Lemma 5.12. Assume that $y \in \mathcal{Y}_{S}^{*}$ and $K$ is a component of $E_{y}$. Suppose that $N_{G}\left(S_{y} T_{y}\right)$ has characteristic 2 . Then $Y_{M} \leq O_{2}\left(N_{G}\left(S_{y} T_{y}\right)\right)$. In particular, if $N_{E_{y}}\left(S_{y}\right)>S_{y} Z\left(E_{y}\right)$, then $Y_{M}$ normalizes $K$.

Proof. We have that $O_{2}(M)$ normalizes $T_{y} S_{y}$. Hence the first assertion follows from Lemma 4.8 .

Let $K$ be a component of $E_{y}$ and $X=S_{y} \cap K$. Then by hypothesis $N_{K}(X)>X Z(K)$. Let $w \in N_{K}(X) \backslash X Z(K)$ have odd order, then $w \in N_{G}\left(T_{y} S_{y}\right)$ and so, for $t \in Y_{M},[w, t] \in O_{2}\left(N_{G}\left(S_{y} T_{y}\right)\right)$. However, if $K \neq K^{t}$, then $w$ and $w^{t}$ commutes and so $[w, t]=w^{-1} w^{t}$ has odd order. We conclude that $Y_{M}$ normalizes $K$.

Definition 5.13. Assume that $W$ is a normal subgroup of a group $X$. Then $W$ has the Sylow centralizer property in $X$ provided that for $T \in \operatorname{Syl}_{2}(X)$ and $R=W \cap T \in \operatorname{Syl}_{2}(W)$,

$$
C_{T C_{X}(W) / C_{X}(W)}\left(R C_{X}(W) / C_{X}(W)\right) \leq W C_{X}(W) / C_{X}(W) .
$$

Lemma 5.14. Assume that $y \in \mathcal{Y}_{S}^{*}$ and that every component $K$ of $E_{y}$ has the Sylow centralizer property in $N_{C_{G}(y)}(K)$. Then $\Omega_{1}(Z(S)) \leq$ $S_{y} T_{y} \in \operatorname{Syl}_{2}\left(E_{y} T_{y}\right)$ and $N_{G}\left(S_{y} T_{y}\right)$ has characteristic 2 .

Proof. Since $\Omega_{1}(Z(S))$ normalizes every component in $E_{y}$ and they each satisfy the Sylow centralizer property in $C_{G}(y)$, we have $\Omega_{1}(Z(S)) \leq$ $S_{y} T_{y}$. The result now follows from Lemma 2.1.

Lemma 5.15. Suppose that $y \in \mathcal{Y}_{S}^{*}$ with $\left|C_{S}(y)\right|$ is maximal and $E_{y}=$ $K$ is quasisimple and satisfies the Sylow centralizer property in $C_{G}(y)$. Assume that $C_{G}(x)$ has characteristic 2 for all $x \in \Omega_{1}\left(Z\left(S_{y}\right)\right) \backslash Z(K)$. Then $T_{y}$ is isomorphic to a subgroup of $Z\left(S_{y}\right) /\left(S_{y} \cap Z(K)\right)$. 
Proof. Lemma 5.7 implies that $S>N_{S}\left(T_{y}\right)$. Let $g \in N_{S}\left(N_{S}\left(T_{y}\right)\right) \backslash$ $N_{S}\left(T_{y}\right)$ with $g^{2} \in N_{S}\left(T_{y}\right)$. Then $T_{y} T_{y}^{g} \leq N_{S}\left(T_{y}\right)=N_{S}\left(T_{y}^{g}\right)$ and, as $T_{y} \neq T_{y}^{g}$, Lemma 5.10 implies

$$
\left[T_{y}, T_{y}^{g}\right] \leq T_{y} \cap T_{y}^{g}=1 .
$$

In particular, as $y \in T_{y}, T_{y}^{g} \leq C_{S}(y)$ and so normalizes $E_{y}=K$ and thus also $S_{y}$. Assume that $T_{y}^{g} \cap S_{y} \neq 1$. Then, as $S_{y}$ normalizes $T_{y}^{g}$, $T_{y}^{g} \cap \Omega_{1}\left(Z\left(S_{y}\right)\right) \neq 1$. By Lemma 5.4 the centralizer of every involution in $T_{y}$ is not of characteristic 2 . The hypothesis on elements of $\Omega_{1}\left(Z\left(S_{y}\right)\right)$ implies that $T_{y}^{g} \cap \Omega_{1}\left(Z\left(S_{y}\right)\right) \leq Z(K) \cap S_{y} \leq C_{C_{S}(y)}(K)=T_{y}$. As $T_{y} \cap T_{y}^{g}=1$, we have a contradiction. Hence $T_{y}^{g} \cap S_{y}=1$. As $T_{y}^{g}$ is normalized by $N_{S}\left(T_{y}\right) \geq S_{y}$, we have $\left[T_{y}^{g}, S_{y}\right] \leq T_{y}^{g} \cap S_{y}=1$. Thus the Sylow centralizer property in $C_{G}(y)$ yields

$$
T_{y}^{g} \leq T_{y} Z\left(S_{y}\right)
$$

As $T_{y} \cap T_{y}^{g}=1$, we conclude that $T_{y}$ is abelian and isomorphic to a subgroup of $Z\left(S_{y}\right) /\left(S_{y} \cap Z(K)\right)=Z\left(S_{y}\right) /\left(S_{y} \cap T_{y}\right)$.

Next, for $y \in \mathcal{Y}$, we study the action of $O_{2}(M)$ and $Y_{M}$ on the components of $C_{G}(y)$.

Lemma 5.16. Assume that $y \in \mathcal{Y}$ and $K$ is a component of $E\left(C_{G}(y)\right)$. If $Y_{M}$ does not normalize $K$, then $K / Z(K)$ has elementary abelian Sylow 2-subgroups.

Proof. We may assume that $y \in \mathcal{Y}_{S}$. Then $Y_{M}$ is an abelian normal subgroup of $C_{S}(y) \in \operatorname{Syl}_{2}\left(C_{G}(y)\right)$ which does not normalize $K$. Hence Lemma 2.5 provides the result.

Lemma 5.17. Suppose that $y \in \mathcal{Y}$ and $K$ is a component of $C_{G}(y)$. If $Z(Q) \cap K \neq 1$, then $F^{*}\left(C_{G}(y)\right)=K O_{2}\left(C_{G}(y)\right)$ and $O\left(C_{G}(y)\right)=1$.

Proof. Since $Z(Q) \cap K \neq 1$, we can select $z \in\left(\Omega_{1}(Z(Q)) \cap K\right)^{\#}$. As $z \in K, z$ centralizes $O\left(C_{G}(y)\right)$ as well as any component $J$ of $C_{G}(y)$ with $J \neq K$. Applying Lemma 5.1 proves the claim.

Lemma 5.18. Suppose that $y \in \mathcal{Y}_{S}$ and $K$ is a component of $E\left(C_{G}(y)\right)$ which is normalized by $Y_{M}$. Assume that $S \cap Z(K)=1$. Then either $S \cap K \leq \mathrm{O}_{2}(M)$ or $\mathrm{O}_{2}(M)$ normalizes $K$.

Proof. If $S \cap K$ centralizes $Y_{M}$, then $S \cap K \leq C_{S}\left(Y_{M}\right)=O_{2}(M)$. Suppose that $Y_{M}$ does not centralize $S \cap K$. Then $1 \neq\left[Y_{M}, S \cap K\right] \leq$ $S \cap K$ and $\left[Y_{M}, S \cap K\right]$ is centralized by $O_{2}(M)$. Thus, for $m \in O_{2}(M)$, $\left[Y_{M}, S \cap K\right] \leq K \cap K^{m}$. If $K \neq K^{m}$ this yields $\left[Y_{M}, S \cap K\right] \leq S \cap Z(K)=$ 1 , a contradiction. Thus $K$ is normalized by $\mathrm{O}_{2}(M)$. 
Lemma 5.19. Suppose that $y \in \mathcal{Y}_{S}$ and $z \in \Omega_{1}(Z(S))^{\#}$. Assume that $K$ is a component of $C_{G}(y)$ and $C_{K}(z)$ is not a 2-group. Then $C_{Q}(y)$ normalizes $K$. In particular, $Y_{M}$ normalizes $K$.

Proof. Assume that the lemma is false. As $z$ inverts $O\left(C_{G}(y)\right)$ by Lemma 5.1, $z$ inverts $O\left(C_{G}(y)\right) \cap Z(K)$ and so, as $C_{K}(z)$ is not a 2-group, there is an odd prime $r$ and $R \in \operatorname{Syl}_{r}\left(C_{K}(z)\right)$ with $R \not \leq Z(K)$. Assume that $C_{Q}(y)$ does not normalize $K$. Then there exists $b \in C_{Q}(y)$ such that $K^{b} \neq K$. Because $K$ is a component of $C_{G}(y),\left[K, K^{b}\right]=1$. Since $C_{G}(z) \leq N_{G}(Q)$ and $b \in Q$, we have

$$
C_{K}(z) Q=\left(C_{K}(z) Q\right)^{b}=C_{K^{b}}(z) Q .
$$

In particular, as $C_{K}(z) C_{K^{b}}(z) \leq C_{K}(z) Q, R \in \operatorname{Syl}_{r}\left(C_{K}(z) C_{K^{b}}(z)\right)$ and so $R R^{b}=R \leq K \cap K^{b} \leq Z(K)$, a contradiction. Hence $C_{Q}(y)$ normalizes $K$. As $Y_{M} \leq C_{Q}(y), Y_{M}$ also normalizes $K$.

Next we show that in many situations $E\left(C_{G}(y)\right)$ is quasisimple.

Lemma 5.20. Suppose that $y \in \mathcal{Y}_{S}, z \in \Omega_{1}(Z(S))^{\#}$ and $K$ is a component of $C_{G}(y)$. Assume there is a non-trivial subgroup $J \leq C_{K}(z)$ such that

(a) $J=O^{2}(J)$ is normalized by $C_{Q}(y)$; and

(b) $[Q, y]$ is centralized by $J$, Then

(i) $Q$ normalizes $J$ and $1 \neq Z(Q) \cap[Q, J] \leq K$; and

(ii) $F^{*}\left(C_{G}(y)\right)=K O_{2}\left(C_{G}(y)\right)$.

In particular, assumption (b) holds if, for all $W \leq Y_{M}$ with $W$ normalized by $J$, we have $[W, J]=1$.

Proof. By (a) and Lemma 5.19, $C_{Q}(y)$ normalizes $K$ and, as $z \in Z(Q)$ and $Q$ is large, $J=O^{2}(J) \leq N_{G}(Q)$ and $[Q, J] \neq 1$. Set $W=[Q, y]$. Then (b) implies $[W, J]=1$ and, as $[J, y]=1$, we have

$$
[Q, J, y]=1
$$

by the Three Subgroups Lemma. In particular, as $J=O^{2}(J)$ and $C_{Q}(y)$ normalizes $C_{K}(z)$ by (a),

$$
[Q, J]=[Q, J, J] \leq\left[C_{Q}(y), J\right] \leq J .
$$

Because $[Q, J] \neq 1$ and $[Q, J]$ is normalized by $Q$, we have that

$$
1 \neq Z(Q) \cap[Q, J] \leq Z(Q) \cap J \leq K .
$$

Thus $Z(Q) \cap K \neq 1$ and so $F^{*}\left(C_{G}(y)\right)=K O_{2}\left(C_{G}(y)\right)$ follows from Lemma 5.17. This proves (i) and (ii).

Now suppose for all $W \leq Y_{M}$ with $W$ normalized by $J$, we have $[W, J]=1$. Then as $[Q, y] \leq Y_{M}$ and is normalized by $J$, (b) holds. 
Lemma 5.21. Suppose that $y \in \mathcal{Y}_{S}$ and $z \in \Omega_{1}(Z(S))^{\#}$. Let $K$ be a component of $C_{G}(y)$ and set $L_{K}=C_{K}(z)$. Assume that $L_{K}$ is not a 2-group. Then $C_{G}\left(Y_{M}\right) C_{Q}(y)$ normalizes $K$. Furthermore, if $Y_{M} \cap K \leq$ $C_{K}\left(O^{2}\left(L_{K}\right)\right)$, then $F^{*}\left(C_{G}(y)\right)=K O_{2}\left(C_{G}(y)\right)$.

Proof. By Lemma 5.19, $Y_{M}$ normalizes $K$. Assume that $Y_{M} \cap K \not \leq$ $Z(K)$. Then, for $m \in C_{G}\left(Y_{M}\right), K^{m} \cap K \geq Y_{M} \cap K$. Hence $K^{m}=K$, and so $C_{G}\left(Y_{M}\right)$ normalizes $K$. Thus the main assertion holds in this case.

If $Y_{M} \cap K \leq Z(K)$, then $Y_{M} \cap K \leq C_{K}\left(O^{2}\left(L_{K}\right)\right)$. Hence suppose that $Y_{M} \cap K \leq C_{K}\left(O^{2}\left(L_{K}\right)\right)$ and set $L_{1}=O^{2}\left(L_{K}\right)$. If $W \leq Y_{M}$ is normalized by $L_{1}$, then, as $W$ normalizes $K$,

$$
\left[W, L_{1}\right] \leq W \cap K \leq Y_{M} \cap K \leq C_{K}\left(L_{1}\right) .
$$

Thus $\left[W, L_{1}\right]=\left[W, L_{1}, L_{1}\right]=1$. Lemma 5.19 now provides the hypothesis for Lemma 5.20 which in turn yields $F^{*}\left(C_{G}(y)\right)=K O_{2}\left(C_{G}(y)\right)$. In particular, $C_{G}\left(Y_{M}\right)$ normalizes $K$. This completes the proof.

Lemma 5.22. Suppose that $z \in \Omega_{1}(Z(S))^{\#}, y \in \mathcal{Y}_{S}$ and $K$ is a component of $C_{G}(y)$. Assume that $J \leq C_{K}\left(Y_{M}\right), O_{2}(M)$ normalizes $J$ and $J$ is not a 2-group. Set $\widetilde{J}=J C_{C_{S}(y)}(K) / C_{C_{S}(y)}(K)$. Then the following hold

(i) $M^{\circ} \leq N_{G}\left(O^{2}(J)\right)$.

(ii) There exist distinct non-central $O^{2}(J)$-chief factors in

$$
O_{2}\left(O^{2}(J)\right) / \Phi\left(O_{2}\left(O^{2}(J)\right)\right)
$$

which are isomorphic as $\mathrm{O}^{2}(J)$-modules. In particular, $\mathrm{O}_{2}(J)$ has at least two non-central $O^{2}(J)$-chief factors.

(iii) $F^{*}\left(C_{G}(y)\right)=K O_{2}\left(C_{G}(y)\right)$ and $O\left(C_{G}(y)\right)=1$.

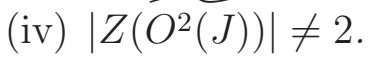

Proof. Lemma 4.4 states $\left[C_{G}\left(Y_{M}\right), M^{\circ}\right] \leq O_{2}(M)$ and so $J_{2}(M)$ is normalized $M^{\circ}$. Hence, as $J$ is normalized by $\mathrm{O}_{2}(M), \mathrm{O}^{2}\left(\mathrm{JO}_{2}(M)\right)=$ $O^{2}(J)$ is normalized by $M^{\circ}$. This is (i).

We have that $O^{2}(J) \neq 1$ by hypothesis. Further, by (i), $Q$ normalizes $O^{2}(J)$. As $O^{2}(J)$ normalizes $Q$, we have $\left[Q, O^{2}(J)\right] \leq Q \cap O^{2}(J)$. As $\left[Q, O^{2}(J)\right] \neq 1$, we have that $Q \cap O^{2}(J) \neq 1$ and so $O_{2}\left(O^{2}(J)\right) \neq 1$.

Assume that (ii) is false. Then the non-central $O^{2}(J)$-chief factors in $\mathrm{O}_{2}\left(O^{2}(J)\right) / \Phi\left(O_{2}\left(O^{2}(J)\right)\right)$ are pairwise non-isomorphic. Since $Q \leq M^{\circ}$ normalizes $O^{2}(J)$ and $O^{2}(J) \leq N_{G}(Q)$, Lemma 2.6 shows that

$$
\left[O_{2}(J), O^{2}(J)\right] / \Phi\left(\left[O_{2}(J), O^{2}(J)\right]\right)
$$


is centralized by $Q$. Since $M^{\circ}$ operates on this factor, we conclude from Burnside's Lemma that $O_{2}\left(O^{2}(J)\right)$ is centralized by $O^{2}\left(M^{\circ}\right)$. This contradicts Lemma 4.5 and completes the proof of (ii).

We have that $\left[Q, O^{2}(J)\right]$ is a non-trivial normal subgroup of $Q$ contained in $K$. It follows that $Z(Q) \cap K \neq 1$. Part (iii) follows from Lemma 5.17.

Suppose that $\left|Z\left(\widetilde{O^{2}(J)}\right)\right|=2$. Observe that $Z(K)$ is a 2-group by (iii). Then, as $O^{2}(J)$ centralizes $Z(K)$,

$$
\left|Z\left(O^{2}(J)\right) Z(K) / Z(K)\right|=\left|Z\left(O^{2}(J)\right): Z\left(O^{2}(J)\right) \cap Z(K)\right| \leq 2 .
$$

By (i), $M^{\circ}$ normalizes $Z\left(O^{2}(J)\right)$. If $Z(K) \cap Z\left(O^{2}(J)\right)=1$, then $M^{\circ}$ centralizes $Z\left(O^{2}(J)\right)$ and this contradicts Lemma 4.5. So assume that $Z(K) \cap Z\left(O_{2}(J)\right) \neq 1$.

As $Z(K) \leq T_{y}$, Lemma 5.7 implies $Z(K) \cap Z(Q)=1$. In particular $Z(K) \cap Z\left(O^{2}(J)\right)$ is not normalized by $Q$ and so there exists $x \in Q$ such that

$$
\left(Z(K) \cap O^{2}(Z(J))\right)\left(Z(K) \cap Z\left(O^{2}(J)\right)\right)^{x}=Z\left(O^{2}(J)\right) .
$$

Since $T_{y}$ is a trivial intersection subgroup in $S$ by Lemma 5.10, we conclude that $Z\left(O^{2}(J)\right)$ has order 4 . As $Z\left(O^{2}(J)\right)$ is normalized by $Q$, we have that $Z\left(O^{2}(J)\right)$ contains elements in $Z(K)^{\#}$ and elements in $Z(Q)^{\#}$. By Lemma 5.4, these elements are not conjugate in $G$, hence $O^{2}\left(M^{\circ}\right)$ centralizes $Z\left(O^{2}(J)\right)$ and again we have a contradiction to Lemma 4.5, This proves (iv).

The next lemma will be used when $K$ is a group of Lie type in characteristic 2 and also for some situations when $K$ is a sporadic simple group. Recall that $U_{Q}$ is defined by $U_{Q}=\left\langle Y_{M}^{N_{G}(Q)}\right\rangle, U_{Q}$ is elementary abelian and $U_{Q} \leq C_{Q}(y)$ for all $y \in Y_{M}$ by Lemma 4.9.

Lemma 5.23. Suppose that $z \in \Omega_{1}(Z(S))^{\#}, y \in \mathcal{Y}_{S}$ and $K$ is a component of $C_{G}(y)$. Set $L_{K}=C_{K}(z)$ and $J_{K}=C_{O^{2}\left(L_{K}\right)}\left(Z\left(O_{2}\left(O^{2}\left(L_{K}\right)\right)\right)\right)$. If $\mathrm{O}_{2}\left(O^{2}\left(L_{K}\right)\right)$ is non-abelian, then $O^{2}\left(J_{K}\right)$ does not act irreducibly on $\mathrm{O}_{2}\left(O^{2}\left(L_{K}\right)\right) / Z\left(O_{2}\left(O^{2}\left(L_{K}\right)\right)\right)$.

Proof. Set $Z=Z\left(O_{2}\left(O^{2}\left(L_{K}\right)\right)\right)$. Then $J_{K}$ centralizes $Z$. By Lemma 4.7 (v), $F^{*}\left(L_{K}\right)=O_{2}\left(L_{K}\right)$. Suppose that

$$
O^{2}\left(J_{K}\right) \text { acts irreducibly on } O_{2}\left(O^{2}\left(L_{K}\right)\right) / Z \text {. }
$$

Since $O_{2}\left(O^{2}\left(L_{K}\right)\right)$ is non-abelian, $O_{2}\left(O^{2}\left(L_{K}\right)\right) / Z$ is not cyclic and so $O^{2}\left(J_{K}\right) \neq 1$. In particular, $O^{2}\left(L_{K}\right) \neq 1$ and so Lemma 5.21 yields

$$
C_{G}\left(Y_{M}\right) C_{Q}(y) \text { normalizes } K \text {. }
$$


As $O^{2}\left(J_{K}\right) \leq L_{K} \leq C_{G}(z) \leq N_{G}(Q), O^{2}\left(J_{K}\right)$ normalizes $U_{Q}=\left\langle Y_{M}^{N_{G}(Q)}\right\rangle$. Using $U_{Q} \leq C_{Q}(y)$ and $C_{Q}(y)$ normalizes $K$, yields $U_{Q}$ normalizes $K$. Furthermore, $U_{Q}$ normalizes $L_{K}=C_{K}(z)$ and therefore also $O^{2}\left(J_{K}\right)$ and so does $C_{Q}(y)$. It follows that

$$
\left[U_{Q}, O^{2}\left(J_{K}\right)\right] \leq U_{Q} \cap O^{2}\left(J_{K}\right) \leq O_{2}\left(O^{2}\left(J_{K}\right)\right) \leq O_{2}\left(O^{2}\left(L_{K}\right)\right) .
$$

Since $\left[U_{Q}, O^{2}\left(J_{K}\right)\right]$ is normalized by $O^{2}\left(J_{K}\right), O^{2}\left(J_{K}\right)$ acts irreducibly on $O_{2}\left(O^{2}\left(L_{K}\right)\right) / Z$ and $O_{2}\left(O^{2}\left(L_{K}\right)\right)$ is non-abelian, but $U_{Q}$ is abelian, we get that $\left[U_{Q}, O^{2}\left(J_{K}\right)\right] \leq Z$. Therefore,

$$
\left[U_{Q}, O^{2}\left(J_{K}\right), O^{2}\left(J_{K}\right)\right] \leq\left[Z, O^{2}\left(J_{K}\right)\right]=1 .
$$

Hence $U_{Q}$ is centralized by $O^{2}\left(J_{K}\right)$ and thus

$$
O^{2}\left(J_{K}\right) \leq C_{G}\left(Y_{M}\right)
$$

Since $C_{G}\left(Y_{M}\right) C_{Q}(y)$ normalizes $K, O_{2}(M)$ normalizes $J_{K}$. As $O_{2}\left(J_{K}\right)$ has exactly one non-central $O^{2}\left(J_{K}\right)$-chief factor, Lemma 5.22 (ii) provides the final contradiction.

Lemma 5.24. Suppose that $z \in \Omega_{1}(Z(S))^{\#}, y \in \mathcal{Y}_{S}$ and $K$ is a component of $C_{G}(y)$. Set $L_{K}=C_{K}(z)$. Assume that $L_{K}$ is not a 2-group, $\mathrm{O}_{2}\left(O^{2}\left(L_{K}\right)\right)$ is elementary abelian and contains exactly one non-central $O^{2}\left(L_{K}\right)$-chief factor. If $Q$ normalizes $O^{2}\left(L_{K}\right)$, then $\left[O_{2}\left(L_{K}\right), O^{2}\left(L_{K}\right)\right] \leq$ $Z(Q)$.

Proof. Since $Q$ normalizes $O^{2}\left(L_{K}\right)$ and $L_{K}$ normalizes $Q,\left[Q, O^{2}\left(L_{K}\right)\right] \leq$ $\mathrm{O}_{2}\left(\mathrm{O}^{2}\left(L_{K}\right)\right)$. The result follows from Lemma 2.6.

Lemma 5.25. Assume that $z \in \Omega_{1}(Z(S))^{\#}, y \in \mathcal{Y}$ and $K$ is a component of $C_{G}(y)$ which is normalized by $Y_{M}$. Then, setting $\widehat{K N_{C_{S}(y)}(K)}=$ $K N_{C_{S}(y)}(K) / C_{C_{S}(y)}(K)$, we have

$$
\left|\widetilde{Y_{M}}\right| \geq\left|\widetilde{\left\langle z^{M}\right\rangle}\right|>2
$$

Proof. Let $K$ be a component of $C_{G}(y)$ and assume that

$$
\widetilde{\left\langle z^{M}\right\rangle} \mid \leq 2
$$

Then, for all $m \in M, z^{m} \in Y_{M}^{\#}$ and Lemma 5.1 implies $z^{m}$ acts nontrivially on $K$. Therefore

$$
\widetilde{z}=\widetilde{z^{m}}
$$

for all $m \in M$. Hence

$$
[z, m]=z^{-1} z^{m} \in C_{C_{S}(y)}(K)
$$

for all $m \in M$. Therefore $[\langle z\rangle, M] \leq C_{C_{S}(y)}(K)$. If $[\langle z\rangle, M] \neq 1$, then $K$ is a component of $N_{C_{G}(y)}([\langle z\rangle, M])$ and this contradicts Lemma 4.7(iii). 
Hence $M \leq C_{G}(z) \leq N_{G}(Q)$ and this is a contradiction to our basic assumption.

Lemma 5.26. Suppose that $z \in \Omega_{1}(Z(S))^{\#}$ and $y \in \mathcal{Y}_{S}^{*}$. Let $K$ be a component of $C_{G}(y)$ and $L_{K}=C_{K}(z)$. Assume $L_{K} / Z\left(L_{K}\right)$ is not a 2-group and set $K \widehat{N_{C_{S}(y)}}(K)=K N_{C_{S}(y)}(K) / C_{C_{S}(y)}(K)$.

(i) If $\left|\widetilde{Y_{M}}\right| \leq 4$, then $F^{*}\left(C_{G}(y)\right)=K O_{2}\left(C_{G}(y)\right)$ and $O^{2}\left(L_{K}\right)$ is normalized by $Q$.

(ii) If $\left|\Omega_{1}\left(C_{K N_{C_{S}(y)}(K)}\left(O^{2}\left(\widetilde{L_{K}}\right)\right)\right) \cap \widetilde{Y_{M}}\right|=2$, then $\left|\widetilde{Y_{M}}\right| \geq 8$.

Proof. Suppose that $\left|\widetilde{Y_{M}}\right| \leq 4$. Assume that $W \leq Y_{M}$ is normalized by $O^{2}\left(L_{K}\right)$. If $|\widetilde{W}|=2$, then $O^{2}\left(L_{K}\right)$ centralizes $W$ and, if $|\widetilde{W}|=4$, then, as $O^{2}\left(L_{K}\right)$ centralizes $\widetilde{\langle z\rangle}$, again $O^{2}\left(L_{K}\right)$ centralizes $W$. Lemma 5.19 implies Lemma 5.20(a) holds. Hence Lemma 5.20 yields $K=E\left(C_{G}(y)\right)$ and $O^{2}\left(L_{K}\right)$ is normalized by $Q$. In particular (i) holds.

To prove (ii), assume that $\left|\widetilde{Y_{M}}\right| \leq 4$ and set $X=C_{Y_{M}}\left(O^{2}\left(L_{K}\right)\right)$. Then, by (i), $K=E\left(C_{G}(y)\right), X$ is normalized by $Q$ and it is also normalized by $C_{S}(y)$. In particular, $C_{C_{S}(y)}(K)=T_{y}$. Since $X$ is elementary abelian, $\left|X T_{y} / T_{y}\right| \leq 2$ holds by assumption. Using Lemma 5.11 yields $|X| \leq 4$. As $\widetilde{z} \in \widetilde{X}$ and $y \in C_{Y_{M}}(K) \leq X$, we deduce that $|X|=4$ and $\left|C_{Y_{M}}(K)\right|=2$. Hence $\left|Y_{M}\right|=\left|\widetilde{Y_{M}}\right|\left|C_{Y_{M}}(K)\right| \leq 8$ and this contradicts Lemma 4.12. Therefore (ii) holds.

Lemma 5.27. Suppose that $y \in Y_{M}^{\#}$ and $K$ is a component of $C_{G}(y)$. Let $P$ be a 2-local subgroup of $K$, and assume that both $K$ and $P$ are normalized by $\mathrm{O}_{2}(M)$. Set $K \widehat{N_{C_{S}(y)}}(K)=K N_{C_{S}(y)}(K) / C_{C_{S}(y)}(K)$. If $P$ is of characteristic 2 , then $\widetilde{Y_{M}} \leq \mathrm{O}_{2}\left(\widetilde{\mathrm{PO}_{2}(M)}\right)$.

Proof. Set $H=P_{2}(M) C_{C_{S}(y)}(K) \leq C_{G}(y)$. Then $O^{2}\left(C_{H}\left(O_{2}(H)\right)\right) \leq$ $P$ and so $O^{2}\left(C_{H}\left(O_{2}(H)\right)\right) \leq C_{P}\left(O_{2}(P)\right) \leq O_{2}(P)$, as $P$ has characteristic 2. Hence $H$ has characteristic 2 and so Lemma 4.8 gives $Y_{M} \leq O_{2}(H)$. Therefore $\widetilde{Y_{M}} \leq O_{2}(\widetilde{H})$.

\section{The STANDARD SETUP AND CONSOLIDATION OF NOTATION}

Throughout the remainder of this paper Hypothesis 4.1 holds. We pick and fix $y \in \mathcal{Y}_{S}^{*}$ with $\left|C_{S}(y)\right|$ maximal. We continue the notation

$$
S_{y}=C_{S}(y) \cap E_{y} \text { and } T_{y}=C_{C_{S}(y)}\left(E_{y}\right)
$$

where $E_{y}$ is as defined just before Lemma 5.4. Recall that $C_{S}(y)$ is a Sylow 2-subgroup of $C_{G}(y)$ by the definition of $\mathcal{Y}_{S}$ and so $S_{y}$ is a Sylow 2-subgroup of $E_{y}$. Furthermore by Lemma 5.8 we have that $C_{S}\left(E_{y}\right)=$ 
$C_{C_{S}(y)}\left(E_{y}\right)$. The subgroup $K$ represents an arbitrary component of $E_{y}$. We denote by $\sim$ the projection

$$
\sim: K N_{C_{S}(y)}(K) \rightarrow K N_{C_{S}(y)}(K) / C_{C_{S}(y)}(K) .
$$

Thus $\widetilde{K}=K C_{C_{S}(y)}(K) / C_{C_{S}(y)}(K) \cong K / Z(K)$. By Lemma4.11, $O_{2}(M)^{\prime} \neq$ 1 and, by Lemma 4.4(iv), $Y_{M}=\Omega_{1}\left(Z\left(O_{2}(M)\right)\right)$. Hence we will fix an involution

$$
z \in \Omega_{1}(Z(S)) \cap Y_{M} \cap O_{2}(M)^{\prime} \leq Z(Q) .
$$

Since $z$ centralizes $C_{S}(y)$ and so $S_{y}, z$ normalizes $K$. We know from Lemma 5.1 that $K=[K, z]$ and $z$ inverts $O\left(C_{G}(y)\right)$. We set

$$
L_{K}=C_{K}(z) .
$$

Obviously, $L_{K} \leq C_{G}(z) \leq N_{G}(Q)$ and so $[Q, y] \leq Y_{M}$ is normalized by $L_{K}$. Furthermore, if $L_{K}$ is not a 2-group, Lemma 5.21 implies that $C_{G}\left(Y_{M}\right) C_{Q}(y)$ normalizes $K$ and, in particular, $O_{2}(M)$ normalizes $L_{K}$. We will often require the subgroup

$$
U_{Q}=\left\langle Y_{M}^{N_{G}(Q)}\right\rangle
$$

which is elementary abelian and contained in $Q \cap O_{2}(M)$ by Lemma 4.9 ,

The next five sections investigate the various possibilities for the isomorphism type of $K / Z(K)$.

\section{SPORADIC GROUPS AS COMPONENTS}

The aim of this section is to show that $K / Z(K)$ cannot be a sporadic simple group or the Tits group ${ }^{2} \mathrm{~F}_{4}(2)^{\prime}$. We begin with $\mathrm{Ru}$ and ${ }^{2} \mathrm{~F}_{4}(2)^{\prime}$.

Lemma 7.1. $K / Z(K) \neq^{2} \mathrm{~F}_{4}(2)^{\prime}$ or $\mathrm{Ru}$.

Proof. We first provide some structural detail about the groups $X^{*} \cong$ ${ }^{2} \mathrm{~F}_{4}(2)^{\prime},{ }^{2} \mathrm{~F}_{4}(2)$ and $\mathrm{Ru}$. Suppose that $x$ is a 2-central involution in $X^{*}$. Then by Lemma 3.17 and [16, page 65] the centralizer $X=C_{X^{*}}(x)$ has the following normal subgroup structure:

$$
1 \leq X_{1}<X_{2} \leq X_{3}<X_{4}=O_{2}(X)<X
$$

where $\left|X_{1}\right|=2, X_{1}=Z\left(X_{4}\right), X_{2}$ is elementary abelian of order 32, $X_{3}=C_{X}\left(X_{2}\right), X_{2}=\Omega_{1}\left(X_{3}\right)$ and $O^{2}(X)$ acts irreducibly on $X_{2} / X_{1}$ and on $X_{4} / X_{3}$ each of order 16. Furthermore,

$$
X_{3} \cong \begin{cases}X_{2} & X^{*} \cong{ }^{2} \mathrm{~F}_{4}(2)^{\prime} \\ 4 \times 2^{4} & X^{*} \cong{ }^{2} \mathrm{~F}_{4}(2) \\ \mathrm{Q}_{8} \times 2^{4} & X^{*} \cong \mathrm{Ru} .\end{cases}
$$


Finally, if $X^{*} \cong{ }^{2} \mathrm{~F}_{4}(2)$ or ${ }^{2} \mathrm{~F}_{4}(2)^{\prime}$, then $X / X_{4} \cong{ }^{2} \mathrm{~B}_{2}(2) \cong \operatorname{Frob}(20)$, while, if $X^{*} \cong \mathrm{Ru}$, then $X / X_{4} \cong \operatorname{Sym}(5)$.

Recall the Sylow centralizer property from Definition 5.13, By 6. Table 5.3r], Aut $(\mathrm{Ru})=\mathrm{Ru}$ and so, when $K / Z(K) \cong \mathrm{Ru}$, the Sylow centralizer property holds for $K$ in $N_{C_{G}(y)}(K)$. We read from [6, Theorem 2.5.12 and Theorem 2.5.15] that $\operatorname{Aut}\left({ }^{2} \mathrm{~F}_{4}(2)^{\prime}\right)={ }^{2} \mathrm{~F}_{4}(2)=$ Aut $\left({ }^{2} \mathrm{~F}_{4}(2)\right)$. As presented above for $X^{*} \cong{ }^{2} \mathrm{~F}_{4}(2)$, we have that $X_{1}=$ $Z\left(X_{4}\right)=Z(S)$. Thus the Sylow centralizer property also holds when $K / Z(K) \cong{ }^{2} \mathrm{~F}_{4}(2)^{\prime}$.

Suppose $K / Z(K) \cong{ }^{2} \mathrm{~F}_{4}(2)^{\prime}$ or Ru. Notice that either $Z(K)=1$ or $K \cong 2 \cdot \mathrm{Ru}$. As $L_{K} \geq S_{y}, L_{K}$ projects $\bmod Z(K)$ onto $X$ as described above (in the cases $X^{*} \cong \mathrm{Ru}$ and $\left.X^{*} \cong{ }^{2} \mathrm{~F}_{4}(2)^{\prime}\right)$. For $1 \leq i \leq 4$, we define $B_{i} \leq L_{K}$ to be the preimage of the subgroup $X_{i}$. Since $L_{K}$ is not a 2-group, Lemma 5.21 implies that $K$ is normalized by $C_{G}\left(Y_{M}\right) C_{Q}(y)$. We have

$$
\widetilde{K O_{2}(M)} \cong \mathrm{Ru},{ }^{2} \mathrm{~F}_{4}(2)^{\prime} \text { or }{ }^{2} \mathrm{~F}_{4}(2)
$$

and, as $C_{K N_{C_{S}(y)}(K)}\left(\widetilde{O^{2}\left(L_{K}\right)}\right)=\widetilde{B_{1}}$ has order 2, Lemma 5.26 (ii) implies that

$$
\left|\widetilde{Y_{M}}\right| \geq 8
$$

Suppose that $W \leq Y_{M}$ is normalized but not centralized by $O^{2}\left(L_{K}\right)$. Then $\widetilde{B_{2}} \leq \widetilde{W} \leq \widetilde{Y_{M}}$ and so $\left|\widetilde{Y_{M}}\right| \geq 2^{5}$. Hence, as $X_{2}=\Omega_{1}\left(C_{X}\left(X_{2}\right)\right)$,

$$
\widetilde{Y_{M}}=\widetilde{B}_{2} \text {. }
$$

Now we have

$$
\Omega_{1}\left(C_{O_{2}(\widetilde{M)(S \cap K)}}\left(\widetilde{Y_{M}}\right)\right)=\Omega_{1}\left(\widetilde{B}_{3}\right)=\widetilde{B}_{2}=\widetilde{Y_{M}} .
$$

It follows that $\Omega_{1}\left(O_{2}(M)\right) C_{C_{S}(y)}(K)=Y_{M} C_{C_{S}(y)}(K)$, which means that $\left[\Omega_{1}\left(O_{2}(M)\right), O_{2}(M)\right] \leq C_{C_{S}(y)}(K)$. Since $K$ does not centralize any element of $Z(Q)$ by Lemma 5.7, we have $\Omega_{1}\left(O_{2}(M)\right)=\Omega_{1}\left(Z\left(O_{2}(M)\right)\right)=$ $Y_{M}$ and this contradicts Lemma 4.11, Therefore

$O^{2}\left(L_{K}\right)$ centralizes every subgroup of $Y_{M}$ which it normalizes.

By Lemma 5.20

$$
F^{*}\left(C_{G}(y)\right)=K O_{2}\left(C_{G}(y)\right) \text { and } Q \text { normalizes } O^{2}\left(L_{K}\right) .
$$

Select $g \in N_{Q}\left(C_{S}(y)\right) \backslash C_{S}(y)$. We have $T_{y} T_{y}^{g}$ is centralized by $K \cap K^{g}$ and $K \cap K^{g} \geq O^{2}\left(L_{K}\right)$ as $Q$ normalizes $O^{2}\left(L_{K}\right)$. Hence

$$
\widetilde{T_{y}^{g}} \leq \widetilde{C_{K C_{S}(y)}}\left(O^{2}\left(\widetilde{L_{K}}\right)\right)=\widetilde{B_{1}}
$$

which has order 2. As $T_{y}^{g} \cap T_{y}=1$ by Lemma 5.10 and Lemma [5.9, we conclude that $\left|T_{y}\right|=2$. 
Suppose that $K / Z(K) \cong \mathrm{Ru}$. Then by [6, Table $5.3 \mathrm{r}]$ there is a 2-local subgroup $J$ of $K$ containing $S_{y}$ with

$$
J / Z(K) \sim 2^{3+8} \cdot \mathrm{SL}_{3}(2)
$$

and $J$ is normalized by $O_{2}(M)$. Hence Lemma 4.8 implies that $Y_{M} \leq$ $O_{2}\left(J Y_{M}\right)$ and $\left\langle Y_{M}^{J}\right\rangle$ is an elementary abelian. Now the structure of $\bar{J}$ and the fact that $O_{2}(J / Z(K))$ is non-abelian implies that $\widetilde{\left\langle Y_{M}^{J}\right\rangle} \mid=2^{3}$. Hence $\widetilde{\left\langle Y_{M}^{J}\right\rangle}=\widetilde{Y_{M}}$ and $\widetilde{O_{2}(M)} \leq O_{2}(\widetilde{J})$. Thus $O_{2}(M) \leq O_{2}\left(O_{2}(M) J\right)$ and Lemma 4.4 implies $J$ normalizes $O_{2}(M)$ and so also $Y_{M}$. Lemma 4.3 yields $J \leq M^{\dagger}$ and $J$ induces $\mathrm{SL}_{3}(2)$ on $Y_{M} /\langle y\rangle$. As $M^{\dagger}$ does not act transitively on $Y_{M}^{\#}$ and $O_{2}\left(M^{\dagger} / C_{M}\right)=1$, the subgroup structure of $\mathrm{SL}_{4}(2)$ yields $M^{\dagger}=J C_{M}$. But then $C_{Y_{M}}\left(M^{\dagger}\right)=\langle y\rangle$, a contradiction as $y \notin Z(S)$. Hence $K / Z(K) \approx \mathrm{Ru}$.

Suppose that $K \cong{ }^{2} \mathrm{~F}_{4}(2)^{\prime}$. As $T_{y}=\langle y\rangle, Y_{M} K=T_{y} K=\langle y\rangle \times K$ and so $Y_{M} \cap K$ has index 2 in $Y_{M}$. Since $F^{*}\left(C_{G}(y)\right)=K O_{2}\left(C_{G}(y)\right)=$ $K\langle y\rangle$, we have $C_{G}(y)=K C_{S}(y)$. Because $L_{K}$ normalizes $Q, L_{K}$ normalizes $U_{Q}=\left\langle Y_{M}^{N_{G}(Q)}\right\rangle$ which is elementary abelian. Again we have $U_{Q} K=\langle y\rangle \times K$ and so $U_{Q} \cap K$ is an elementary abelian subgroup of $K$ normalized by $L_{K}$. We deduce that $K \cap U_{Q}=B_{2} \geq Y_{M} \cap K$. Since $Y_{M} \cap K=C_{B_{2}}\left(O_{2}(M)\right), O_{2}(M) L_{K} / O_{2}\left(O_{2}(M) L_{K}\right) \cong \operatorname{Frob}(20)$ and $B_{2} / Z\left(O_{2}\left(L_{K}\right)\right)$ is an irreducible 4-dimensional $L_{K}$-module, either $O_{2}(M) \leq O_{2}\left(O_{2}(M) L_{K}\right)$ or $\left|Y_{M} \cap K\right|=8$. In the former case $L_{K} \leq M^{\dagger}$ as $O_{2}(M)$ is weakly closed in $S$. But then $L_{K}$ normalizes $Y_{M}$ and this contradicts $O^{2}\left(L_{K}\right)$ centralizing every subgroup of $Y_{M}$ that it normalizes. Hence

$$
\left|Y_{M} \cap K\right|=8 \text { and }\left|Y_{M}\right|=16 .
$$

By Lemma 3.17(iv), $\left|Z_{2}\left(S_{y}\right)\right|=4$ and so $Z_{2}\left(S_{y}\right) \leq Y_{M}$. In particular, $P_{1}=N_{K}\left(Z_{2}\left(S_{y}\right)\right)$ normalizes $C_{O_{2}(M) K}\left(Z_{2}\left(S_{y}\right)\right) \geq O_{2}(M)$ and so $O_{2}(M)$ and $Y_{M}$ are normalized by $P_{1}$.

Since $O_{2}(M)^{\prime} \neq 1$, we have $O_{2}(M)^{\prime} \cap Y_{M} \neq 1$. As $O_{2}(M)^{\prime} \leq K$, we have $Y_{M} \cap O_{2}(M)^{\prime}$ is either $Y_{M} \cap K$ or $Z_{2}\left(S_{y}\right)$. If $Y_{M} \cap O_{2}(M)^{\prime}=Z_{2}\left(S_{y}\right)$, then $M C_{M} / C_{M}$ embeds into the stabilizer of a 2-space in $\mathrm{SL}_{4}(2)$, which is isomorphic to $2^{4} \cdot(\operatorname{Sym}(3) \times \operatorname{Sym}(3))$. Since $P_{1} / C_{P_{1}}\left(Y_{M} \cap K\right) \cong$ $\operatorname{Sym}(4)$, this means that $O_{2}\left(M C_{M} / C_{M}\right) \neq 1$, a contradiction. Therefore $M$ normalizes $Y_{M} \cap K=Y_{M} \cap O_{2}(M)^{\prime}$ and, as $O_{2}\left(M C_{M} / C_{M}\right)=$ 1 and $M C_{M} / C_{M}$ is isomorphic to a subgroup $\mathrm{SL}_{3}(2)$, again using $P_{1} / C_{P_{1}}\left(Y_{M} \cap K\right) \cong \operatorname{Sym}(4)$ yields $M C_{M} / C_{M} \cong \operatorname{SL}_{3}(2)$. Now $y^{M}$ has size 1,7 or 8 . In the first two cases $y$ is centralized by a conjugate of $S$, a contradiction. In the latter case, $y$ is centralized by an element of order 7 in $M$ and this contradicts the fact that $|K|$ is coprime to 7 . Hence $K \approx^{2} \mathrm{~F}_{4}(2)^{\prime}$. 
Proposition 7.2. $K / Z(K)$ is not a sporadic simple group.

Proof. We use the information from [6, Table 5.3] to see that $K$ satisfies the Sylow centralizer property Definition 5.13 in $N_{C_{G}(y)}(K)$. Hence $z$ induces a 2-central involution on $K$. By Lemma 4.7 (v), $F^{*}\left(L_{K}\right)$ is a 2group and, in particular, $L_{K}$ does not have a component. It follows that $K / Z(K)$ is not $\mathrm{J}_{1}, \mathrm{Co}_{3}, \mathrm{McL}$, LyS, O'N or M(23). By Lemma 5.23, if $O_{2}\left(O^{2}\left(L_{K} / Z(K)\right)\right)$ has derived group and Frattini subgroup of order 2, then $O^{2}\left(L_{K}\right)$ does not act irreducibly on $O_{2}\left(O^{2}\left(L_{K}\right)\right) / Z\left(O_{2}\left(O^{2}\left(L_{K}\right)\right)\right)$. Using [6, Table 5.3] shows that $K / Z(K) \neq \operatorname{Mat}(11), \mathrm{J}_{2}, \mathrm{~J}_{3}, \mathrm{~J}_{4}, \mathrm{Co}_{1}$, $\mathrm{Co}_{2}$, Suz, M(22), M(24)', $\mathrm{F}_{1}, \mathrm{~F}_{2}, \mathrm{~F}_{3}$, or $\mathrm{F}_{5}$. Because of Lemma 7.1 the groups which remain to be considered are

$$
K / Z(K) \cong \operatorname{Mat}(12), \operatorname{Mat}(22), \operatorname{Mat}(23), \operatorname{Mat}(24), \text { HS, and He. }
$$

Using [6, Table 5.3] we observe that $L_{K}$ is not a 2-group. In particular, $C_{G}\left(Y_{M}\right) C_{Q}(y)$ normalizes $K$ by Lemma 5.21 .

(7.2.1) Either $\left|\widetilde{Y_{M}}\right| \geq 8$ or $K / Z(K) \cong \operatorname{Mat}(22)$ and $\widetilde{Y_{M}} \not \widetilde{K}$.

Using [6, Table 5.3] we see that $C_{K N_{C_{S}(y)}(K)}\left(\widetilde{O^{2}\left(L_{K}\right)}\right)$ has order 2 unless $K / Z(K) \cong \operatorname{Mat}(22)$ in which case it has order 4 and is not contained in $\widetilde{K}$. Hence Lemma 5.26 gives the result.

Suppose that $K / Z(K) \cong$ HS. Then $L_{K} / Z(K)$ has shape $\left(2_{+}^{1+4}\right.$ 。 4). $\operatorname{Sym}(5)$. As $L_{K} \leq N_{G}(Q)$ and $\left\langle Y_{M}^{L_{K}}\right\rangle \leq U_{Q} \cap O_{2}\left(L_{K}\right) Y_{M}$ is elementary abelian, we obtain from [6. Table $5.3 \mathrm{~m}$ ] that $Y_{M}$ projects into $\Omega_{1}\left(Z\left(L_{K} / Z(K)\right)\right)$. Thus $\left|Y_{M} / C_{Y_{M}}(K)\right|=2$, contrary to (7.2.1)

Assume that $K / Z(K)$ is one of Mat(22), Mat(23), Mat(24) or He. Let $J \in \mathcal{L}_{K}(S \cap K)$ be normalized by $O_{2}(M)$. Then $Y_{M} \leq O_{2}\left(J Y_{M}\right)$ and $\left\langle Y_{M}^{J}\right\rangle$ is elementary abelian by Lemma 4.8. Hence

(7.2.2) $Y_{M} \leq \bigcap_{\substack{J \in \mathcal{L}_{K}(S \cap K) ; \\ O_{2}(M) \leq N_{G}(J)}} O_{2}\left(J Y_{M}\right)$.

Assume that $K \cong$ He or $\operatorname{Mat}(24)$. Then $S \cap K$ is isomorphic to a Sylow 2-subgroup of $\mathrm{SL}_{5}(2)$. Hence $S \cap K$ has exactly two elementary abelian subgroups $E_{1}, E_{2}$ of order 64 and they intersect in a group of order $2^{4}$. Also note that $O_{2}\left(L_{K}\right)$ is the unique extraspecial subgroup of order $2^{7}$ in $S \cap K$. For $i=1,2$, set $J_{i}=N_{K}\left(E_{i}\right)$. If $J_{1}$ and $J_{2}$ are conjugate in $K O_{2}(M)$, then $\widetilde{K O_{2}(M)} \cong \operatorname{Aut}(\mathrm{He})$ and $L_{K} O_{2}(M)$ acts irreducibly on $O_{2}\left(L_{K}\right) / Z\left(O_{2}\left(L_{K}\right)\right)$. Hence $O_{2}\left(L_{K}\right) \leq\left\langle Y_{M}^{L_{K} O_{2}(M)}\right\rangle \leq U_{Q}$ which is a contradiction as $U_{Q}$ is abelian. Therefore $O_{2}(M)$ normalizes 
$J_{1}$ and $J_{2}$, and, as $J_{1}$ and $J_{2}$ have characteristic 2 , we get by (7.2.2)

$$
\widetilde{Y_{M}} \leq \widetilde{E_{1}} \cap \widetilde{E_{2}} \cap \widetilde{O_{2}\left(L_{K}\right)}=Z_{2} \widetilde{(S \cap K)} .
$$

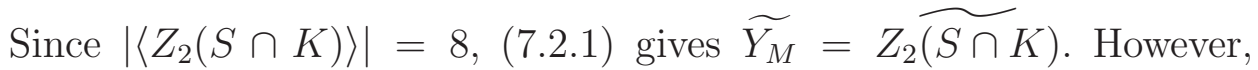
$\left\langle Z_{2} \widetilde{(S \cap K)} \widetilde{L}_{K}\right\rangle=\widetilde{O_{2}\left(L_{K}\right)}$ which is not abelian whereas $\left\langle{\widetilde{Y_{M}}}^{\widetilde{L}_{K}}\right\rangle \leq \widetilde{U}_{Q}$ which is abelian. As this is impossible, we conclude $K / Z(K) \neq \operatorname{Mat}(24)$ or He.

Assume next that $K / Z(K) \cong \operatorname{Mat}(22)$ or Mat(23). Then from [6, Table 5.3c and 5.3d], $(S \cap K) / Z(K)$ has two elementary abelian subgroups $E_{1} / Z(K), E_{2} / Z(K)$ of order 16 with normalizers in $K$ that are of characteristic 2 , where $N_{K}\left(E_{1} / Z(K)\right) \cong 2^{4} \cdot \operatorname{Sym}(5)$ and $N_{K}\left(E_{2} / Z(K)\right) \cong$ $2^{4}$.Alt(6), $2^{4}$.Alt(7), respectively. Furthermore, they are normalized by $O_{2}(M)$. We have $O_{2}\left(\widetilde{N_{K O_{2}(M)}}\left(E_{2}\right)\right) \leq \widetilde{C_{O_{2}(M)}}\left(\widetilde{E_{2}}\right)=\widetilde{E_{2}} \leq \widetilde{K}$ and thus by $(7.2 .2)$

$$
\widetilde{Y_{M}} \leq \widetilde{E_{1}} \cap \widetilde{E_{2}} \leq \widetilde{K} .
$$

Since $\left(E_{1} \cap E_{2}\right) / Z(K)$ has order 4 , we have a contradiction to (7.2.1) in this case as well. Hence $K / Z(K) \neq \operatorname{Mat}(22)$ or $\operatorname{Mat}(23)$.

Assume that $K / Z(K) \cong \operatorname{Mat}(12)$. In this case

$$
L_{K} / Z(K) \sim 2_{+}^{1+4} \cdot \operatorname{Sym}(3)
$$

and by [6. Table $5.3 \mathrm{~b}$, notes 2$]$ an element $\tau$ of order 3 in $L_{K}$ acts fixed point freely on $O_{2}\left(L_{K} / Z(K)\right) / Z\left(O_{2}\left(L_{K} / Z(K)\right)\right)$.

Set $U_{1}=\left\langle Y_{M}^{L_{K}}\right\rangle \leq U_{Q}$. Then $U_{1}$ is elementary abelian. If some involution $u$ of $U_{1}$ induces an outer automorphism of $K$, then so does some involution of $C_{U_{1}}(\tau)$; however, $\tau$ is in the $K$-conjugacy class $3 A$ whereas the elements of order 3 in $C_{K}(u)$ are in the class $3 B$ (see [6, Table $5.3 \mathrm{~b}$, notes 3]). Therefore $\widetilde{U_{1}} \leq \widetilde{K}$. The action of $\tau$ now shows that $\left|\widetilde{U_{1}}\right|=8$. Hence by (7.2.1)

$$
\widetilde{Y_{M}}=\widetilde{U_{1}}
$$

We have

$$
\widetilde{O_{2}(M)} \leq C_{K \widetilde{O_{2}(M)}}\left(\widetilde{U_{1}}\right)
$$

which has order at most $2^{4}$, as $m_{2}(\operatorname{Aut}(\operatorname{Mat}(12))) \leq 4$ by [6. Table 5.6.1]. But then $\widetilde{O_{2}(M)^{\prime}}=1$ whereas we know it contains $\widetilde{z}$, a contradiction. Hence $K / Z(K) \varsubsetneqq \operatorname{Mat}(12)$. 


\section{Groups of Lie type in odd Characteristic as COMPONENTS}

The aim of this section is to show that if $K / Z(K)$ is a group of Lie type defined in odd characteristic, then $K \cong{ }^{2} \mathrm{G}_{2}(3)^{\prime} \cong \mathrm{SL}_{2}(8)$.

Lemma 8.1. The following statements hold.

(i) $K / Z(K) \nRightarrow \mathrm{PSL}_{2}(p)$ with $p \geq 7$ a Fermat or Mersenne prime.

(ii) If $K / Z(K) \cong \operatorname{PSL}_{2}(9)$, then $|Z(K)|$ is odd and

$$
K \widehat{N_{C_{S}(y)}}(K) \cong \operatorname{Sym}(6) \text { or } \operatorname{Aut}\left(\operatorname{PSL}_{2}(9)\right) \text {. }
$$

(iii) If $K / Z(K) \cong \mathrm{PSL}_{2}(5)$, then $Z(K)=1, Y_{M} \leq K C_{C_{S}(y)}(K)$ and $\widetilde{Y_{M}}=\widetilde{S \cap K}$.

Proof. Suppose that $K$ is one of the groups itemised in the lemma with $K N_{C_{S}(y)}(K) \nsucceq \operatorname{Sym}(6)$ or $\operatorname{Aut}\left(\operatorname{PSL}_{2}(9)\right)$. Thus, if $K \cong \operatorname{PSL}_{2}(p)$, then $K \widehat{N_{C_{S}(y)}}(K) \cong \operatorname{PSL}_{2}(p)$ or $\mathrm{PGL}_{2}(p)$ and, if $K \cong \operatorname{PSL}_{2}(9)$, we have

$$
K \widetilde{N_{C_{S}(y)}}(K) \cong X \in\left\{\operatorname{Alt}(6), \operatorname{PGL}_{2}(9), \operatorname{Mat}(10)\right\} \text {. }
$$

Assume that $z$ induces an outer automorphism on $K$. Then, as Mat(10) has semidihedral Sylow 2-subgroups, we have $K\langle z\rangle / Z(K) \cong \mathrm{PGL}_{2}(p)$ or $\mathrm{PGL}_{2}(9)$ and, in particular, the Sylow 2-subgroups of $K\langle z\rangle / Z(K)$ are dihedral groups of order at least 8 . Since $z Z(K)$ centralizes $(S \cap$ $K) Z(K) / Z(K)$, this is impossible. Hence $z$ induces an inner automorphism on $K$. In particular, Lemma 5.1 yields $Z(K)$ is a 2-group.

Assume that $Z(K) \neq 1$. Then $S \cap K$ is a quaternion group. Since $K=$ $[K, z]$, we have $z=w s$ for some $w \in C_{\langle z\rangle K}(K)$ and $s \in(S \cap K) \backslash Z(K)$. As $[S \cap K, z]=1$, we have $[S \cap K, s]=1$, a contradiction. Hence

$$
Z(K)=1 \text {. }
$$

We first prove parts (i) and (ii). By Lemma 5.16 as $S \cap K$ is not elementary abelian, $Y_{M}$ normalizes all the components of $E_{y}$ and by Lemma 5.25 we have

$$
\left|\widetilde{Y_{M}}\right|>2
$$

If $\widetilde{K} \nsucceq \mathrm{PSL}_{2}(7)$ or $\operatorname{PSL}_{2}(9)$, then, as $\widetilde{Y_{M}}$ is normalized by $\widetilde{N_{C_{S}(y)}(K)}$ the structure of the Sylow 2-subgroup of $\widetilde{K}$ shows that the only normal elementary abelian 2-subgroup has order 2 and so $\left|\widetilde{Y_{M}}\right| \leq 2$, which is not the case.

Hence $\widetilde{K} \cong \operatorname{PSL}_{2}(7)$ or $\operatorname{PSL}_{2}(9), \widetilde{S \cap K} \cong \operatorname{Dih}(8)$ and $\left|\widetilde{Y_{M}}\right|=4$. Thus $\left[S \cap K, Y_{M}\right]=Z(S \cap K)$ and so $O_{2}(M)$ normalizes $K$. Since $\widetilde{O_{2}(M)}$ centralizes $\widetilde{Y_{M}}, \widetilde{O_{2}(M)}=\widetilde{Y_{M}}$. But as $z \in O_{2}(M)^{\prime}$, we get $z \in C_{S}(K)$ 
which is a contradiction to Lemma 4.7. Thus (i) holds and to complete the proof of (ii) we just have to establish that, if $\widetilde{K_{C_{S}(y)}(K)} \cong \operatorname{Sym}(6)$ or Aut $\left(\mathrm{PSL}_{2}(9)\right)$, then $|Z(K)|$ is odd. Since $z$ centralizes $S \cap K$, we have that $K\langle z\rangle / C_{K\langle z\rangle}(K) \cong \mathrm{PSL}_{2}(9)$ or $\operatorname{Sym}(6)$. That $|Z(K)|$ is odd follows from these observations and [6, Proposition 5.2.8 (b)].

For the proof of (iii), we have already shown that $Z(K)=1$ and so $K \cong \operatorname{PSL}_{2}(5)$. Thus $N_{C_{G}(y)}(S \cap K) \cong \operatorname{Alt}(4)$ or $\operatorname{Sym}(4)$. Lemmas 5.12 and 5.14 imply that $Y_{M}$ normalizes $K$ and $Y_{M} \leq O_{2}\left(N_{G}\left(S_{y} T_{y}\right)\right)$. Hence (iii) holds. It follows from Lemma $\overline{5.25}$ that $\widetilde{Y_{M}}=\widetilde{S \cap K}$.

Lemma 8.2. We have $K / Z(K) \neq \mathrm{PSL}_{2}(5)$.

Proof. Assume $K / Z(K) \cong \mathrm{PSL}_{2}(5)$. By Lemma 8.1 we have that $K \cong$ $\mathrm{PSL}_{2}(5)$. Furthermore $\widetilde{Y_{M}}=\widetilde{S \cap K}$ and this is true for all components $K$ of $E_{y}$. In particular, $\left[S_{y}, Y_{M}\right] \leq C_{K}\left(E_{y}\right) \cap E_{y}=1$ and so

$$
S_{y} \leq C_{S}\left(Y_{M}\right)=O_{2}(M) \text {. }
$$

Set $F_{y}=E_{y} O_{2}(M)$. Then $O_{2}(M)$ is a Sylow 2-subgroup of $F_{y}$. As $O\left(F_{y}\right)=1$ we have by Proposition 2.7 that $J\left(O_{2}(M)\right)$ normalizes every component of $E_{y}$. Since $J\left(O_{2}(M)\right)$ centralizes $Y_{M}$, for any fixed component $K$ we have

$$
\left[\widetilde{S \cap K}, J\left(\widetilde{O_{2}(M)}\right)\right]=\left[\widetilde{Y_{M}}, J\left(\widetilde{O_{2}(M)}\right)\right]=1
$$

and so $J\left(\widetilde{O_{2}(M)}\right)=\widetilde{Y_{M}}$. Therefore $\Phi\left(J\left(O_{2}(M)\right)\right) \leq C_{C_{S}(y)}(K)$. Lemma 4.7 implies $J\left(\mathrm{O}_{2}(\mathrm{M})\right)$ is elementary abelian. Therefore

$$
J\left(O_{2}(M)\right)=S_{y} \times J\left(O_{2}(M) \cap T_{y}\right)
$$

and so $N_{E_{y}}\left(S_{y}\right) \leq N_{G}\left(J\left(O_{2}(M)\right)\right) \leq M^{\dagger}$. Now the action of $N_{E_{y}}\left(S_{y}\right)$ on $S_{y}$ yields $Y_{M} \cap E_{y}=S_{y}$ and $O_{2}(M)=C_{O_{2}(M)}(K) \times S_{y}$. In particular, $\widetilde{O_{2}(M)}$ is abelian. Then $z \in O_{2}(M)^{\prime}$ is contained in $C_{S}(K)$, which contradicts Lemma 4.7. Hence $K / Z(K) \nRightarrow \mathrm{PSL}_{2}(5)$.

Lemma 8.3. We cannot have $K / Z(K) \cong \mathrm{PSL}_{2}(9)$.

Proof. Assume $K / Z(K) \cong \mathrm{PSL}_{2}(9)$. By Lemma 5.16, $K$ is normalized

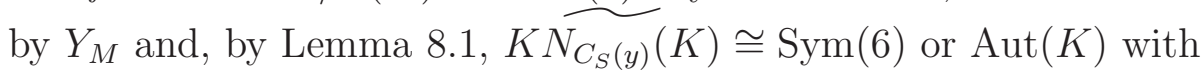

$$
|Z(K)| \text { is odd. }
$$

Furthermore, by Lemma 5.25 we have $\left|\widetilde{Y_{M}}\right| \geq 4$.

Assume that $\left[\widetilde{S \cap K}, \widetilde{Y_{M}}\right] \neq 1$. Then $K \geq\left[S \cap K, Y_{M}\right] \neq 1$ and so $O_{2}(M)$ normalizes $K$ by Lemma 5.18. Since $z \in O_{2}(M)^{\prime}$ and $\widetilde{O_{2}(M)^{\prime}} \leq$ $\widetilde{K}$, we have $\widetilde{z} \in \widetilde{K}$. Now Lemma $\left[5.25\right.$ implies that $\widetilde{O_{2}(M)^{\prime}} \cap \widetilde{Y_{M}}$ has 
order 4. But then, as $\widetilde{O_{2}(M)}$ centralizes $\widetilde{Y_{M}}$, we have $\widetilde{O_{2}(M)}$ is abelian. As $z \in O_{2}(M)^{\prime}$, we then get that $z \in C_{S}(K)$, contradicting Lemma 4.7. Hence

$$
\left[\widetilde{S \cap K}, \widetilde{Y_{M}}\right]=1 \text {. }
$$

As $\left|\widetilde{Y_{M}}\right| \geq 4$ by Lemma $\left[5.25\right.$ and $\left[\widetilde{S \cap K}, \widetilde{Y_{M}}\right]=1$, we have $\left|\widetilde{Y_{M}}\right|=4$ and $\widetilde{Y_{M}}$ maps to the centre of a Sylow 2-subgroup of Sym(6). In particular, $S \cap K Y_{M}$ is contained in $O_{2}(M)$. This applies to every component of $E_{y}$. Especially

$$
S_{y} \leq O_{2}(M) .
$$

If $z$ does not induce an inner automorphism on $K$, then $O^{2}\left(L_{K}\right) \cong$ Alt(4). By Lemma 5.21 we have that $O_{2}(M)$ normalizes $K$, which contradicts $z \in O_{2}(M)^{\prime}$. Thus $z$ induces an inner automorphism and so by Lemma $5.10(K)=1$. Now by [5, Remark following Proposition 8.5] the assumptions of Proposition 2.7 are satisfied, which yields that $J\left(O_{2}(M)\right)$ normalizes every component of $E_{y}$. Hence $J\left(\widetilde{O_{2}(M)}\right) \leq$ $J\left(\widehat{N_{C_{S}(y)}(K)}\right) \cong \operatorname{Dih}(8) \times 2$. Thus

$$
\left.\mid \Phi\left(J \widetilde{\left(O_{2}(M)\right.}\right)\right) \mid \leq 2 .
$$

Let $A$ be a maximal elementary abelian subgroup of $O_{2}(M)$. Then $A$ normalizes $K$ and

$$
m_{2}(A)=m_{2}\left(C_{A K}(K)\right)+m_{2}\left(A K / C_{A K}(K)\right)
$$

. Combining this with (1) we conclude that $J(A K)=A(S \cap K)$. In particular, $J\left(O_{2}(M)\right)$ is not abelian.

As $\Phi\left(J\left(O_{2}(M)\right)\right) \neq 1$, we may select $z_{*} \in C_{Y_{M} \cap \Phi\left(J\left(O_{2}(M)\right)\right)}(S)^{\#}$, and obtain

$$
\widetilde{\left\langle z_{*}{ }^{M}\right\rangle} \leq \Phi\left(J\left(\widetilde{O_{2}(M)}\right)\right)
$$

contrary to (21) and Lemma 5.25. Hence $K / Z(K) \neq \mathrm{PSL}_{2}(9)$.

Lemma 8.4. We cannot have $K / Z(K) \cong \operatorname{PSL}_{2}\left(p^{a}\right)$ with $p$ an odd prime.

Proof. Suppose that $K / Z(K) \cong \mathrm{PSL}_{2}\left(p^{a}\right)$. By Lemmas 8.1, 8.2 and 8.3. $p^{a}$ is not a Mersenne or Fermat prime and $p^{a} \neq 9$. If $z$ induces an inner automorphism on $K$, then $L_{K}$ has a normal 2-complement. Application of Lemma 4.7 (v) yields that $L_{K}$ is a 2-group. Now [8, Hauptsatz 8.27] implies that $p^{a}$ is a Fermat or Mersenne prime or $p^{a}=$ 9 , a contradiction. 
Hence $z$ induces an outer automorphism on $K$. If $z$ induces an innerdiagonal automorphism, then $\langle z\rangle K / Z(K)$ has non-abelian dihedral Sylow 2-subgroups. Since $z$ induces an outer automorphism which centralizes $S \cap K$ this is impossible.

Hence $z$ is in the coset of the field automorphism $\left(\bmod \operatorname{PGL}_{2}\left(p^{a}\right)\right)$ and hence is a field automorphism by [6, Proposition 4.9.1]. Thus, as $p^{a} \neq 9, F^{*}\left(L_{K} Z(K) / Z(K)\right) \cong \mathrm{PSL}_{2}\left(p^{a / 2}\right)$ and this contradicts Lemma 4.7(i). Hence $K / Z(K) \nRightarrow \mathrm{PSL}_{2}\left(p^{a}\right)$.

Proposition 8.5. If $K / Z(K)$ is a group of Lie type in odd characteristic, then $K / Z(K) \cong{ }^{2} \mathrm{G}_{2}(3)^{\prime} \cong \mathrm{PSL}_{2}(8)$.

Proof. By Lemma 8.4 we may assume that $K / Z(K) \nsucceq \operatorname{PSL}_{2}\left(p^{a}\right)$ and we also suppose that $K / Z(K) \neq{ }^{2} \mathrm{G}_{2}(3)^{\prime}$. We know that $F^{*}\left(L_{K}\right)$ is a 2 -group by Lemma $4.7(\mathrm{v})$. Using Lemma 3.20 yields $K / Z(K)$ is one of the following groups.

$\mathrm{PSL}_{3}(3), \mathrm{PSU}_{3}(3), \mathrm{PSL}_{4}(3), \mathrm{PSU}_{4}(3), \mathrm{PSp}_{4}(3), \mathrm{P}_{7}(3), \mathrm{P}_{8}^{+}(3), \mathrm{G}_{2}(3)$.

Furthermore, in each case the conjugacy class of $\widetilde{z}$ is uniquely determined and is contained in $\widetilde{K}$. Using [6, Table 4.5.1] with Lemma 3.20 we have

$$
\widetilde{O_{2}\left(L_{K}\right)}= \begin{cases}\mathrm{Q}_{8} & K / Z(K) \cong \mathrm{PSL}_{3}(3) \\ \mathrm{Q}_{8} \circ 4 & K / Z(K) \cong \mathrm{PSU}_{3}(3) \\ 2_{+}^{1+4} & K / Z(K) \cong \mathrm{PSL}_{4}(3), \mathrm{PSU}_{4}(3), \mathrm{PSp}_{4}(3), \mathrm{G}_{2}(3) \\ 2_{+}^{1+4} \times 2^{2} & K / Z(K) \cong \mathrm{P}_{7}(3) \\ 2_{+}^{1+8} & K / Z(K) \cong \mathrm{P}_{8}^{+}(3)\end{cases}
$$

Moreover, other than for $K / Z(K) \cong \mathrm{P} \Omega_{7}(3), \widetilde{L_{K}}$ does not normalize any elementary abelian subgroup of $\widetilde{O_{2}\left(L_{K}\right)}$ of order greater that 2 .

Suppose that $K \neq \mathrm{P} \Omega_{7}(3)$. Then

$$
\left.\widetilde{U}_{Q} \cap \widetilde{O_{2}\left(L_{K}\right.}\right)=\Omega_{1}\left(Z\left(\widetilde{O_{2}\left(L_{K}\right)}\right)\right)=\Omega_{1}\left(Z\left(\widetilde{L_{K}}\right)\right)
$$

which has order 2. Hence $O^{2}\left(L_{K}\right)$ centralizes $U_{Q}$ and so also $Y_{M}$. Applying Lemma 5.22 (iv) provides a contradiction.

Therefore $\widetilde{K} \cong \mathrm{P} \Omega_{7}(3)$. Then $O^{2}\left(\widetilde{L_{K}}\right) \cong \operatorname{Alt}(4) \times\left(\mathrm{SL}_{2}(3) \circ \mathrm{SL}_{2}(3)\right)$. Set $J=O^{2}\left(C_{O^{2}\left(\widetilde{L_{K}}\right)}\left(Z\left(O_{2}\left(O^{2}\left(\widetilde{L_{K}}\right)\right)\right)\right)\right.$. Then $J \cong \mathrm{SL}_{2}(3) \circ \mathrm{SL}_{2}(3)$ and $O^{2}(J)=J$ centralizes every abelian subgroup of $O_{2}\left(O^{2}\left(\widetilde{L_{K}}\right)\right)$ which it normalizes. In particular, $J$ centralizes $U_{Q} \geq Y_{M}$. Thus Lemma 5.22 (iv) provides a contradiction. This completes the proof of the proposition.

The group ${ }^{2} \mathrm{G}_{2}(3)^{\prime}$ will be handled as $\mathrm{PSL}_{2}(8)$ in Section 10 . 


\section{Alternating groups as Components}

In this section we will show that $K / Z(K)$ is not an alternating group $\operatorname{Alt}(n), n \geq 5$. The cases $n=5,6$ have been discussed in Lemma 8.2 and Lemma 8.3. Thus we may assume that $n \geq 7$. Therefore $K \widehat{N_{C_{S}(y)}}(K)$ is isomorphic to either $\operatorname{Alt}(n)$ or $\operatorname{Sym}(n)$.

Lemma 9.1. We have $C_{G}\left(Y_{M}\right) C_{Q}(y)$ normalizes $K$.

Proof. We consider $X \cong \operatorname{Sym}(n)$. Then, as $n \geq 7$, every involution in $X$ either centralizes an element of cycle shape 3 or $3^{2}$. Hence $L_{K}$ is not a 2-group. Lemma 5.21 gives the result.

Because $O_{2}(M)$ normalizes $K$ by Lemma 9.1 and $\widetilde{z} \in O_{2}(M)^{\prime}, \widetilde{K\langle z\rangle}=$ $\widetilde{K}$ is isomorphic to $\operatorname{Alt}(n)$. Under this isomorphism, we get $\widetilde{z}$ is even and we let $\operatorname{supp}(z)$ be the set of elements of $\{1, \ldots, n\}$ moved by the image of $z$. For a subgroup $H$ of $\operatorname{Sym}(n)$, we use $H^{e}$ to denote the subgroup of even elements of $H$. We set notation so that $|\operatorname{supp}(z)|=2 \mathrm{~m}$.

Lemma 9.2. We have $n>7$ and $Z(K)=1$.

Proof. If $n=7$, then as $\widetilde{z}$ is even, we get $m=2$ and then $O_{3}\left(C_{K}(z)\right) \neq 1$, which contradicts Lemma 4.7. Thus $n>7$.

We have $K=[K, z]$ by Lemma 5.1 and so $z$ induces a non-trivial automorphism of $K$ of order 2 and $z$ centralizes $S \cap K \in \operatorname{Syl}_{2}(K)$. Application of [6, Proposition 5.2.8 (b)] implies that $Z(K)=1$.

Lemma 9.3. We have $n-2 m \leq 2$ and, if $2 m=n-2$, then $n \equiv$ $2(\bmod 4)$. Furthermore either $\mathrm{O}_{2}\left(L_{K}\right) / Z(K)$ is elementary abelian and involves exactly one non-trivial irreducible $O^{2}\left(L_{K}\right)$-module or $n \in$ $\{8,9,10\}$ and $|\operatorname{supp}(z)|=8$.

Proof. By Lemma $9.2 Z(K)=1$, so $K \cong \operatorname{Alt}(n)$. If $2 m \leq n-4$, then $O^{2}\left(F^{*}\left(L_{K}\right)\right)$ contains Alt $(n-2 m)$ and this contradicts Lemma $4.7(\mathrm{v})$, other than if $2 m=n-4$.

Suppose that $2 m=n-4$. We may assume that $z=(12)(34) \ldots(n-$ $5, n-4)$. Then $L_{K} \cong(2 \imath \operatorname{Sym}(m) \times \operatorname{Sym}(4))^{e}$, which contains a Sylow 2 -subgroup of $K$ only if $n \equiv 4(\bmod 8)$. By Lemma 9.1 we have that $\mathrm{O}_{2}(M)$ normalizes $K$. We consider $H, J \in \mathcal{L}_{K}(S \cap K)$ with $H$ stabilizing the partition $\{\{1,2\}, \ldots\{n-1, n\}\}$ and $J$ stabilizing $\{\{1,2,3,4\}, \ldots,\{n-$ $3, n-2, n-1, n\}$. Then $H$ and $J$ are normalized by $O_{2}(M)$. By Lemma 4.8. $Y_{M} \leq \mathrm{O}_{2}\left(\mathrm{HO}_{2}(M)\right) \cap \mathrm{O}_{2}\left(J O_{2}(M)\right)$. This shows that $\widetilde{Y_{M}}$ is contained in the subgroup

$$
\langle(12)(34), \ldots,(n-3, n-2)(n-1, n)\rangle .
$$


In particular any subgroup of $Y_{M}$, which is normalized by $O^{2}\left(L_{K}\right)$ is centralized by $O^{2}\left(L_{K}\right)$. By Lemma 5.20, $Q$ normalizes $O^{2}\left(L_{K}\right)$. But then it also normalizes the fours-group

$$
\langle(n-3, n-2)(n-1, n),(n-3, n-1)(n-2, n)\rangle,
$$

as this subgroup is obviously characteristic in $O^{2}\left(L_{K}\right)$. This is trivial to observe if $2 m>8$. In the case $2 m=8$, it is $\left[Z\left(O_{2}\left(O^{2}\left(L_{K}\right)\right)\right), O^{2}\left(L_{K}\right)\right]$ which is also characteristic. Therefore there exists $z_{1} \in Z(Q)^{\#}$ with $\left|\operatorname{supp}\left(z_{1}\right)\right|=4$, a contradiction to Lemma 4.7(v).

Therefore $|\operatorname{supp}(z)| \geq n-3$. If $|\operatorname{supp}(z)|=n-3$, then $O\left(L_{K}\right) \neq 1$, and we have a contradiction to Lemma 4.7 . Hence $|\operatorname{supp}(z)| \geq n-$ 2. If $2 m \neq n-2$, we have that $C_{K}(z) \cong(2 \succ \operatorname{Sym}(m))^{e}$. If $2 m=$ $n-2$, then $C_{K}(z) \cong(2 \times 2 \imath \operatorname{Sym}(m))^{e}$ which contains a Sylow 2subgroup of $K$ only if $n \equiv 2(\bmod 4)$. As $n \geq 7$, we have $m \geq 3$. Now $\operatorname{Sym}(m)$ has a non-trivial normal 2-subgroup if and only if $m=4$. Thus so long as $m \neq 4$, we have that $O_{2}\left(L_{K}\right)$ is elementary abelian and $L_{K} / O_{2}\left(L_{K}\right) \cong \operatorname{Sym}(m)$ induces the non-trivial irreducible part of the natural permutation module on the unique non-central chief factor in $O_{2}\left(L_{K}\right)$. Finally we note that we have $m=4$ only when $n \in\{8,9,10\}$.

We now deal with the three exceptional cases in Lemma 9.3 ,

Lemma 9.4. We have $|\operatorname{supp}(z)| \neq 8$. In particular $n>10$.

Proof. Suppose that $|\operatorname{supp}(z)|=8$. Then by Lemma 9.3, $n \in\{8,9,10\}$. By Lemma $9.2 Z(K)=1$. We may suppose that $z$ corresponds to the permutation (12)(34)(56)(78). By Lemma 9.1, $O_{2}(M)$ normalizes $K$.

To start assume that $\widetilde{K} \cong \operatorname{Alt}(8)$ or $\operatorname{Alt}(9)$. Then there exist $J_{1}, J_{2} \in$ $\mathcal{L}_{K}(S \cap K)$ with $J_{1} \cong J_{2} \cong 2^{3}: \mathrm{SL}_{3}(2)$ and $J_{1} \neq J_{2}$. Both these subgroups are normalized by $\mathrm{O}_{2}(M)$ and hence

$$
Y_{M} \leq O_{2}\left(Y_{M} J_{1}\right) \cap O_{2}\left(Y_{M} J_{2}\right)=C_{Y_{M} K}(K)\left(O_{2}\left(J_{1}\right) \cap O_{2}\left(J_{2}\right)\right)
$$

by Lemma 4.8. Since $\left|O_{2}\left(J_{1}\right) \cap O_{2}\left(J_{2}\right)\right|=2$, this contradicts Lemma 5.25. Hence

$$
\widetilde{K O_{2}(M)} \cong \operatorname{Sym}(8), \operatorname{Sym}(9), \operatorname{Alt}(10), \text { or } \operatorname{Sym}(10) .
$$

Notice that in $\operatorname{Alt}(10),(\operatorname{Sym}(8) \times \operatorname{Sym}(2))^{e} \cong \operatorname{Sym}(8)$.

We consider $J \in \mathcal{L}_{K}(S \cap K)$ stabilizing the partition

$$
\left\{\{1,2,3,4\},\{5,6,7,8\}, \Omega_{0}\right\}
$$

where $\left|\Omega_{0}\right| \in\{0,1,2\}$. Then

$$
J \cong\left\{\begin{array}{ll}
(\operatorname{Sym}(4) \prec 2)^{e} & n \in\{8,9\} \\
(\operatorname{Sym}(4) \prec 2 \times 2)^{e} & n=10
\end{array} .\right.
$$


Notice that $J$ has characteristic 2 and is normalized by $O_{2}(M)$. Setting $J_{1}=O_{2}\left(J O_{2}(M)\right)$, we have $Y_{M} \leq J_{1}$ and $\left\langle Y_{M}^{J}\right\rangle$ is elementary abelian by Lemma 4.8. We calculate

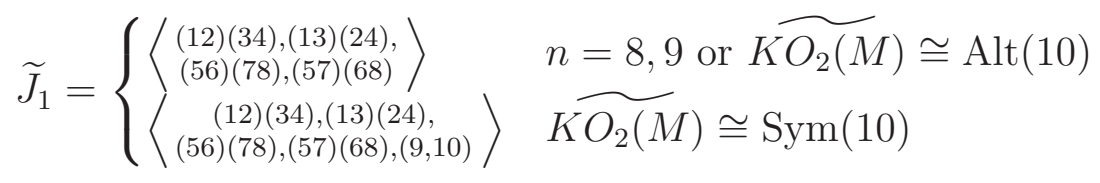

and

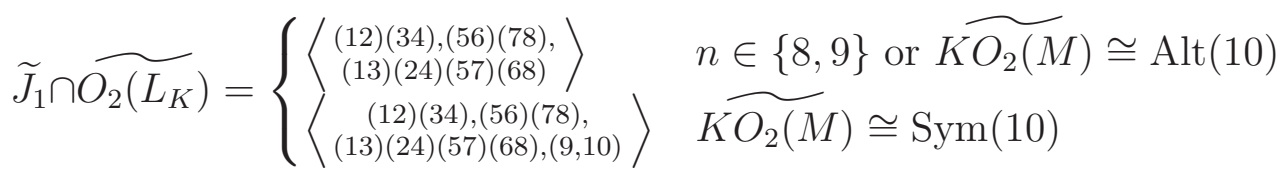

which has order 8 in the first cases and 16 in the second. As $L_{K} \leq$ $N_{G}(Q)$, the projection $\widetilde{Y_{M}}$ is contained in $\widetilde{J_{1}} \cap \widetilde{O_{2}\left(L_{K}\right)}$.

Suppose that $n \in\{8,9\}$ or $\widetilde{K O_{2}(M)} \cong \operatorname{Alt}(10)$. Then we have $\left|C_{\widetilde{K}}\left(O^{2}\left(\widetilde{L_{K}}\right)\right)\right|=2$ and so, as $\widetilde{Y_{M}} \leq \widetilde{K}$, Lemma 5.26 (ii) applies to give

$$
\widetilde{Y_{M}}=\widetilde{J_{1}} \cap \widetilde{O_{2}\left(L_{K}\right)} .
$$

Pick $\rho \in L_{K}$ corresponding to $(1,3,5)(2,4,6)$. As $(13)(24)(57)(68) \in$ $\widetilde{Y_{M}}$

$$
(3,5)(4,6)(1,7)(2,8)=((13)(24)(57)(68))^{\rho} \in \widetilde{\left\langle Y_{M}^{L_{K}}\right\rangle} .
$$

Since $(12)(34)$ and $(3,5)(4,6)(1,7)(2,8)$ do not commute, we have a contradiction to $\widetilde{\left\langle Y_{M}^{L_{K}}\right\rangle} \leq \widetilde{U_{Q}}$ being abelian. Hence $\widetilde{K O_{2}(M)} \cong \operatorname{Sym}(10)$.

Let $\widetilde{H} \leq \widehat{\mathrm{KO}_{2}(M)}$ be the subgroup that preserves the partition

$$
\{\{1,2\},\{3,4\},\{5,6\},\{7,8\},\{9,10\}\} .
$$

Then $\widetilde{H} \cong 2$ \Sym(5), and $\widetilde{Y_{M}} \leq \widetilde{O_{2}(H)}$ and we have

$$
\widetilde{Y_{M}} \leq \widetilde{J_{1}} \cap \widetilde{O_{2}(H)} \leq\langle(12)(34),(56)(78),(9,10)\rangle
$$

which has order 8 . Notice that $\widetilde{z}$ is the only $\widehat{\mathrm{KO}_{2}(M)}$-conjugate of $\widetilde{z}$ in $\widetilde{Y_{M}}$.

By the choice of $z$ we have $z \in Y_{M} \cap O_{2}(M)^{\prime}$. By Lemma 5.25, there exists $m \in M$ such that $\widetilde{z^{m}} \neq \widetilde{z}$. Obviously $z^{m} \in Y_{M} \cap O_{2}(M)^{\prime}$ and so

$$
\widetilde{z} \in O_{2}\left(\widetilde{M)^{\prime} \cap} Y_{M} \leq \widetilde{K} \cong \operatorname{Alt}(10)\right. \text {. }
$$

Hence $\widetilde{z^{m}}$ corresponds to an element of cycle type $2^{2}$. However this means $C_{K}\left(z^{m}\right)$ contains a component isomorphic to Alt(6) and this contradicts Lemma $4.7(\mathrm{v})$. 
Assume now $n=10$. Then $|\operatorname{supp}(z)| \neq 8$. By Lemma 9.3 this gives $|\operatorname{supp}(z)|=10$, which contradicts $\widetilde{z} \in \widetilde{K}$.

Proposition 9.5. We have $K / Z(K)$ is not an alternating group.

Proof. By Lemma 9.4 we have $n>10$. Further Lemma 9.2 gives us $Z(K)=1$. By the choice of $z$ we have $\widetilde{K\langle z\rangle}=\widetilde{K}$.

Assume that $\widetilde{Y_{M}}$ covers the unique non-trivial irreducible $O^{2}\left(L_{K}\right)$ module in $\widetilde{O_{2}\left(L_{K}\right)}$. Then $C_{\widetilde{K}}\left(\widetilde{Y_{M}}\right)=O_{2}\left(\widetilde{L_{K}}\right)$. By Lemma 9.1 we have that $O_{2}(M)$ normalizes $K$ and so $\widetilde{O_{2}(M)} \leq C_{\widetilde{K}}\left(\widetilde{Y_{M}}\right)$ is elementary abelian. Therefore $z \in O_{2}(M)^{\prime} \leq C_{S}(K)$, which is impossible.

Hence $\widetilde{Y_{M}}$ does not cover the non-trivial irreducible $O^{2}\left(L_{K}\right)$-module in $\widetilde{O_{2}\left(L_{K}\right)}$ and so any $O^{2}\left(L_{K}\right)$-invariant subgroup $W$ of $Y_{M}$ is centralized by $O^{2}\left(L_{K}\right)$. Therefore Lemma 5.20 yields $O^{2}\left(L_{K}\right)$ is normalized by $Q$. Since $\mathrm{O}_{2}\left(\mathrm{O}^{2}\left(L_{K}\right)\right)$ is elementary abelian and contains exactly one non-central $O^{2}\left(L_{K}\right)$-chief factor, Lemma 5.24 yields

$$
\left[O_{2}\left(O^{2}\left(L_{K}\right)\right), O^{2}\left(L_{K}\right)\right] \leq Z(Q) .
$$

We now notice

$$
\left[O_{2}\left(O^{2}\left(L_{K}\right)\right), O^{2}\left(L_{K}\right)\right]=O_{2}\left(O^{2}\left(L_{K}\right)\right)
$$

contains an element $w$ which is $K$-conjugate to the permutation $(12)(34)$. As $w \in Z(Q), C_{G}(w)$ has characteristic 2 , hence $C_{C_{G}(y)}(w)$ has characteristic 2 by Lemma 4.7 (vi) which it plainly does not. This contradiction shows that $K / Z(K)$ is not an alternating group.

\section{Groups of Lie type in CharaCteristic 2 AS COMPONEnts}

In this section we tackle the possibility that $K / Z(K)$ is a group of Lie type in characteristic two. Some of these groups have been considered before under different names. For example $\mathrm{L}_{2}(4) \cong \mathrm{L}_{2}(5) \cong \operatorname{Alt}(5)$, $\mathrm{PSp}_{4}(2)^{\prime} \cong \operatorname{Alt}(6), \mathrm{L}_{3}(2) \cong \mathrm{L}_{2}(7), \mathrm{G}_{2}(2)^{\prime} \cong \mathrm{U}_{3}(3), \mathrm{L}_{4}(2) \cong \Omega_{6}^{+}(2) \cong$ $\operatorname{Alt}(8)$ and $\Omega_{6}^{-}(2) \cong \mathrm{U}_{4}(2) \cong \operatorname{PSp}_{4}(3)$.

We will start with the groups $\mathrm{SL}_{2}\left(2^{a}\right)$ and ${ }^{2} \mathrm{~B}_{2}\left(2^{a}\right)$, which then also handles the case of ${ }^{2} \mathrm{G}_{2}(3)^{\prime}$ which was left open in Proposition 8.5.

Lemma 10.1. Suppose that $K / Z(K) \cong \mathrm{SL}_{2}\left(2^{a}\right)$ or ${ }^{2} \mathrm{~B}_{2}\left(2^{a}\right), a \geq 3$. Then

(i) $Y_{M} \leq \Omega_{1}\left(S_{y}\right) T_{y}$;

(ii) $K$ is simple; and

(iii) $S_{y} \leq O_{2}(M)$. 
Proof. By Lemmas 3.11 and 5.14, $N_{G}\left(S_{y} T_{y}\right)$ has characteristic 2. Lemma 5.12 yields $Y_{M} \leq O_{2}\left(N_{G}\left(S_{y} T_{y}\right)\right)$ and $Y_{M}$ normalizes every component of $E_{y}$. In particular, $Y_{M}$ induces inner automorphisms on each of such component. It follows that $Y_{M} \leq \Omega_{1}\left(S_{y}\right) T_{y}$. This proves (i).

Assume that $Z(K) \neq 1$. By [6. Table 6.1.3], $K / Z(K) \cong{ }^{2} \mathrm{~B}_{2}(8)$, as $a \geq 3$. Furthermore by Lemma $3.19 Z\left(S_{y} \cap K\right)=Z(K)$.

Hence $K\langle z\rangle=K C_{K\langle z\rangle}(K), z \notin K$ and $z \notin C_{K\langle z\rangle}(K)$. Thus $z=a b$ where $a \in S \cap K$ and $b \in C_{K\langle z\rangle}(K)$. As $z$ and $C_{K\langle z\rangle}(K)$ centralize $S \cap K$, so does $a$. Therefore $a \in Z(S \cap K)=Z(K)$ and we conclude $z \in C_{K\langle z\rangle}(K)$, a contradiction. This proves (ii).

By (ii) $K$ is simple. Since $S_{y}$ centralizes $\Omega_{1}\left(S_{y}\right) T_{y} \geq Y_{M}$, we have $S_{y} \leq S \cap C_{M}\left(Y_{M}\right)=O_{2}(M)$. This is (iii).

Lemma 10.2. We have that $K / Z(K) \neq \mathrm{SL}_{2}\left(2^{a}\right)$ or ${ }^{2} \mathrm{~B}_{2}\left(2^{a}\right)$ with $a \geq 3$.

Proof. Assume that $K / Z(K) \cong \mathrm{SL}_{2}\left(2^{a}\right)$ or ${ }^{2} \mathrm{~B}_{2}\left(2^{a}\right)$ with $a \geq 3$. By Lemma 10.1 (ii), $K$ is simple.

We first prove that $J\left(O_{2}(M)\right)=\Omega_{1}\left(S_{y}\right) \times J\left(T_{y}\right)$. By Lemma10.1(iii), $S_{y} \leq O_{2}(M)$ and so we consider $X=E_{y} O_{2}(M)$. We have $O_{2}(M) \in$ $\operatorname{Syl}_{2}(X)$. Using Lemmas 2.8 and 3.3, the fact that $a>2$ yields

$$
J\left(O_{2}(M)\right)=J\left(C_{O_{2}(M)}\left(E_{y}\right)\right) \times J\left(S_{y}\right) .
$$

Using Lemma 3.3 again gives $J\left(S_{y}\right)=\Omega_{1}\left(S_{y}\right)=Z\left(S_{y}\right)$. From the structure of $J\left(O_{2}(M)\right)$, we see that $N_{E_{y}}\left(S_{y}\right)$ normalizes $J\left(O_{2}(M)\right)$ and therefore $N_{E}\left(S_{y}\right) \leq M^{\dagger}$ by Lemma 4.3. Since $Y_{M}$ is normalized by $M^{\dagger}$, it is also normalized by $N_{E_{y}}\left(S_{y}\right)$. Therefore $\left[Y_{M}, N_{E_{y}}\left(S_{y}\right)\right] \leq Y_{M} \cap$ $E_{y}$. Since $Y_{M}$ does not centralize $N_{E_{y}}\left(S_{y}\right)$, we deduce that $\Omega_{1}\left(S_{y}\right)=$ $\left[Y_{M}, N_{E_{y}}\left(S_{y}\right)\right]<Y_{M}$. Now we have $J\left(O_{2}(M)\right)=J\left(C_{S}\left(E_{y}\right)\right) Y_{M}$. Thus

$$
\begin{aligned}
{\left[J\left(O_{2}(M)\right), O_{2}(M)\right] } & =\left[J\left(C_{O_{2}(M)}\left(E_{y}\right)\right) Y_{M}, O_{2}(M)\right] \\
& =\left[J\left(C_{O_{2}(M)}\left(E_{y}\right)\right), O_{2}(M)\right] \leq T_{y} .
\end{aligned}
$$

As $\left[J\left(O_{2}(M)\right), O_{2}(M)\right] \leq T_{y}$, Lemma 5.7 implies

$$
\left[J\left(O_{2}(M)\right), O_{2}(M)\right]=1 .
$$

Hence

$$
Y_{M} \leq J\left(O_{2}(M)\right) \leq \Omega_{1}\left(Z\left(O_{2}(M)\right)\right)=Y_{M} .
$$

But then $Y_{M}=J\left(O_{2}(M)\right)$ and this contradicts Lemma 4.11, The lemma is proved.

Lemma 10.3. We have $K / Z(K) \not \operatorname{PSU}_{3}(q)$ for $q=2^{a} \geq 4$.

Proof. Suppose that $K / Z(K) \cong \operatorname{PSU}_{3}(q)$ with $q \geq 4$. We have $Z(K)=$ 1 by [6, Table 6.1.3] and Lemma 5.1. We take facts about $K$ from [4, 5.4] and [8, II.10.12 Satz]. An important point is that $\Omega_{1}(S \cap K)=Z(S \cap K)$. 
We have $\left|L_{K}\right|=q^{3}(q+1) /(q+1,3)$ and so $L_{K}$ is not a 2-group. Therefore $C_{G}\left(Y_{M}\right) C_{Q}(y)$ normalizes $K$ by Lemma 5.21 .

We start with the following statement.

(10.3.1) Suppose that $A$ is an elementary abelian normal subgroup of $O_{2}(M) C_{Q}(y)$. Then $A \leq \Omega_{1}(S \cap K) C_{C_{S}(y)}(K)=Z(S \cap K) C_{C_{S}(y)}(K)$.

Since $A$ normalizes $K$, is elementary abelian, and no outer automorphism of $K$ centralizes $(S \cap K) / Z(S \cap K), A \leq(S \cap K) C_{C_{S}(y)}(K)$. Therefore

$$
A \leq \Omega_{1}(S \cap K) C_{C_{S}(y)}(K)=Z(S \cap K) C_{C_{S}(y)}(K) .
$$

By (10.3.1), $Y_{M} \leq Z(S \cap K) C_{C_{S}(y)}(K)$. As $Z(S \cap K)$ is centralized by $L_{K}$, we obtain

$$
O^{2}\left(L_{K}\right) \leq C_{G}\left(Y_{M}\right)
$$

Now, as $O_{2}(M)$ normalizes $L_{K}$, Lemma 5.22 (iii) yields $F^{*}\left(C_{G}(y)\right)=$ $K_{2}\left(C_{G}(y)\right)$ (and part (ii) leads to $q=8$, but we shall not use this).

Furthermore, (10.3.1) implies that $\left[S \cap K, Y_{M}\right] \leq S \cap K \cap C_{S}(K)=1$,

$$
S \cap K \leq C_{S}\left(Y_{M}\right)=O_{2}(M) .
$$

Assume $w \in O_{2}(M)$ has order 2 and induces an outer automorphism on $K$. Then, as $\mathrm{O}_{2}(M)$ normalizes $K$ and is contained in $S$, w acts on $N_{K}(S \cap K) /(S \cap K)$. By [6, Proposition 4.9.2 (b)(2) and (g)], $w$ is conjugate in $\operatorname{Aut}(K)$ to a standard graph automorphism and so $w$ centralizes $Z(S \cap K)$ (see [6, Theorem 2.5.1 d]) and $C_{K}(w) \cong \mathrm{SL}_{2}(q)$. Hence there is an element of $\nu \in N_{C_{K}(w)}(Z(S \cap K))$ of order $q-1$. Since the Sylow 2-subgroups of $K$ are trivial intersection subgroups in $K, \nu$ normalizes $S \cap K$. As $N_{K}(S \cap K) /(S \cap K)$ is cyclic, $\langle\nu\rangle(S \cap K)$ is uniquely determined by its order in $N_{K}(S \cap K) /(S \cap K)$, and so $\nu$ normalizes $\Omega_{1}\left(O_{2}(M)\right)(S \cap K)$. Therefore $\nu$ normalizes $\Omega_{1}\left(O_{2}(M)\right)$ as $S \cap K \leq O_{2}(M)$. It follows that $\nu \in N_{G}\left(\Omega_{1}\left(O_{2}(M)\right)\right) \leq M^{\dagger}$. Therefore $\nu$ normalizes $Y_{M^{\dagger}}=Y_{M}$. As $\langle\nu\rangle$ acts irreducibly on $Z(S \cap K)$ and normalizes $Y_{M}$, we have

$$
Y_{M} C_{C_{S}(y)}(K)=Z(S \cap K) C_{C_{S}(y)}(K) .
$$

Recall that $U_{Q}$ is elementary abelian. Hence $U_{Q} \leq O_{2}(M)$ and (10.3.1) implies that

$$
U_{Q} \leq Z(S \cap K) C_{C_{S}(y)}(K)=Y_{M} C_{C_{S}(y)}(K) \leq U_{Q} C_{C_{S}(y)}(K) .
$$

Therefore

$$
U_{Q} C_{C_{S}(y)}(K)=Y_{M} C_{C_{S}(y)}(K) .
$$


Hence $\left[U_{Q}, O_{2}(M)\right] \leq C_{C_{S}(y)}(K)$ and $\left[U_{Q}, O_{2}(M)\right]$ is normalized by $Q$. We conclude from Lemma 5.7 that $\left[U_{Q}, O_{2}(M)\right]=1$ and so $U_{Q}=Y_{M}$ and

$$
N_{G}(Q) \leq N_{G}\left(Y_{M}\right)=M^{\dagger} .
$$

Suppose that $J \in \mathcal{L}_{G}(S)$. Then, as $Y_{M}$ is not characteristic 2-tall, $Y_{M} \leq O_{2}(J)$. Hence $Y_{J} \leq C_{S}\left(Y_{M}\right)=O_{2}(M)$ and so $Y_{J} \leq Y_{M} C_{C_{S}(y)}(K)$ by (10.3.1), Thus $\left[O_{2}(M), Y_{J}\right] \leq C_{C_{S}(y)}(K)$ and again this is normalized by $Q$. Hence $\left[O_{2}(M), Y_{J}\right]=1$ and so $Y_{J} \leq Y_{M}$. This contradicts Lemma 4.10. Hence $K / Z(K) \neq \operatorname{PSU}_{3}(q)$.

Lemma 10.4. Suppose that $K / Z(K) \cong \operatorname{PSL}_{3}(q), q=2^{a} \geq 4$. Then $K / Z(K) \cong \mathrm{PSL}_{3}(4)$.

Proof. We assume that $q \geq 8$ and seek a contradiction. As $q \neq 4$, [6, Table 6.1.3] and Lemma 5.1 combine to give $Z(K)=1$. The structural information we require about $S \cap K$ is given in Lemma 3.10. From there we see that $S \cap K$ has exactly two elementary abelian subgroups of order $q^{2}$. Name these subgroups $E_{1}$ and $E_{2}$. Furthermore, every element of $(S \cap K) \backslash\left(E_{1} \cup E_{2}\right)$ has order 4 .

We have $\left|L_{K}\right|=q^{3}(q-1) /(q-1,3)$ and so, as $q>4, L_{K}$ is not a 2-group. Thus by Lemma $5.21 C_{G}\left(Y_{M}\right) C_{Q}(y)$ normalizes $K$ and so therefore does $Y_{M}$. By Lemma 3.10(ii) $Y_{M} \leq\left(S_{y} \cap K\right) C_{C_{S}(y)}(K)$. If $Y_{M} \not \leq Z(S \cap K) C_{C_{S}(y)}(K)$, then we may assume $Y_{M} \leq E_{1} C_{C_{S}(y)}(K)$ and $Y_{M} \not \leq E_{2} C_{C_{S}(y)}(K)$. Since $O_{2}(M)$ centralizes $Y_{M}$, we infer that $O_{2}(M)$ normalizes $E_{1}$. Hence $O_{2}(M)$ normalizes $N_{K}\left(E_{1}\right)$ and also $N_{K}\left(E_{2}\right)$. Then Lemma 4.8 implies that $Y_{M} \leq E_{2} C_{C_{S}(y)}(K)$, which is a contradiction. Hence

(10.4.1) $Y_{M} \leq Z\left(S_{y} \cap K\right) C_{C_{S}(y)}(K)$.

Since $\left[Z\left(S_{y} \cap K\right), L_{K}\right]=1$, we now have $O^{2}\left(L_{K}\right) \leq C_{G}\left(Y_{M}\right)$ and so, as $O_{2}(M)$ normalizes $L_{K}, K=E\left(C_{G}(y)\right)$ and $M^{\circ}$ normalizes $L_{K}=$ $O^{2}\left(L_{K}\right)$ by Lemma 5.22. Therefore

(10.4.2) $E_{y}=K$ and $M^{\circ}$ normalizes $O_{2}\left(L_{K}\right)=S_{y}$.

By Lemma 3.12, $K$ satisfies the Sylow centralizer property (Definition 5.13) with respect to $C_{S}(y)$. Furthermore all elements in $Z\left(S_{y}\right)$ are $K$-conjugate and, as $Q$ normalizes $Z\left(S_{y}\right)$, they all have a centralizer which has characteristic 2 . Thus Lemma 5.15 yields that

$$
T_{y} \text { is elementary abelian. }
$$


Since $Y_{M} \leq Z\left(S_{y}\right) T_{y},\left[S_{y}, Y_{M}\right] \leq T_{y}$. As $Q \leq M^{\circ}$ normalizes $S_{y}$ by $(10.4 .2)$, Lemma 5.7 implies that $S_{y} \leq C_{S}\left(Y_{M}\right)=O_{2}(M)$. Combining Lemma 2.8 with Lemma 3.10 (iii) yields

(10.4.3) $J\left(O_{2}(M)\right)=S_{y} T_{y}=E_{1} E_{2} T_{y}$.

In particular, (10.4.3) implies $N_{K}\left(S_{y}\right) \leq N_{G}\left(J\left(O_{2}(M)\right)\right) \leq M^{\dagger}$ and so $N_{K}\left(S_{y}\right)$ normalizes $Y_{M}$. As $N_{K}\left(S_{y}\right)$ acts irreducibly on $Z\left(S_{y}\right),(10.4 .1)$ produces $Y_{M} T_{y}=Z\left(S_{y}\right) T_{y}$. Therefore $\left[T_{y} Z\left(S_{y}\right), O_{2}(M)\right] \leq T_{y}$. Because $T_{y} Z\left(S_{y}\right)=J\left(O_{2}(M)\right)$ is normalized by $S$, we obtain $\left[T_{y} Z\left(S_{y}\right), O_{2}(M)\right]=$ 1. Thus

(10.4.4) $Y_{M}=T_{y} Z\left(S_{y}\right)$.

Assume that some element in $S$ conjugates $T_{y} E_{1}$ to $T_{y} E_{2}$. Let $A$ be an elementary abelian normal subgroup of $S$ contained in $O_{2}(M)$. Then $\widetilde{A} \leq \widetilde{S_{y}}$ by Lemma 3.10 (ii). Therefore, as $S$ does not normalize $T_{y} E_{1}$,

$$
A \leq T_{y} E_{1} \cap T_{y} E_{2}=T_{y} Z\left(S_{y}\right)=Y_{M}
$$

by (10.4.4), Applying Lemma 4.11 provides a contradiction. Thus we have

(10.4.5) $S \leq N_{G}\left(T_{y} E_{1}\right)$.

By (10.4.3) and (10.4.5), $M \leq N_{G}\left(E_{1} T_{y}\right)$. Thus $N_{G}\left(E_{1} T_{y}\right) \leq M^{\dagger}$. As $N_{K}\left(E_{1}\right) \leq N_{G}\left(E_{1} T_{y}\right)$ does not normalize $Y_{M}$, this is impossible.

Lemma 10.5. We have $K / Z(K) \neq \operatorname{Sp}_{2 n}(q)$ with $n \geq 3$ and $q=2^{a}$.

Proof. We follow the notation from Lemma 3.5. Thus $Z\left(S_{y} \cap K\right)=$ $R_{1} R_{2}$, and, for $i=1,2, L_{i}=C_{K}\left(R_{i}\right)$. We have $\widetilde{z}$ is centralized by $L_{1} \cap L_{2}$. Thus $L_{K}$ contains a section isomorphic to $\operatorname{Sp}_{2 n-4}(q)$, which is not a 2-group as $n \geq 3$. Thus Lemma 5.21 yields $K$ is normalized by $C_{G}\left(Y_{M}\right) C_{Q}(y)$. Since $L_{1}$ and $L_{2}$ are not isomorphic, we have $L_{1}$ and $L_{2}$ are normalized by $\mathrm{O}_{2}(M)$. Lemmas 4.8 and 5.27 imply that

$$
Y_{M} C_{C_{S}(y)}(K) \leq O_{2}\left(N_{K}\left(R_{1}\right)\right) C_{C_{S}(y)}(K) \cap O_{2}\left(N_{K}\left(R_{2}\right)\right) C_{C_{S}(y)}(K) .
$$

Furthermore, for $i=1,2,\left\langle Y_{M}^{N_{K}\left(R_{i}\right)}\right\rangle$ is elementary abelian. If $Y_{M} \not \subset$ $Z\left(O_{2}\left(N_{K}\left(R_{2}\right)\right)\right) C_{C_{S}(y)}(K)$, then Lemma 3.5 (ii) implies that

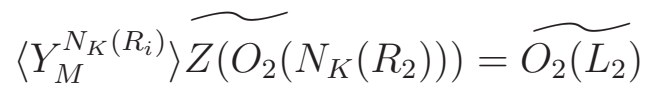

which is non-abelian. As $\left\langle Y_{M}^{N_{K}\left(R_{i}\right)}\right\rangle$ is abelian, we conclude

$$
\left.\widetilde{Y_{M}} \leq Z\left(\widetilde{O_{2}} \widetilde{\left(N_{K}\left(R_{2}\right)\right.}\right)\right)
$$


As $Z\left(\left(S_{y} \cap K\right) / Z(K)\right)=\left(Z\left(O_{2}\left(N_{K}\left(R_{2}\right)\right)\right) \cap O_{2}\left(N_{K}\left(R_{1}\right)\right)\right) / Z(K)$, we have that

$$
\widetilde{Y_{M}} \leq Z\left(\widetilde{S_{y} \cap K}\right)
$$

Now $Y_{M}$ is centralized by $O^{2}\left(L_{1} \cap L_{2}\right)$ and so Lemma 5.20 gives $K=E_{y}$ and $S_{y}=S_{y} \cap K$.

Assume that $Z(K) \neq 1$. Then, by [6, Table 6.1.3], $K / Z(K) \cong \operatorname{Sp}_{6}(2)$. Further the preimage of $Z\left(S_{y} / Z(K)\right)$ is isomorphic to $\mathbb{Z}_{4} \times \mathbb{Z}_{2}$. This contradicts Lemma 5.25. Hence $Z(K)=1$ and $K$ is simple.

Since $K=E_{y}$ is simple, $Y_{M} \leq Z\left(S_{y}\right) T_{y}$ which implies $\left[Y_{M}, S_{y}\right] \leq$ $K \cap T_{y}=1$. Thus $S_{y} \leq C_{S}\left(Y_{M}\right)=O_{2}(M)$. Using Lemmas 2.8 and 3.6 we obtain

$$
J\left(O_{2}(M)\right)=J\left(S_{y}\right) \times J\left(T_{y} \cap O_{2}(M)\right) .
$$

Therefore $N_{K}\left(J\left(S_{y}\right)\right) \leq N_{K}\left(J\left(O_{2}(M)\right)\right) \leq M^{\dagger}$. But $N_{K}\left(J\left(S_{y}\right)\right)$ normalizes no non-trivial subgroup in $Z\left(S_{y}\right)$ by Lemma 3.6, which is a contradiction as $Y_{M} \leq Z\left(S_{y}\right) T_{y}$ and $Y_{M} \not \leq T_{y}$. We have proved $K / Z(K) \varsubsetneqq \operatorname{Sp}_{2 n}(q)$ with $n \geq 3$ and $q=2^{a} \geq 2$.

Lemma 10.6. We have $K / Z(K) \nRightarrow \mathrm{F}_{4}(q)$ with $q=2^{a}, a \geq 1$.

Proof. By [6, Table 6.1.3] we have $Z(K)=1$ unless $q=2$. We use Lemmas 3.15 and 3.16 for structural information about $K / Z(K)$. Let $R_{1}$ and $R_{2}$ be the preimages of root subgroups of $K$ in $S_{y} \cap K$. Thus $\left|R_{i}\right|=q$ unless $Z(K) \neq 1$ in which case they are elementary abelian of order 4 . We also let $Z$ be the preimage of $Z((S \cap K) / Z(K))$.

By Lemma 3.11, $\widetilde{z} \in \widetilde{Z}$ and, by Lemma [5.1, $K=[K, z]$. We have $L_{K} \geq I_{12}$ (using the notation of Lemma 3.16) and so $L_{K}$ is not a 2-group. By Lemma 5.21

(10.6.1) $C_{G}\left(Y_{M}\right) C_{Q}(y)$ normalizes $K$.

We next intend to show

(10.6.2) $\widetilde{Y_{M}} \leq \widetilde{Z}$.

Suppose that $\widetilde{Y_{M}} \cap \widetilde{R_{i}} \neq 1$ for some $i \in\{1,2\}$. Without loss of generality we assume that $i=1$. Let $w \in Y_{M}$ be such that $\widetilde{w} \in \widetilde{R}_{1}^{\#}$. Then $O^{2}\left(C_{K}\left(R_{1}\right)\right)$ centralizes $w$. Hence $O_{2}(M)$ normalizes $O^{2}\left(C_{K}\left(R_{1}\right)\right)$. It follows that $O_{2}(M)$ normalizes $N_{K}\left(R_{1}\right)$ and $N_{K}\left(R_{2}\right)$. By Lemma 5.27

$$
\widetilde{Y_{M}} \leq O_{2}\left(\widetilde{N_{K}\left(R_{1}\right)}\right) \cap O_{2}\left(\widetilde{N_{K}\left(R_{2}\right)}\right) \text {. }
$$

For $i=1,2$, set $W_{i}=\left\langle Y_{M}^{N_{K}\left(R_{i}\right)}\right\rangle$. Then $W_{1}$ and $W_{2}$ are elementary abelian and Lemma 3.15 gives $\left.\widetilde{W_{i}} \leq Z\left(\widetilde{O_{2}\left(\widetilde{N_{K}(}\left(R_{i}\right)\right.}\right)\right)$. By Lemma 3.16 (ii), $Z\left(O_{2}\left(N_{K}\left(R_{1}\right)\right)\right) \cap Z\left(O_{2}\left(N_{K}\left(R_{2}\right)\right)\right)=Z$. Therefore $\widetilde{Y_{M}} \leq \widetilde{Z}$ in this case. 
To complete the proof of (10.6.2) we assume that

$$
\widetilde{Y_{M}} \cap \widetilde{R_{1}}=\widetilde{Y_{M}} \cap \widetilde{R_{2}}=1
$$

Since $O_{2}(M)$ normalizes $I_{12}$, Lemma 5.27 implies that $\widetilde{Y_{M}} \leq \widetilde{O_{2}\left(I_{12}\right)} \leq$ $\widetilde{K}$. Thus $\widetilde{Y_{M}}$ normalizes $\left.O_{2} \widetilde{\left(N_{K}\left(R_{i}\right)\right.}\right)$ for $i=1,2$. If, for some $i$, we have $\left.\widetilde{Y_{M}} \cap O_{2}\left(\widetilde{N_{K}\left(R_{i}\right.}\right)\right) \not Z Z\left(O_{2}\left(\widetilde{N_{K}\left(R_{i}\right)}\right)\right)$, then $1 \neq\left[O_{2}\left(\widetilde{N_{K}\left(R_{i}\right)}\right), \widetilde{Y_{M}}\right] \leq$ $\widetilde{R_{i}} \cap \widetilde{Y_{M}}=1$, a contradiction. Hence $\left.\widetilde{Y_{M}} \cap O_{2} \widetilde{\left(N_{K}\left(R_{i}\right)\right.}\right) \leq Z\left(O_{2}\left(\widetilde{N_{K}\left(R_{i}\right)}\right)\right)$ and so Lemma 3.15 implies that first $\left.\widetilde{Y_{M}} \leq O_{2} \widetilde{\left(N_{K}\left(R_{i}\right)\right.}\right)$ for both $i=1,2$ and then that

$$
\widetilde{Y_{M}} \leq Z\left(O_{2}\left(\widetilde{N_{K}\left(R_{1}\right)}\right)\right) \cap Z\left(O_{2}\left(\widetilde{N_{K}\left(R_{2}\right)}\right)\right)=\widetilde{Z} .
$$

This completes the proof of (10.6.2).

Since $O^{2}\left(I_{12}\right)$ centralizes $Z$, we have $O^{2}\left(I_{12}\right) \leq C_{K}\left(Y_{M}\right) \leq C_{G}\left(Y_{M}\right)$. Hence $J=C_{K}\left(Y_{M}\right)$ is not a 2-group. Notice that $J=O^{2}\left(N_{K}\left(R_{1}\right)\right)$ or $J=O^{2}\left(N_{K}(Z(S \cap K))\right)$, Lemmas 3.15 and 3.16 show that the noncentral $O^{2}(J)$-chief factors in $O_{2}\left(O^{2}(J)\right) / \Phi\left(O_{2}\left(O^{2}(J)\right)\right)$ are pairwise non-isomorphic. In this way Lemma 5.22 provides a contradiction. This proves $K / Z(K) \neq \mathrm{F}_{4}(q)$.

Lemma 10.7. We have $K / Z(K) \neq{ }^{2} \mathrm{~F}_{4}(q)^{\prime}$ with $q=2^{a} \geq 2$.

Proof. Suppose false. By Lemma 7.1 we have $q>2$ and 6, Theorem 6.1.4] states $Z(K)=1$. Furthermore [6. Theorem 2.5.12] gives Out $(K)$ has odd order.

For the structure of the 2-local subgroups of $K$ we shall use Lemma 3.17 in the arguments that follow, without specific reference. In particular, we know $L_{K}$ is not a 2-group and so Lemma 5.21 yields

$$
C_{G}\left(Y_{M}\right) C_{Q}(y) \text { normalizes } K \text {. }
$$

We introduce some notation. Let $R=Z(S \cap K)$ and $W_{1}=Z_{2}\left(O_{2}\left(L_{K}\right)\right)$. Then $L_{K}=C_{K}(R),\left|W_{1}\right|=q^{5}$ and $W_{1} / R$ is the natural $L_{K} / O_{2}\left(L_{K}\right)$ module where $L_{K} / O_{2}\left(L_{K}\right) \cong{ }^{2} \mathrm{~B}_{2}(q)$. Put $W_{2}=C_{K}\left(W_{1}\right)$. Then $\left|W_{2}\right|=$ $q^{6}$ and $W_{1}=\Omega_{1}\left(W_{2}\right)$. Let $P$ be the maximal parabolic subgroup of $K$ containing $N_{K}(S \cap K)$ but not containing $L_{K}$.

Since $L_{K}$ and $P$ are normalized by $O_{2}(M)$, by Lemma 4.8 we have $Y_{M} \leq O_{2}\left(L_{K} O_{2}(M)\right) \cap O_{2}\left(P O_{2}(M)\right)$ and $\left\langle Y_{M}^{L_{K}}\right\rangle$ and $\left\langle Y_{M}^{P}\right\rangle$ are elementary abelian.

As $\widetilde{\left\langle Y_{M}^{L_{K}}\right\rangle}$ is elementary abelian and normal in $\widetilde{L}_{K}$, we have $\widetilde{Y_{M}} \leq$ $\widetilde{\left\langle Y_{M}^{L_{K}}\right\rangle} \leq \widetilde{W_{1}}$. If $\widetilde{Y_{M}}=\widetilde{W_{1}}$, then $\widetilde{\left\langle Y_{M}^{P}\right\rangle}$ is not abelian, a contradiction. 
Hence $\widetilde{Y_{M}}<\widetilde{W_{1}}$. In particular, any subgroup $W$ of $Y_{M}$ which is normalized by $O^{2}\left(L_{K}\right)$ has $\widetilde{W} \leq \widetilde{R}$ and so $W$ is centralized by $O^{2}\left(L_{K}\right)$. Lemma 5.21 implies that

$$
E\left(C_{G}(y)\right)=K \text { and } Q \text { normalizes } O^{2}\left(L_{K}\right) .
$$

In particular, some element of $R$ is centralized by $Q$. Since all root elements are conjugate, all the involutions in $R$ have characteristic 2 centralizers. Therefore Lemma 5.15 yields $T_{y}$ is elementary abelian of order at most $q$. Since $O_{2}(M) \leq S_{y} T_{y}$, and $T_{y} \leq Z\left(S_{y} T_{y}\right), T_{y} \leq Y_{M}$. Hence $Y_{M}=\left(Y_{M} \cap K\right) T_{y}$.

Assume that $\widetilde{Y_{M}} \leq \widetilde{R}$. Then $L_{K}=O^{2}\left(L_{K}\right) \leq C_{G}\left(Y_{M}\right)$. Hence Lemma 5.22 implies that

$$
L_{K}=O^{2}\left(L_{K}\right) \text { is normalized by } M^{\circ} \text {. }
$$

Considering the action of $Q$ on $V=O_{2}\left(L_{K}\right) / W_{1}$, which is an indecomposable 5-dimensional $\mathrm{GF}(q)$-module for $L_{K} / O_{2}\left(L_{K}\right)$, we see that $C_{V}(Q)$ has a non-trivial $O^{2}\left(L_{K}\right)$ chief factor by the $A \times B$-lemma. Hence $Q$ centralizes $V$. As $Z_{2}\left(O_{2}\left(L_{K}\right)\right)=\Phi\left(O_{2}\left(L_{K}\right)\right), O^{2}\left(M^{\circ}\right)$ centralizes $O_{2}\left(L_{K}\right)$ which is normalized by $Q$ and so this contradicts Lemma 4.5. Hence

$$
\widetilde{Y}_{M} \not \subset \widetilde{R} .
$$

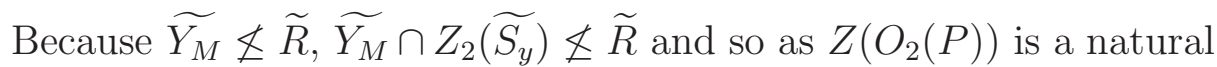
$P / O_{2}(P)$-module where $P / O_{2}(P) \cong \mathrm{SL}_{2}(q),\left[\widetilde{Y_{M}} \cap Z_{2}\left(\widetilde{S_{y}}\right), \widetilde{S_{y}}\right]=\widetilde{R}$ and we deduce $\left[S_{y}, Y_{M}\right] \geq R$. Since $O_{2}(M) P=C_{O_{2}(M) P}(K) P$ and $\widetilde{O_{2}(M)}$ centralizes $Y_{M} \cap Z_{2}\left(S_{y}\right)$ we conclude that $P$ normalizes $O_{2}(M) T_{y}$. But then $P$ normalizes $\mathrm{O}_{2}(M)$ as $\mathrm{O}_{2}(M)$ is weakly closed in $S$ by Lemma 4.4. In particular, $Y_{M} \cap K$ is normalized by $P$ and so $Y_{M} \cap K \geq Z_{2}\left(S_{y}\right)$.

Since $O_{2}\left(L_{K}\right) / C_{O_{2}\left(L_{K}\right)}\left(W_{1}\right)$ and $W_{1} / R$ are irreducible $O^{2}\left(L_{K}\right)$-modules, $Q \leq M^{\circ}$ normalizes $L_{K}$ and $L_{K} \leq C_{G}(z)$ normalizes $Q, Q$ centralizes these sections. Therefore

$$
\left[O_{2}\left(L_{K}\right), Q, W_{1}\right]=1
$$

and

$$
\left[W_{1}, Q, O_{2}\left(L_{K}\right)\right]=1
$$

The Three Subgroups Lemma implies that $\left[W_{1}, O_{2}\left(L_{K}\right), Q\right]=1$. Since $\left[W_{1}, O_{2}\left(L_{K}\right)\right]$ is normalized by $N_{K}(R)$ and $N_{K}(R)$ permutes the involutions in $R$ transitively, $R=\left[W_{1}, O_{2}\left(L_{K}\right)\right] \leq Z(Q)$. Let $\omega \in O^{2}\left(L_{K}\right)$ have order $q+\sqrt{2 q}+1$. Then $\omega$ acts fixed-point-freely on the natural module for $L_{K} / O_{2}\left(L_{K}\right) \cong{ }^{2} \mathrm{~B}_{2}(q)$. Since $\omega$ normalizes $Q$, we have 
$Q=[Q, \omega] C_{Q}(\omega)$ and $[Q, \omega] \leq L_{K}$. Now $C_{Q}(\omega)$ normalizes $\left[W_{1}, \omega\right]$ and as $\left[W_{1}, Q\right] \leq R=C_{W_{1}}(\omega)$, we have

$$
\left[\left[W_{1}, \omega\right], C_{Q}(\omega)\right] \leq\left[W_{1}, \omega\right] \cap C_{W_{1}}(\omega)=1 .
$$

Hence $C_{Q}(\omega)$ centralizes $W_{1}=R\left[W_{1}, \omega\right]$.

By Lemma 4.11, $O_{2}(M)$ is not abelian. Since $O_{2}(M) \leq S_{y} T_{y}$ and $T_{y}$ is abelian, we have $O_{2}(M)^{\prime} \leq S_{y}$ and $O_{2}(M)^{\prime} \cap Y_{M}$ is normalized by $P$. In particular,

$$
R<Z\left(O_{2}(P)\right) \leq O_{2}(M)^{\prime} \cap Y_{M} .
$$

Choose $x \in C_{Q}(\omega) \backslash C_{Q}(y)$ such that $x^{2} \in C_{Q}(y)$. Then

$$
x^{2} \in C_{S_{y} T_{y}}(\omega)=C_{S_{y}}(\omega) T_{y} \leq C_{S_{y} T_{y}}\left(W_{1} T_{y}\right) \leq C_{S_{y} T_{y}}\left(Y_{M}\right) \leq O_{2}(M) .
$$

We consider the action of $x$ on $Y_{M}$. As $x$ centralizes $W_{1}$ and $T_{y} \cap T_{y}^{x}=1$ by Lemma 5.10 and Lemma 5.9, $C_{Y_{M}}(x)=Y_{M} \cap W_{1}=Y_{M} \cap K$. As $x^{2} \in O_{2}(M)$,

$$
\left[Y_{M}, x\right] \leq C_{Y_{M}}(x)=Y_{M} \cap K .
$$

We also have $\left[O_{2}\left(L_{K}\right), x\right] \leq\left[O_{2}\left(L_{K}\right), Q\right] \leq C_{O_{2}\left(L_{K}\right)}\left(W_{1}\right)$ and so

$$
\left[\mathrm{O}_{2}\left(L_{K}\right), x, Y_{M}\right]=1 \text {. }
$$

As $\left[Y_{M}, O_{2}\left(L_{K}\right), x\right] \leq[R, x]=1$, the Three Subgroups Lemma implies that

$$
\left[Y_{M}, x\right] \leq C_{Y_{M}}\left(O_{2}\left(L_{K}\right)\right)=R \leq O_{2}(M)^{\prime} \cap Y_{M} \leq K \cap Y_{M}=C_{Y_{M}}(x) .
$$

Hence $x$ centralizes the sections of the $M$-invariant series

$$
Y_{M}>Y_{M} \cap O_{2}(M)^{\prime}>1
$$

which means that $x \in O_{2}\left(M / C_{M}\right)=1$. Thus $x$ centralizes $Y_{M}$. But then $x$ centralizes $y$ and this is impossible. Therefore $C_{Q}(\omega) \leq C_{Q}(y)$ and $Q=[Q, \omega] C_{Q}(y) \leq O_{2}\left(L_{K}\right) C_{Q}(y) \leq C_{G}(y)$, a contradiction.

Lemma 10.8. Suppose that $K / Z(K) \cong \operatorname{PSL}_{n}(q), n \geq 4$ and $q=2^{a}$. Then $K / Z(K) \cong \operatorname{PSL}_{3}(4)$.

Proof. Suppose false. By Lemmas 10.2 and 10.4, $n \geq 4$. Furthermore, as $\mathrm{PSL}_{4}(2) \cong \operatorname{Alt}(8)$, we have by Proposition 9.5 that $K / Z(K) \nsucceq$ $\mathrm{PSL}_{4}(2)$. Now by Lemmas 3.11 and 5.1 and [6, Theorem 6.1.4] we have $K$ is simple. Let $R=Z(S \cap K)$. Then Lemma 3.11 implies that $\widetilde{z} \in \widetilde{R}$. Thus $L_{K}$ is not a 2-group and so $K$ is normalized by $C_{G}\left(Y_{M}\right) C_{Q}(y)$ by Lemma 5.21. Since $(n, q) \neq(4,2)$, Lemmas 3.1 and 3.2 show that $O_{2}\left(L_{K}\right) / R$ is a direct sum of two non-isomorphic $C_{K}(R)$-modules. Let their preimages be $E_{1}$ and $E_{2}$. We have $E_{1}$ and $E_{2}$ are elementary abelian and they have order $q^{n-1}$.

If $Y_{M} \leq R C_{C_{S}(y)}(K)$, then $O^{2}\left(L_{K}\right)$ centralizes $Y_{M}$ and the aforementioned module structure of $\mathrm{O}_{2}\left(L_{K}\right)$ provides a contradiction via Lemma 
5.22 (ii). Therefore $Y_{M} \not \leq R C_{C_{S}(y)}(K)$. Since $U_{Q}$ is elementary abelian and is normalized by $L_{K}$ and $U_{Q} \not \leq R C_{C_{S}(y)}(K)$, we have, without loss of generality, $U_{Q} C_{C_{S}(y)}(K)=E_{1}$. Hence $O_{2}(M)$ normalizes $E_{1}$ and hence also $E_{2}$. But then $Y_{M} \leq O_{2}\left(O_{2}(M) N_{K}\left(E_{2}\right)\right) \leq E_{2} C_{C_{S}(y)}(K)$ by Lemma 5.27. Therefore $Y_{M} \leq E_{1} C_{C_{S}(y)}(K) \cap E_{2} C_{C_{S}(y)}(K)=R C_{C_{S}(y)}(K)$ and we have a contradiction.

Lemma 10.9. We have $K / Z(K) \varsubsetneqq \mathrm{G}_{2}(4)$.

Proof. By [6, Table 6.1.3] we have $|Z(K)| \leq 2$. Let $R$ be the preimage of $Z((S \cap K) / Z(K))$. By Lemma 3.11, $\widetilde{z} \in \widetilde{R}^{\#}$. As $U_{Q}$ is elementary abelian and normalized by $L_{K}$, application of Lemma 3.18 shows that $Y_{M} C_{S}(K)$ is centralized by $O^{2}\left(L_{K}\right)$. Therefore $K=E_{y}$ and $M^{\circ}$ normalizes $O^{2}\left(L_{K}\right)$ by Lemma 5.22. Since, by Lemma 5.25, $4 \leq\left|Y_{M} T_{y} / T_{y}\right| \leq$ $\left|Z(S \cap K) T_{Y} / T_{Y}\right| \leq 4$, we have $Y_{M} T_{y} / T_{Y}=Z(S \cap K) T_{Y} / T_{Y}$ has order 4. Lemmas 4.12 and 5.11 imply that

$$
\left|Y_{M}\right|=16 .
$$

Furthermore by Lemma 5.15 we have that

$$
T_{y}=Y_{M} \cap T_{y} .
$$

Notice that $O_{2}(M) \leq C_{S_{y} O_{2}(M)}(R / Z(K))$ and so $O_{2}(M)$ is normalized by $N_{K}(S \cap K)$. This subgroup contains an element $\rho$ of order 3 which operates non-trivially on $R / Z(K)$. It follows that $\left[Y_{M}, \rho\right]$ has order 4 and $\rho$ centralizes $C_{Y_{M}}(K)$. Since $z \in O_{2}(M)^{\prime} \leq K$ and we have $Y_{M} \cap O_{2}(M)^{\prime}$ has order 4 or 8 . Suppose first that the order is 8 . Then $1 \neq Z(K) \leq R$. Since $O_{2}\left(M^{\dagger} / C_{M}\right)=1$ and $M^{\dagger}$ is not transitive on $R$, we have $M^{\dagger} / C_{M} \cong \operatorname{Sym}(3)$ and $Z(K)=C_{R}(\rho)$ is centralized by $Q$, a contradiction. Hence $R=Y_{M} \cap O_{2}(M)^{\prime}$ has order 4. Since $\rho$ centralizes $Y_{M} / R$, we have $\langle\rho\rangle$ is normalized by $S$ and hence so is $C_{Y_{M}}(\rho)=$ $C_{Y_{M}}(K)$. But then $Z(Q) \cap T_{y} \neq 1$, and so we have a contradiction.

Proposition 10.10. If $K / Z(K)$ is a simple group of Lie type in characteristic 2 , then $K / Z(K) \cong \mathrm{PSL}_{3}(4)$ or $\operatorname{Sp}_{4}(q), q=2^{a} \geq 4$.

Proof. Suppose false and let $q=2^{a}$. Then combining the lemmas of this section, we have $K / Z(K) \cong \mathrm{U}_{n}(q), n \geq 4, \Omega_{2 n}^{ \pm}(q), n \geq 4$, $\mathrm{G}_{2}(q), q \geq 8,{ }^{3} \mathrm{D}_{4}(q),{ }^{2} \mathrm{E}_{6}(q)$ or $\mathrm{E}_{n}(q), n=6,7,8$. Also, by Proposition [8.5, $K / Z(K) \neq \mathrm{PSU}_{4}(2) \cong \mathrm{PSp}_{4}(3)$. Lemma 3.11 implies that $z$ acts on $K / Z(K)$ as a 2-central element. Set $L_{K}=C_{K}(z)$ and $J=$ $C_{O^{2}\left(L_{K}\right)}\left(Z\left(O_{2}\left(O^{2}\left(L_{K}\right)\right)\right)\right)$. Then Lemma 3.1 implies $O_{2}\left(O^{2}\left(L_{K}\right)\right)$ is nonabelian and $O^{2}(J)$ acts irreducibly on $O_{2}\left(O^{2}\left(L_{K}\right)\right) / Z\left(O_{2}\left(O^{2}\left(L_{K}\right)\right)\right)$. This contradicts Lemma 5.23 and proves the proposition. 


\section{The Groups $\mathrm{PSL}_{3}(4)$ And $\mathrm{PSp}_{4}(q)$ AS COMPONEnts}

In this section we assume that $K / Z(K) \cong \mathrm{PSL}_{3}(4)$ or $\operatorname{PSp}_{4}(q), q=$ $2^{a}>2$. We will show that this is not possible. By [6, Theorem 6.1.4] we have $Z(K)=1$ if $K \cong \operatorname{PSp}_{4}(q)$. By Lemma 3.11, $z$ acts as an inner automorphism on $K$ and so $Z(K)$ is a 2-group by Lemma 5.1

Lemma 11.1. If $K / Z(K) \cong \operatorname{PSL}_{3}(4)$ and $Z(K) \neq 1$, then $Z(K)$ is elementary abelian of order at most 4.

Proof. Assume that $Z(K)$ contains an element of order four. Then $Z(S \cap K)=Z(K)$ by Lemma 3.9 and $z$ acts on $K$ as an element of $Z(S \cap K)$ and so $z$ centralizes $K$. This contradicts Lemma 5.1. Thus $Z(K)$ is elementary abelian of order at most 4 by $[\underline{6}$, Theorem 6.1.4].

We now establish the notation which will be used throughout this section. We write

$$
E_{y}=K_{1} \ldots K_{r}
$$

where each $K_{i}$ is a component of $C_{G}(y)$ with $K_{i} / Z\left(K_{i}\right) \cong K / Z(K)$ and $\left|K_{i}\right|=|K|$. For $1 \leq i \leq r$, we define

$$
S_{i}=S_{y} \cap K_{i}
$$

If $K \cong \mathrm{PSp}_{4}(q)$, we let $E_{i j}, j=1,2$, be the maximal order elementary abelian subgroups of order $q^{3}$ in $S_{i}$ described in Lemma 3.7. If $K / Z(K) \cong \mathrm{PSL}_{3}(4)$, then we let $E_{i j}, j=1,2$, be the elementary abelian subgroups of order $16|Z(K)|$ as described in Lemma 3.13 (i). In all cases we have that every elementary abelian subgroup of $S_{i}$ is contained in $E_{i 1}$ or in $E_{i 2}$. When discussing a fixed component $K$, we often abbreviate our notation using $E_{1}$ and $E_{2}$ in place of $E_{i 1}$ and $E_{i 2}$.

Define

$$
D_{y}=J\left(O_{2}(M) \cap T_{y}\right) .
$$

The proof takes different directions depending upon whether or not $D_{y}$ is abelian.

Lemma 11.2. Suppose that $K / Z(K) \cong \operatorname{Sp}_{4}(q), q=2^{a} \geq 4$. Then $Z(K)=1$ and no element of $\Omega_{1}(Z(S))$ projects on to a root element of $\widetilde{K}$.

Proof. By [6, Table 6.1.3] we have $Z(K)=1$. Let $Z(S \cap K)=R_{1} R_{2}$ with $R_{1}$ and $R_{2}$ root subgroups. Suppose that $z \in \Omega_{1}(Z(S))$ is such that

$\widetilde{z}$ is a root element in $\widehat{S \cap K}$. Then $L_{K}$ is not a 2-group and so Lemma 5.21 implies $C_{G}\left(Y_{M}\right) C_{Q}(y)$ normalizes $K$ and then $C_{Q}(y)$ normalizes $O^{2}\left(L_{K}\right)$. 
Suppose $\left.\widetilde{Y_{M}}=O_{2}\left(\widetilde{N_{K}\left(R_{1}\right.}\right)\right)$. Then $O_{2}(M)$ normalizes $N_{K}\left(R_{1}\right)$ and $N_{K}\left(R_{2}\right)$ and $Y_{M} \not \leq O_{2}\left(N_{G}\left(R_{2}\right)\right)$. Employing Lemma 4.8 and Lemma 5.27 we have a contradiction. Now Lemma 3.7 shows that $O^{2}\left(L_{K}\right)$ centralizes any subgroup of $Y_{M}$, which is normalized by $O^{2}\left(L_{K}\right)$. Application of Lemma 5.20 shows that $Q$ normalizes $O^{2}\left(L_{K}\right)$. Then, as $\mathrm{O}_{2}\left(L_{K}\right)$ is elementary abelian and contains exactly one non-central $O^{2}\left(L_{K}\right)$-chief factor, Lemma 5.24 implies $O_{2}\left(L_{K}\right) \leq Z(Q)$. Hence $K=$ $\left\langle C_{K}\left(R_{1}\right), C_{K}\left(R_{2}\right)\right\rangle \leq N_{G}(Q)$, a contradiction. This proves the lemma.

We remark that the next lemma does not require that $\left|C_{S}(y)\right|$ is chosen to be maximal.

Lemma 11.3. Suppose that $y \in \mathcal{Y}_{S}^{*}$. Then the following hold.

(i) $N_{E_{y}}\left(S_{y}\right) \leq M^{\dagger}$.

(ii) $Y_{M}=\left(Y_{M} \cap T_{y}\right)\left(Y_{M} \cap S_{y}\right)$ and $\left(Y_{M} \cap K\right) Z(K)=Z\left(S_{y} \cap K\right)$.

(iii) $O_{2}(M)$ normalizes $K$ and $J\left(O_{2}(M)\right)=S_{y} D_{y}$.

(iv) Either $\mathrm{O}_{2}(M)=S_{y}\left(\mathrm{O}_{2}(M) \cap T_{y}\right)$ or $\mathrm{O}_{2}(M) K$ is isomorphic to $\mathrm{PSL}_{3}(4)$ extended by a graph automorphism.

Proof. By Lemma 5.16, $Y_{M}$ normalizes $K$. Thus $\left[S_{y} \cap K, Y_{M}\right] \leq Y_{M} \cap K$.

Assume that $\widetilde{Y_{M}} \cap \widetilde{K} \not Z Z\left(\widetilde{S_{y} \cap K}\right)$. Then $\left[Y_{M}, S_{y} \cap K\right] \not Z Z(K)$ and so $Y_{M} \cap K \not L Z(K)$. Therefore, as $\mathrm{O}_{2}(M)$ centralizes $Y_{M}, \mathrm{O}_{2}(M)$ normalizes $K$. We may assume that $\widetilde{Y_{M}} \cap \widetilde{S_{y} \cap K}$ is contained in $\widetilde{E_{1}}$ but not in $Z\left(\widehat{S_{y} \cap K}\right)$. In particular $\widetilde{O_{2}(M)}$ normalizes $\widetilde{E_{1}}$. But then $O_{2}(M)$ normalizes $E_{1}$ and also normalizes $E_{2}$. We have that $J=N_{K}\left(E_{2}\right)$ is of characteristic 2 and is normalized by $O_{2}(M)$. However $\widetilde{Y_{M}} \not \mathbb{E _ { 2 }}$ and this contradicts Lemma 4.8. Thus

(11.3.1) $\widetilde{Y_{M}} \cap \widetilde{S_{y} \cap K} \leq Z\left(\widetilde{S_{y} \cap K}\right)$.

Assume there exits $x \in Y_{M}^{\#}$, which induces an outer automorphism on $K$. Then $\left[\widetilde{x}, \widetilde{S_{y} \cap K}\right] \leq\left[\widetilde{Y_{M}}, \widetilde{S_{y} \cap K}\right] \leq \widetilde{Y_{M}} \cap \widehat{S_{y} \cap K} \leq Z\left(\widehat{S_{y} \cap K}\right)$. In particular $x$ cannot interchange $E_{1}$ and $E_{2}$. This yields that $x$ induces a field automorphism on $K$. But such an automorphism is non-trivial on $E_{1} / Z\left(S_{y} \cap K\right)$. Therefore $\widetilde{Y_{M}} \leq \widetilde{S_{y} \cap K}$ which then means by $(11.3 .1)$ (11.3.2) $\widetilde{Y_{M}} \leq Z\left(\widetilde{S_{y} \cap K}\right)$.

That is

$$
Y_{M} \leq Z\left(S_{y} \cap K\right) C_{C_{S}(y)}(K) \leq C_{C_{S}(y)}\left(S_{y} \cap K\right)
$$


Since this is true for all the components of $E_{y}$, we have

(11.3.3) $S_{y} \leq C_{S}\left(Y_{M}\right)=O_{2}(M)$.

Consider $\bar{E}_{y}=E_{y} O_{2}(M) / Z\left(E_{y}\right)$. Then $\overline{O_{2}(M)}$ is a Sylow 2-subgroup of $\overline{E_{y}}$. We have $\overline{E_{1} E_{2}}=J\left(\overline{S_{y} \cap K}\right)=J\left(\overline{O_{2}(M)}\right)$ by Lemma 3.13 and Lemma 3.7. Therefore, by Proposition 2.7 and Lemmas 3.14 and 11.1 we get $J\left(O_{2}(M)\right)$ normalizes $K$. It follows that $J\left(O_{2}(M)\right) \cap E_{y}=S_{y}$ and $J\left(O_{2}(M)\right)=S_{y} D_{y}$ by Lemma 2.8 and the definition of $D_{y}$. In particular, $J\left(O_{2}(M)\right)$ is normalized by $N_{E_{y}}\left(S_{y}\right)$ and so $N_{E_{y}}\left(S_{y}\right) \leq M^{\dagger}$ by Lemma 4.3. Hence (i) is true.

Using (i) and the fact that $1 \neq \widetilde{Y_{M}} \leq Z\left(\widetilde{S_{y} \cap K}\right)$ by $(11.3 .2)$, we have $\widetilde{Y_{M}}=Z\left(\widetilde{S_{y} \cap K}\right)$, as by Lemma 11.2 $\widetilde{Y}_{M}$ is not contained in a root group when $K \cong \operatorname{Sp}_{4}(q)$. Further

$$
1 \neq\left[Y_{M}, N_{K}\left(S_{y} \cap K\right)\right] Z(K) / Z(K)=Z((S \cap K) / Z(K)) .
$$

In particular, $Y_{M} \cap K \not \leq Z(K)$ and so $O_{2}(M)$ normalizes $K$. Furthermore, letting $C$ be a complement to $S_{y}$ in $N_{E_{y}}\left(S_{y}\right)$, we have

$$
Y_{M}=\left[Y_{M}, C\right] C_{Y_{M}}(C)=\left(Y_{M} \cap S_{y}\right)\left(Y_{M} \cap T_{y}\right)
$$

and $\left[Y_{M}, C\right] Z(K)=Z\left(S_{y}\right)$. This is (ii).

We have just seen that $O_{2}(M)$ normalizes all the components of $E_{y}$ and $S_{y} \leq O_{2}(M)$. We have also proved $J\left(O_{2}(M)\right)=S_{y} D_{y}$. This is (iii).

Since $O_{2}(M)$ normalizes $K$ and $\widetilde{O_{2}(M)}$ centralizes $Z\left(\widetilde{S_{y} \cap K}\right)$ by (ii), either $\widetilde{O_{2}(M)}=\widetilde{S_{y}}$ or $\widetilde{O_{2}(M)} K$ is isomorphic to $\operatorname{PSL}_{3}(4)$ extended by a graph automorphism (see [7, Chapter 10, Lemma 2.1]). Hence (iv) holds.

Lemma 11.4. Suppose that $D_{y}$ is abelian and $r \geq 2$. If $x \in\left(Y_{M} \cap\right.$ $\left.K_{i}\right) \backslash Z\left(K_{i}\right)$ for some $1 \leq i \leq r$, then $E_{x}=\prod_{j \neq i} K_{j}<E_{y}$.

Proof. By Lemma 5.5, $\prod_{j \neq i} K_{j} \leq E_{x}$ and $\prod_{j \neq i} K_{j}$ is non-trivial as $r \geq 2$. Assume that $E_{x}>\prod_{j \neq i} K_{j}$. Then there is a component $L$ of $C_{G}(x)$ with $L / Z(L) \cong K / Z(K),|L|=|K|$ and $L \not \leq \prod_{j \neq i} K_{j}$. By Lemma 5.5, the normal closure of $\prod_{j \neq i} K_{j}$ in $E_{x}$ has at least $r-1$ components of $C_{G}(x)$ and only has $r-1$ components if every component of $\prod_{j \neq i} K_{j}$ is a component of $C_{G}(x)$. Therefore $E_{x}$ has exactly $r$ components.

Suppose that $K$ is simple. Then $E_{x} \cong E_{y}$ and we can apply Lemma 11.3 to find $S_{x} \cap L=O_{2}(M) \cap L \in \operatorname{Syl}_{2}(L)$ and $S_{x} \cap L \leq J\left(O_{2}(M)\right)=$ $S_{y} D_{y}$. Consider $K_{j} \leq E_{x}$ and let $C$ be a complement to $N_{K_{j}}\left(S_{j}\right)$. Then 
$\left[S_{y} D_{y}, C\right]=S_{j}$ and so, as $C$ normalizes $L$,

$$
\left[S_{x} \cap L, C\right] \leq S_{j} \cap L \leq K_{j} \cap L \leq Z\left(K_{j}\right)=1 .
$$

Hence, temporarily setting $\overline{E_{x}}=E_{x} / C_{E_{x}}(L)$, we have

$$
\bar{C} \leq C_{\bar{L}}\left(\overline{S_{x} \cap L}\right)=Z\left(\overline{S_{x} \cap L}\right)
$$

and this means that $C \leq C_{E_{x}}(L)$. Hence $K_{j} \cap C_{E_{x}}(L) \not \leq Z\left(K_{j}\right)$ which means that $K_{j}$ centralizes $L$. Therefore $L$ centralizes $\prod_{j \neq i} K_{j}$ and so

$$
S_{x} \cap L \leq C_{J\left(O_{2}(M)\right)}\left(\prod_{j \neq i} K_{j}\right)=D_{y} S_{i}
$$

Since $D_{y}$ is abelian, this shows that $(S \cap L)^{\prime}=S_{i}^{\prime}$. As $x \in S_{i}^{\prime} \leq L$ and $x$ centralizes $L$, we deduce $Z(L) \neq 1$, a contradiction. Hence

$$
Z(K) \neq 1 \text {. }
$$

As $O_{2}(M)$ normalizes $K_{j}, 1 \leq j \leq r$, by Lemma 11.3 (iii), we can choose an involution $w \in Z\left(\prod_{j \neq i} K_{j}\right) \cap Y_{M}$. Then $w \in D_{y}$ and $E_{w}=E_{y}$ by Lemma 5.6. Since $L$ is a component of $C_{G}(x)$ and $K_{j}$ is quasisimple for $2 \leq j \leq r$, we have

$$
L / Z(L) \cong L C_{E_{x}}(L) / C_{E_{x}}(L) \cong K_{j} C_{E_{x}}(L) / C_{E_{x}}(L) \cong K_{j} / Z\left(K_{j}\right)
$$

and so $\prod_{i \neq j} Z\left(K_{j}\right) \leq C_{E_{x}}(L)$. It follows that $L$ centralizes $w$ and so $L$ normalizes $E_{w}=E_{y}$. Thus $L$ normalizes $E\left(C_{E_{y}}(x)\right)=\prod_{j \neq i} K_{j}$ and so $L$ normalizes $K_{i}=E\left(C_{E_{y}}\left(\prod_{j \neq i} K_{j}\right)\right)$. Since $L$ normalizes $\prod_{j \neq i} K_{j}$ and $L$ is a component in $E_{x}, L$ centralizes $\prod_{j \neq i} K_{j}$. Because $L$ centralizes $x$ and $L$ is a component of $C_{G}(x), L$ centralizes $C_{K_{i}}(x)$. We know that $C_{K_{i}}(x)=S_{i}$. Hence $L$ centralizes $S_{i} \prod_{j \neq i} K_{j} \geq S_{y}$. From Lemma 11.3(iii), $J\left(O_{2}(M)\right)=D_{y} S_{y}$. Therefore, as $D_{y}$ is abelian, $1 \neq$ $J\left(O_{2}(M)\right)^{\prime}=S_{y}^{\prime}$. Thus $L$ is a component in $C_{G}\left(J\left(O_{2}(M)\right)^{\prime}\right)$ and this contradicts Lemma 4.7 (ii). Thus $E_{x}=\prod_{j \neq i} K_{j}$, a claimed

Lemma 11.5. $D_{y}$ is non-abelian.

Proof. Assume that $D_{y}$ is abelian. By Lemma 11.3 (ii), $J\left(O_{2}(M)\right)=$ $D_{y} S_{y}$. As $D_{y}=J\left(D_{y}\right), D_{y}$ is elementary abelian. Now $\Omega_{1}\left(Z\left(J\left(O_{2}(M)\right)\right)\right)=$ $D_{y} Z\left(S_{y} \cap K\right)=D_{y} Y_{M}$ by Lemma 11.3(ii). Hence $\left[D_{y} Y_{M}, O_{2}(M)\right] \leq D_{y}$ and as $Z(S) \cap D_{y}=1$, we see that $\left[D_{y}, O_{2}(M)\right]=1$. Hence

$$
D_{y}=Y_{M} \cap T_{y}=\Omega_{1}\left(T_{y}\right) .
$$

Recall that $S_{i}$ contains exactly two maximal rank elementary abelian subgroups $E_{i 1}, E_{i 2}$ of order $16\left|Z\left(K_{i}\right)\right|$ if $K / Z(K) \cong \operatorname{PSL}_{3}(4)$, and $q^{3}$ 
otherwise. Thus the set of maximal order elementary abelian subgroups in $D_{y} S_{y}$ is

$$
\mathfrak{A}=\left\{D_{y} \prod_{i=1}^{r} E_{i i_{j}} \mid i_{j} \in\{1,2\}\right\}
$$

and $M$ permutes $\mathfrak{A}$ by conjugation. The group $M$ also permutes the pairs $\left(F_{1}, F_{2}\right) \in \mathfrak{A} \times \mathfrak{A}$ which have the property that

$$
\left|F_{1}: F_{1} \cap F_{2}\right|=\left|F_{2}: F_{1} \cap F_{2}\right|=\left\{\begin{array}{ll}
4 & K / Z(K) \cong \mathrm{PSL}_{3}(4) \\
q & \text { otherwise }
\end{array} .\right.
$$

Then $M$ permutes the set of commutators $\left[F_{1}, F_{2}\right]$ for all such pairs $\left(F_{1}, F_{2}\right)$. Let the set of such commutators be $\Theta$. Then, as $\left[E_{i 1}, E_{i 2}\right]=S_{i}^{\prime}$,

$$
\Theta=\left\{S_{i}^{\prime} \mid 1 \leq i \leq r\right\}
$$

and we have explained that

$$
M \text { permutes the groups in } \Theta \text {. }
$$

Assume that $r>1$. Then, as $M$ permutes $\Theta, M$ normalizes

$$
\begin{array}{ll}
N=\langle L| & L \text { a component of } C_{G}\left(Y_{M} \cap K_{i}\right), \\
& L / Z(L) \cong K / Z(K) \text { and }|L|=|K|, i=1, \ldots, r\rangle .
\end{array}
$$

By Lemmas 11.3 and 11.4, $N=\left\langle\prod_{j \neq i} K_{j} \mid 1 \leq i \leq r\right\rangle=E_{y}$ is normalized by $M$. It follows that $D_{y}=C_{J\left(O_{2}(M)\right)}\left(E_{y}\right)$ is normalized by $M$ and this contradicts Lemma 5.7 as $y \in D_{y}$ shows that $D_{y} \neq 1$. Thus

$$
r=1 \text { and } \mathfrak{A}=\left\{D_{y} E_{11}, D_{y} E_{12}\right\} .
$$

If $D_{y} E_{11}$ is normal in $M$, then $N_{K}\left(D_{y} E_{11}\right)=N_{K}\left(E_{11}\right) \leq M^{\dagger}$, but by Lemma 11.3 $Y_{M}$ is not normal in $N_{K}\left(E_{11}\right)$. Hence $D_{y} E_{11}$ and $D_{y} E_{12}$ are conjugate in $M$ and so in $S$. Suppose that $A \leq O_{2}(M)$ is an elementary abelian normal subgroup of $S$. By Lemma 11.3, we either have $O_{2}(M)=$ $\left(O_{2}(M) \cap T_{y}\right) J\left(O_{2}(M)\right)$ or $K / Z(K) \cong \mathrm{PSL}_{3}(4)$ and $O_{2}(M)$ induces a graph automorphism. In the latter case, Lemma 3.13 implies $A \leq$ $S_{y} T_{y}=J\left(O_{2}(M)\right)$. Hence always $A \leq \Omega_{1}\left(T_{y} S_{y}\right)=J\left(O_{2}(M)\right)$, and so, as $A$ is normal in $S$, we obtain $A \leq D_{y} E_{11} \cap D_{y} E_{12}=D_{y}\left(E_{11} \cap E_{12}\right)=$ $Y_{M}$, as $D_{y}=Y_{M} \cap T_{y}$. This contradicts Lemma 4.11,

Proposition 11.6. Suppose that $y \in \mathcal{Y}_{S}^{*}$ and let $K \leq E_{y}$ be a component of $C_{G}(y)$. Then $K / Z(K) \neq \operatorname{PSL}_{3}(4)$ or $\operatorname{Sp}_{4}(q), q=2^{a}>2$.

Proof. Assume the proposition is false. By Lemma $11.5 D_{y}$ is nonabelian. We first prove the following claim. 
(11.6.1) The component $K$ is simple and there exists $N \leq G$ normalized by $M$ such that

$$
N=E(N)=E_{y} K_{r+1}=K_{1} \cdots K_{r+1}
$$

with $\left[E_{y}, K_{r+1}\right]=1$ and $K \cong K_{r+1}$. Furthermore, $S \cap N=J\left(O_{2}(M)\right)$, $S$ permutes the components of $N$ transitively by conjugation, and $M=$ $S N_{M}\left(K_{1}\right)$.

Let $g \in N_{S}\left(N_{S}\left(T_{y}\right)\right) \backslash N_{S}\left(T_{y}\right)$ with $g^{2} \in N_{S}\left(T_{y}\right)$. By Lemma 5.10 , $D_{y} \cap D_{y}^{g}=1$ and $\left[D_{y}, D_{y}^{g}\right]=1$. As $D_{y} \leq J\left(O_{2}(M)\right)$ and $g$ normalizes $O_{2}(M), D_{y}^{g} \leq J\left(O_{2}(M)\right)$. As $D_{y}$ is normal in $N_{S}\left(T_{y}\right)$ the same applies for $D_{y}^{g}$.

For $1 \leq i \leq r$, set

$$
C_{i}=\prod_{j \neq i} S_{j} D_{y} .
$$

As, for $i \neq j, S_{i} \cap S_{j} \leq K_{i} \cap K_{j} \leq Z\left(K_{i}\right) \cap Z\left(K_{j}\right) \leq D_{y}$, the Modular Law implies $\bigcap_{i=1}^{r} C_{i}=D_{y}$. In addition, we also have $\left[S_{i}, C_{i}\right] \leq$ $\left[K_{i}, C_{i}\right]=1$.

If $D_{y}^{g} C_{i} / C_{i}$ is abelian for all $i$, then $\left(D_{y}^{g}\right)^{\prime} \leq \bigcap_{i=1}^{r} C_{i}=D_{y}$ contrary to $\left(D_{y}^{g}\right)^{\prime} \neq 1$ and $D_{y} \cap D_{y}^{g}=1$. Thus we may fix notation so that $D_{y}^{g} C_{1} / C_{1}$ is not abelian. Set $\overline{S_{y} D_{y}}=S_{y} D_{y} / C_{1}$. Then $\overline{D_{y}^{g}} \leq \overline{S_{1}}$ and $\overline{D_{y}^{g}} \not \overline{E_{1 j}}$ for $j=1,2$ as $\overline{D_{y}^{g}}$ is not abelian. Let $\rho \in N_{K_{1}}\left(S_{1}\right)$ be arbitrary of maximal odd order and such that $\rho$ acts fixed-point-freely on $S_{1} / Z\left(S_{1}\right)$. By Lemma $11.3 \rho \in N_{M^{\dagger}}\left(O_{2}(M)\right)$ and by Lemma 5.10 $T_{y}^{g} \cap O_{2}(M)$ is a trivial intersection group in $N_{G}\left(O_{2}(M)\right)$. As $D_{y}^{g}=$ $J\left(O_{2}(M) \cap T_{y}^{g}\right)$, we see that $\rho$ normalizes $D_{y}^{g}$ if and only if it normalizes $T_{y}^{g} \cap O_{2}(M)$. Suppose that $\rho$ does not normalize $D_{y}^{g}$. Then $\left[D_{y}^{g}, D_{y}^{g \rho}\right]=1$, as both groups are normal in $O_{2}(M)$. But then $\left[\overline{D_{y}^{g}}, \overline{D_{y}^{g \rho}}\right]=1$, and this contradicts Lemmas 3.8 and 3.13 (iii). Hence $\rho$ normalizes $D_{y}^{g}$. It also normalizes $\overline{D_{y}^{g}}$ and, as $D_{y}=J\left(D_{y}\right)$ is generated by involutions, it follows that $\overline{D_{y}^{g}}=\overline{S_{1}}$. In particular, $\left[S_{1}, D_{y}^{g}\right]=S_{1}^{\prime}=Z\left(S_{1}\right)$. We have $D_{y}^{g}=C_{D_{y}^{g}}(\rho)\left[D_{y}^{g}, \rho\right]$. Now $\left[S_{y} D_{y}, \rho\right]=S_{1}$. Hence $\left[D_{y}^{g}, \rho\right] \leq S_{1}$ and $C_{D_{y}^{g}}(\rho) \leq Z\left(S_{1}\right) C_{1}$. We conclude that $S_{1} \leq D_{y}^{g}$ as

$\overline{S_{1}}=\overline{D_{y}^{g}}=\overline{\left[D_{y}^{g}, \rho\right] C_{D_{y}^{g}(\rho)}} \leq \overline{\left[D_{y}^{g}, \rho\right] Z\left(S_{1}\right)} \leq \overline{\left[D_{y}^{g}, \rho\right]\left(S_{1} \cap D_{y}^{g}\right)} \leq \overline{\left(D_{y}^{g} \cap S_{1}\right)}$.

If $K_{1}$ is not simple, then $Z\left(K_{1}\right) \leq S_{1} \leq D_{y}^{g}$ and so $D_{y} \cap D_{y}^{g} \neq 1$, a contradiction. Hence the components in $E_{y}$ are simple groups. This proves the first statement in (11.6.1),

Since $D_{y}^{g} \geq S_{1}$ and $Y_{M} \cap S_{1}=Z\left(S_{1}\right)$ by Lemma 11.3, using Lemma 5.6 we have $E_{x}=E_{y}^{g}$ for all $x \in\left(Y_{M} \cap S_{1}\right)^{\#}$. 
If $K_{1} \cong \operatorname{Sp}_{4}(q)$, then $Z\left(S_{1}\right)$ contains root subgroups $R_{1}$ and $R_{2}$ and so $K_{1}=\left\langle C_{K_{1}}\left(R_{1}\right), C_{K_{1}}\left(R_{2}\right)\right\rangle$ normalizes $E_{y}^{g}$.

Suppose that $K_{1} \cong \mathrm{PSL}_{3}(4)$. Then all the involutions in $K_{1}$ are $K_{1^{-}}$ conjugate. Thus for involutions $t \in S_{1} \backslash Z\left(S_{1}\right)$, the group $E_{t}$ is conjugate to $E_{x}$. Since $t \in D_{y}^{g}$, we get $E_{t}=E_{y}^{g}$ by Lemma 5.4. Hence this time we see that $K_{1}=\left\langle C_{K}(t) \mid t \in S_{1}\right\rangle$ normalizes $E_{y}^{g}$. Thus in all cases

$$
K_{1} \text { normalizes } E_{y}^{g} \text {. }
$$

Furthermore, by Lemma [5.5, $K_{2} \ldots K_{r} \leq E_{y}^{g}$ (this is obviously true if $r=1)$. Hence

$$
E_{y} \text { normalizes } E_{y}^{g} \text {. }
$$

Since $g^{2} \in N_{G}\left(E_{y}\right)$, we also have $E_{y}^{g}$ normalizes $E_{y}^{g^{2}}=E_{y}$. It follows that the components of $E_{y}$ and the components of $E_{y}^{g}$ are components of $E_{y} E_{y}^{g}$. It now follows that $E_{y} \cap E_{y}^{g}=K_{2} \cdots K_{r}$ and that we can write

$$
N=E_{y} E_{y}^{g}=K_{1} K_{2} \cdots K_{r} K_{r+1}
$$

where $E_{y}^{g}=K_{2} \ldots K_{r+1}$.

We have $J\left(O_{2}(M)\right)=S_{y}^{g} D_{y}^{g}$ and so $S_{y}^{g} D_{y}^{g} \cap N \in \operatorname{Syl}_{2}(N)$. Furthermore $C_{O_{2}(M)}(N)=1$, as otherwise $C_{Y_{M}}(N) \neq 1$, but this is not possible as $y \in \mathcal{Y}^{*}$. Therefore

$$
J\left(O_{2}(M)\right) \in \operatorname{Syl}_{2}(N) .
$$

This verifies the second and third statement in (11.6.1).

Define $S_{r+1}=O_{2}(M) \cap K_{r+1}$. Then

$$
\prod_{i=1}^{r+1} S_{i} \in \operatorname{Syl}_{2}(N)
$$

We now argue as in the case when $D_{y}$ was abelian. The subgroups $S_{i}$ contains exactly two maximal rank elementary abelian subgroups $E_{i 1}, E_{i 2}$ of order 16 if $K \cong \operatorname{PSL}_{3}(4)$, and $q^{3}$ otherwise. Thus the set of maximal order elementary abelian subgroups in $J\left(O_{2}(M)\right)$ is

$$
\mathfrak{A}=\left\{\prod_{i=1}^{r+1} E_{i i_{j}} \mid i_{j} \in\{1,2\}\right\}
$$

and $M$ permutes $\mathfrak{A}$ by conjugation. The subgroup $M$ also permutes the pairs $\left(F_{1}, F_{2}\right) \in \mathfrak{A} \times \mathfrak{A}$ which have the property that

$$
\left|F_{1}: F_{1} \cap F_{2}\right|=\left|F_{2}: F_{1} \cap F_{2}\right|=\left\{\begin{array}{ll}
4 & K \cong \operatorname{PSL}_{4}(3) \\
q & \text { otherwise }
\end{array} .\right.
$$


Thus $M$ permutes the set of commutators $\left[F_{1}, F_{2}\right]$ for all such pairs $\left(F_{1}, F_{2}\right)$. As $\left[F_{1}, F_{2}\right]=Y_{M} \cap S_{i}$ for some $i, M$ permutates the set

$$
\Theta=\left\{Y_{M} \cap S_{i} \mid 1 \leq i \leq r\right\} .
$$

Hence $M$ normalizes the subgroup

$$
\begin{aligned}
N^{*}=\langle L| & L \text { a component of } C_{G}\left(Y_{M} \cap K_{i}\right), \\
& L / Z(L) \cong K / Z(K) \text { and }|L|=|K|, i=1, \ldots, r\rangle .
\end{aligned}
$$

Using the structure of $N$, we see that $N^{*}=N$. Hence $M$ normalizes $N$. In particular, $S$ permutes the set $\left\{K_{1}, \ldots K_{r+1}\right\}$ by conjugation. Suppose that $\left\{K_{j} \mid j \in J\right\}$ is an $S$-orbit. We get that $Z(S) \cap Y_{M} \cap \prod_{j \in J} K_{j} \neq$ 1. Application of Lemma $4.7(\mathrm{v})$ gives $J=\{1, \ldots, r+1\}$. Thus $S$ acts transitively on $\left\{K_{i} \mid 1 \leq i \leq r+1\right\}$. Finally, as $S$ is transitive on $\left\{K_{i} \mid 1 \leq i \leq r+1\right\}, M=N_{M}\left(K_{1}\right) S$ by the Frattini Argument. This completes the explanation of (11.6.1).

By (11.6.1), $M=N_{M}\left(K_{1}\right) S$ and so $S \cap N_{M}\left(K_{1}\right) \in \operatorname{Syl}_{2}\left(N_{M}\left(K_{1}\right)\right)$. We also know $N_{M}\left(K_{1}\right)$ normalizes $J\left(O_{2}(M)\right) \cap K_{1}=S_{1}$. Suppose that $E_{11}$ is not conjugate to $E_{12}$ in $N_{M}\left(K_{1}\right)$. Then $E_{11}$ is normal in $N_{M}\left(K_{1}\right)$ and $F=\left\langle E_{11}^{M}\right\rangle$ is elementary abelian with $F \cap K_{j} \in\left\{E_{j 1}, E_{j 2}\right\}$. Thus $F$ is normalized by $\left\langle M, N_{K_{1}}\left(E_{11}\right)\right\rangle \leq M^{\dagger}$. Since $N_{K_{1}}\left(E_{11}\right)$ does not normalize $Y_{M}$, this is impossible. Hence $E_{11}$ is conjugate to $E_{12}$ in $N_{M}\left(K_{1}\right)$. Since $S \cap N_{M}\left(K_{1}\right) \in \operatorname{Syl}_{2}\left(N_{M}\left(K_{1}\right)\right)$, (11.6.1) implies $S$ acts transitively on $\left\{E_{i j} \mid 1 \leq i \leq r+1, j=1,2\right\}$.

Let $A$ be an elementary abelian normal subgroup of $S$ contained in $O_{2}(M)$. Put $\overline{N_{M}\left(K_{1}\right)}=N_{M}\left(K_{1}\right) / C_{M}\left(K_{1}\right)$. Then $\overline{N_{M}\left(K_{1}\right)}$ normalizes $\overline{J\left(O_{2}(M)\right) \cap K_{1}}=\overline{S_{1}}$ and $\bar{A}$ is normal in $\overline{S \cap N_{M}\left(K_{1}\right)}$. It follows that

$$
\bar{A} \leq \overline{E_{11}} \cap \overline{E_{12}}=\overline{Y_{M}} \text {. }
$$

Hence $\left[A, O_{2}(M)\right] \leq C_{M}\left(K_{1}\right)$. Since $S$ acts transitively on $\left\{K_{1}, \ldots, K_{r+1}\right\}$ and $S$ normalizes $\left[A, O_{2}(M)\right]$, we have

$$
\left[A, O_{2}(M)\right] \leq O_{2}(M) \cap \bigcap_{i=1}^{r+1} C_{M}\left(K_{i}\right)=C_{O_{2}(M)}(N)=1 .
$$

Hence $A \leq Y_{M}$. Now application of Lemma 4.11 yields the contradiction. This proves the proposition.

\section{Proof of the Theorem}

Let $M$ and $Y_{M}$ be as in the assumption of the theorem. That is $Y_{M}$ is tall, asymmetric but not characteristic 2-tall. By Lemma 5.2 there is some $y \in Y_{M}^{\#}$ with $E\left(C_{G}(y)\right) \neq 1$. In particular $\mathcal{Y}_{S}^{*} \neq \emptyset$. For $y \in \mathcal{Y}_{S}^{*}$ we 
have $E_{y} \neq 1$. Let $K$ be a component of $E_{y}$. By Proposition $7.2 K / Z(K)$ is not a sporadic simple group. By Proposition 8.5 and Lemma 10.2 $K / Z(K)$ is not a group of Lie type in odd characteristic. Proposition 9.5 states that $K / Z(K)$ is not an alternating group. Hence $K / Z(K)$ a group of Lie type in characteristic 2. Proposition 10.10 shows that $K / Z(K) \cong \mathrm{PSL}_{3}(4)$ or $\operatorname{Sp}_{4}(q), q \geq 4$. Finally Proposition 11.6 provides the contradiction which proves the theorem.

\section{ACKNOWLEDGMENTS}

We are indebted to an anonymous referee for valuable suggestions which helped to improve the clarity and accuracy of the work presented here. In particular, the proof of Lemma 4.7 (i) was provided by the referee. The second author was partially supported by the DFG.

\section{REFERENCES}

[1] M.Aschbacher, B. Oliver, "Fusion systems", Bulletin of the AMS 53, (2016), 555-615.

[2] M. Aschbacher, "On fusion systems of component type", Mem. Amer. Math. Soc. 257 (2019).

[3] M. Aschbacher, G. Seitz, "Involutions in Chevalley groups over fields of even order", Nagoya Math. J. 63 (1976), 1-91; Correction, Nagoya Math. J. 72 (1978), 135-136.

[4] A. Delgado, D. Goldschmidt, B. Stellmacher, "Groups and Graphs: New Results and Methods", DMV Seminar 6 (1985), Birkhäuser.

[5] D. Gorenstein, R. Lyons, R. Solomon, "The Classification of the Finite Simple Groups Number 2", American Mathematical Society 40, \#2 (1996).

[6] D. Gorenstein, R. Lyons, R. Solomon, "The Classification of the Finite Simple Groups Number 3", American Mathematical Society 40, \#3 (1998).

[7] D. Gorenstein, R. Lyons, R. Solomon, "The Classification of the Finite Simple Groups Number 6", American Mathematical Society 40, \#6 (2004).

[8] B. Huppert. "Endliche Gruppen I", Springer Verlag, 1967.

[9] M. Mainardis, U. Meierfrankenfeld, G. Parmeggiani, B. Stellmacher, "The P !Theorem", Journal of Algebra 292 (2005), 363-392.

[10] U. Meierfrankenfeld, B. Stellmacher and G. Stroth, "Groups of local characteristic p, An overview", in Proceedings of the 2001 Durham Conference on Groups and Geometries 155-192, (2003).

[11] U. Meierfrankenfeld, B. Stellmacher and G. Stroth, "The local structure for finite groups with a large p-subgroup", Corrected title: "The local structure theorem for finite groups with a large p-subgroup, Mem. Amer. Math. Soc. 242,1147 (2016).

[12] Chr. Parker, G. Parmeggiani, B. Stellmacher, "The P!-Theorem", Journal of Algebra 263 (2003), 17-58.

[13] Chr. Parker, G. Pientka, A. Seidel, G. Stroth, "Finite groups which are almost groups of Lie type in characteristic p", submitted 2017. 
[14] Chr. Parker, P. Rowley, "Symplectic amalgams". Springer Monographs in Mathematics. Springer-Verlag London, Ltd., London, 2002.

[15] Chr. Pröseler, "Der Nicht.Charakteristik-2-Fall des lokalen Struktursatzes, Gruppen mit 2-lokalen Untergruppen vom orthogonalen Typ, Thesis Halle 2019.

[16] M. Ronan, G. Stroth, "Minimal parabolic geometries for the sporadic groups", Europ. J. Combinatorics 5, 59-91, 1984.

Chris Parker, School of Mathematics, University of Birmingham, Edgbaston, Birmingham B15 2TT, United Kingdom

E-mail address: c.w.parker@bham.ac.uk

Gernot Stroth, Institut für Mathematik, Universität Halle - WitTenberg, Theordor Lieser Str. 5, 06099 Halle, Germany

E-mail address: gernot.stroth@mathematik.uni-halle.de 